\title{
Neutrino-nucleus interactions and the determination of oscillation parameters
}

\author{
Omar Benhar ${ }^{\mathrm{a}}$, Patrick Huber ${ }^{\mathrm{b}}$, Camillo Mariani ${ }^{\mathrm{b}}$, Davide Meloni ${ }^{\mathrm{c}}$ \\ ${ }^{a}$ INFN and Department of Physics, Sapienza University, I-00185 Roma, Italy \\ ${ }^{b}$ Center for Neutrino Physics, Virginia Tech, Blacksburg, Virginia 24061, USA \\ ${ }^{c}$ Dipartimento di Matematica e Fisica, Università Roma Tre, I-00146 Roma, Italy
}

\begin{abstract}
We review the status and prospects of theoretical studies of neutrino-nucleus interactions, and discuss the influence of the treatment of nuclear effects on the determination of oscillation parameters. The models developed to describe the variety of reaction mechanisms contributing to the nuclear cross sections are analysed, with emphasis placed on their capability to explain the large body of available electron scattering data. The impact of the uncertainties associated with the description of nuclear structure and dynamics on the determination of oscillation parameters is illustrated through examples, and possible avenues towards a better understanding of the signals detected by accelerator-based experiments are outlined.
\end{abstract}

Keywords: keyword1, keyword2

PACS: code1, code2

\section{Contents}

1 Introduction

2 Comparison between electron- and neutrino-nucleus scattering

\section{The electron-nucleus cross section}

3.1 The impulse approximation . . . . . . . . . . 4

3.2 The nuclear spectral function . . . . . . . . 6

$3.3 y$-scaling and superscaling . . . . . . . . . 7

3.4 Final state interactions . . . . . . . . . . 9

3.5 Two-nucleon currents and $2 \mathrm{p} 2 \mathrm{~h}$ final states . . . 10

3.6 Collective excitations . . . . . . . . . . 12

3.7 Comparison to data . . . . . . . . . . 12

4 The flux-integrated neutrino-nucleus cross section 15

4.1 Quasielastic scattering .......... 17

4.2 Resonance production . . . . . . . . . . 17

4.3 Deep inelastic scattering . . . . . . . . . 18

\section{Interpretation of CC QE events}

6 Implementation of nuclear dynamics in Monte Carlo simulations

7 Dependence of oscillation parameters on the description of nuclear effects

7.1 The problem at rate-level . . . . . . . . . . . 24

7.2 The impact on the mixing angle measurement at $\mathrm{T} 2 \mathrm{~K} \ldots \ldots \ldots \ldots 26 . \ldots \ldots 26$

7.3 Reconstruction of neutrino energy . . . . . . 29
7.4 Detector effects impact on disappearance and appearance results . . . . . . . . 31

\section{Summary and outlook}

\section{Introduction}

Neutrino physics is entering the age of precision measurements. A number of experiments have firmly established the occurrence of neutrino oscillations and determined the corresponding squared mass differences and mixing angles [1-6]. These measurements have provided unambiguous evidence that neutrinos-assumed to be massless in the standard model of particle physics — do have non-vanishing masses. Reactor and accelerator-based experiments carried out over the past few years [7-9] reported accurate measurements of the $\theta_{13}$ mixing angle, whose value turned out to be $\sim 10 \mathrm{deg}$. The large $\theta_{13}$ mixing angle will enable future experiments-such as the Deep Underground Neutrino Experiment (DUNE) in the United States [10] - to search for leptonic CP violation in appearance mode, thus addressing one of the outstanding fundamental problems of particle physics. These searches will involve high precision determinations of the oscillation parameters, which in turn require a deep understanding of neutrino interactions with the atomic nuclei comprising the detectors. In view of the achieved and planned experimental accuracies, the treatment of nuclear effects is indeed regarded as one of the main sources of systematic uncertainty [11].

Over the past decade, it has become more and more evident that the independent particle model of nuclei-the ultimate 
implementation of which is the Relativistic Fermi Gas Model (RFGM) routinely employed in simulations of neutrino-nucleus interactions - conspicuously fails to account for the complexity of nuclear dynamics and the variety of reaction mechanisms contributing to the detected signals.

The large discrepancy between the results of Monte Carlo simulations and the double differential cross section of charged current (CC) quasielastic (QE) interactions in carbon, measured by the MiniBooNE Collaboration using a beam of average energy $\sim 0.8$ (0.7) GeV in neutrino (antineutrino) mode, is a striking manifestation of the above problem [12-14]. More recently, the analysis of the inclusive $v_{\mu}$-nucleus cross sections at beam energy in the range $2-20 \mathrm{GeV}$, measured by the MINER $v \mathrm{~A}$ Collaboration using a variety of targets, led to the striking conclusion that none of nuclear models implemented in Monte Carlo simulations appears to be capable of explaining the data [15].

A great deal of effort is currently being devoted to the development of theoretical models providing a fully quantitative description of the neutrino-nucleus cross section in the kinematical regime relevant to most ongoing and future acceleratorbased experiments, corresponding to beam energies ranging from a few hundred $\mathrm{MeV}$ to a few $\mathrm{GeV}$. In this context, a key role is played by the availability of a wealth of electron scattering data.

Electron scattering experiments have provided accurate information on the electromagnetic response of a number of nuclei. Static form factors and charge distributions have been extracted from elastic scattering data, while the measurements of inelastic cross sections have allowed for a systematic study of the dynamic response functions in a broad range of energy and momentum transfer. Finally, with the advent of the last generation of continuous beam accelerators, a number of exclusive channels have been analysed to unprecedented precision.

The large body of measured electron scattering cross sections provides an indispensable benchmark for validation of theoretical models. In fact, the ability to explain electron scattering data sholud be seen as an obvious requisite, to be met by any models of neutrino-nucleus interactions. In addition, new electron scattering experiments will be needed, to gain information on nuclei employed in neutrino detectors-most notably argon-for which the available data is scarce, or nonexisting, [16].

This review is organized as follows. In Section 2 we provide a comparative analysis between electron and neutrino-nucleus scattering, aimed at pointing out the difficulties involved in the interpretation of the flux-integrated neutrino cross sections. The theoretical description of the reaction mechanisms contributing to the electron-nucleus cross section is outlined in Section 3, where the ability of different approaches to explain the data is also illustrated. The generalisation of the formalism based on the impulse approximation to the case of neutrino interactions and the problems associated with the interpretation of the measured CC QE cross sections are discussed in Sections 4 and 5, respectively, while Section 6 is devoted to a discussion of the issues involved in the implementation of the spectral function formalism in neutrino event generators. In Section 7 we provide few examples showing how the treatment of nuclear effects affects the determination of neutrino oscillation parameters. Fi- nally, in Section 8 we summarise our assessment of both the present status and the future prospects of the field.

\section{Comparison between electron- and neutrino-nucleus scattering}

The description of electron-nucleus interactions involves a variety of non-trivial problems, arising from the complexity of both nuclear and nucleon structure and dynamics.

Figure 1 shows the typical behaviour of the double differential cross section of the inclusive process

$$
e+A \rightarrow e^{\prime}+X
$$

in which only the outgoing lepton is detected, at beam energy around $1 \mathrm{GeV}$. Here, $A$ and $X$ denote the target nucleus in its ground state and the undetected hadronic final state, respectively.

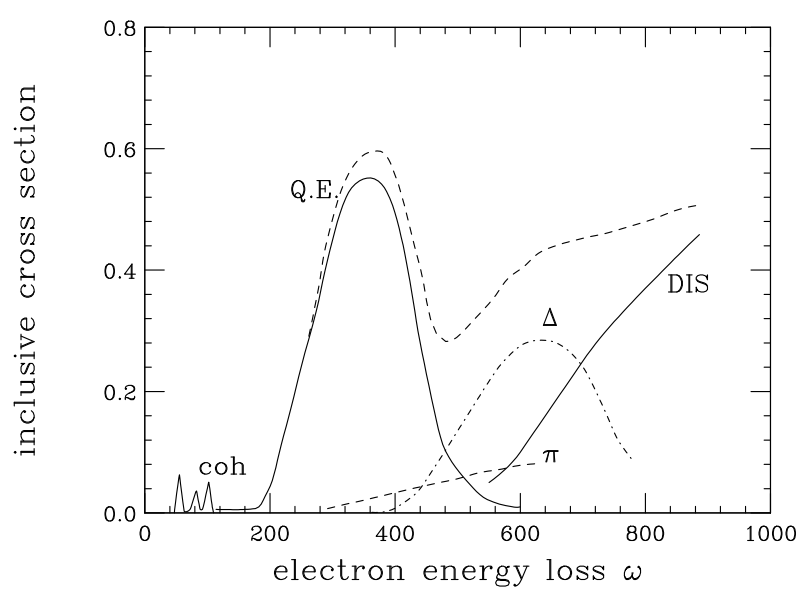

Figure 1: Schematic representation of the inclusive electron-nucleus cross section at beam energy around $1 \mathrm{GeV}$ and fixed electron scattering angle, displayed as a function of the energy transfer [17].

The data is shown for fixed electron scattering angle, $\theta_{e}$, as a function of the energy transfer $\omega=E_{e}-E_{e^{\prime}}$, the value of which is the main factor determining the dominant reaction mechanism. The bump centered at $\omega \approx \omega_{\mathrm{QE}}=Q^{2} / 2 m$, where $m$ is the nucleon mass and $Q^{2}=4 E_{e} E_{e^{\prime}} \sin ^{2}\left(\theta_{e} / 2\right)$, corresponds to single nucleon knockout, while the structure visible at larger $\omega$ reflects the onset of more complex mechanisms, such as coupling to nucleons belonging to correlated pairs or two-nucleon currents arising from meson exchange processes, excitation of nucleon resonances and deep inelastic scattering.

The analysis of electron scattering data has clearly exposed the limitations of the independent particle picture, providing the conceptual framework of the nuclear shell model [18].

Accurate measurements of the coincidence $\left(e, e^{\prime} p\right)$ cross section in the region of low to moderate missing energy and missing momentum have unambiguously demonstrated that, while the spectroscopic lines corresponding to knock 
out of nucleons occupying the shell model orbits are clearly visible in the missing energy spectra, the associated spectroscopic factors-yielding the normalization of the singlenucleon states-are considerably less than unity, regardless of the nuclear mass number $A$ (for a recent overview of $\left(e, e^{\prime} p\right)$ data, see Ref. [19]). Complementary data collected at large missing energy and missing momentum strongly suggest that this feature is a manifestation of dynamical nucleon-nucleon (NN) correlations, leading to the excitation of nucleon pairs to continuum states and to a corresponding depletion of the bound states belonging to the Fermi sea. This interpretation, strongly supported by the results of the pioneering ${ }^{3} \mathrm{He}\left(e, e^{\prime} p\right)$ experiment described in Ref. [20], has been recently confirmed by measurements carried out at Jefferson Lab [21, 22] using a carbon target.

Advanced nuclear models, developed using the formalism of many-body theory, provide an overall satisfactory description of the observed cross sections over a broad kinematical range. In particular, in the region in which QE scattering dominates the data is generally reproduced with an accuracy of few percent [23, 24] (for a recent review of electron-nucleus scattering in the QE sector see also Ref. [17]).

Nuclear Many-Body Theory (NMBT) is based on the tenet that nucleons can be treated as point like non relativistic particles, the dynamics of which are described by the Hamiltonian

$$
H=\sum_{i=1}^{A} \frac{\mathbf{p}_{i}^{2}}{2 m}+\sum_{j>i=1}^{A} v_{i j}+\sum_{k>j>i=1}^{A} V_{i j k} .
$$

In the above equation, $\mathbf{p}_{i}$ is the momentum of the $i$-th nucleon, while the potentials $v_{i j}$ and $V_{i j k}$ describe two- and three-nucleon interactions, respectively.

Phenomenological two-nucleon potentials are obtained from an accurate fit to the available data on the two-nucleon system, in both bound and scattering states, and reduce to the Yukawa one-pion-exchange potential at large distances [25]. The inclusion of the additional three-body term, $V_{i j k}$, is needed to reproduce the binding of light nuclei [26].

Recently, chiral perturbation theory, or $\chi P T$, (for a review, see Refs. [27, 28]) has been also employed to obtain a theoretically sound and consistent model of both two- and threenucleon interactions, constrained by the symmetries of the fundamental theory of strong interactions. This approach, originally proposed by Weinberg, exploits the Goldstone boson nature of the pion [29], implying that the interactions of low energy pions are weak, and can be treated in perturbation theory.

The nuclear electromagnetic current, $J^{\mu} \equiv\left(J^{0}, \mathbf{J}\right)$, is related to the Hamiltonian (2) through the continuity equation [30]

$$
\nabla \cdot \mathbf{J}+i\left[H, J^{0}\right]=0 .
$$

Because the NN potential $v_{i j}$ does not commute with the charge operator $J^{0}$, the above equation implies that $J^{\mu}$ comprises twonucleon contributions, arising from meson exchange processes. Therefore, it can be conveniently written in the form

$$
J^{\mu}=J_{1}^{\mu}+J_{2}^{\mu}=\sum_{i} j_{i}^{\mu}+\sum_{\mathrm{j}>\mathrm{i}} j_{i j}^{\mu} .
$$

The main difficulty associated with the extension of the theoretical approaches developed for electron-nucleus scattering to the case of neutrino scattering arises from the fact that, since neutrino beams are always produced as secondary decay products, their energy is not sharply defined, but broadly distributed according to a flux $\Phi$.

Consider, for example, charged-current neutrino interactions. In this instance, detection of the energy of the outgoing lepton, $T_{\ell}$, does not provide the information on the energy transfer, $\omega$, and different reaction mechanisms contribute to the double differential cross section measured at fixed $T_{\ell}$ and lepton scattering angle, $\theta_{\ell}$.

This feature is clearly illustrated in Fig. 2, showing the inclusive electron-carbon cross sections at $\theta_{e}=37 \mathrm{deg}$ and beam energies ranging between 0.730 and $1.501 \mathrm{GeV}$, as a function of the energy of the outgoing electron $[31,32]$. It clearly appears that the highlighted electron energy bin $\left(550<T_{e^{\prime}}<\right.$ $650 \mathrm{MeV}$ ), corresponding to QE kinematics at $E_{e}=730 \mathrm{MeV}$, picks up contributions from scattering processes taking place at different beam energies, in which reaction mechanisms other than single nucleon knockout dominate.

To gauge the role played by different contributions in a typical neutrino experiment, let us assume that the electron beam energy be distributed according to the MiniBooNE neutrino flux, displayed in Fig. 3. It turns out that the fluxes corresponding to energies $E_{v}=730$ and $961 \mathrm{MeV}$ are within $\sim 20 \%$ of one another. Hence, if we were to average the electron-carbon data of Fig. 2 with the flux of Fig. 3, the cross sections corresponding to beam energies 730 and $961 \mathrm{MeV}$ would contribute to the measured cross section in the highlighted bin with about the same weight.

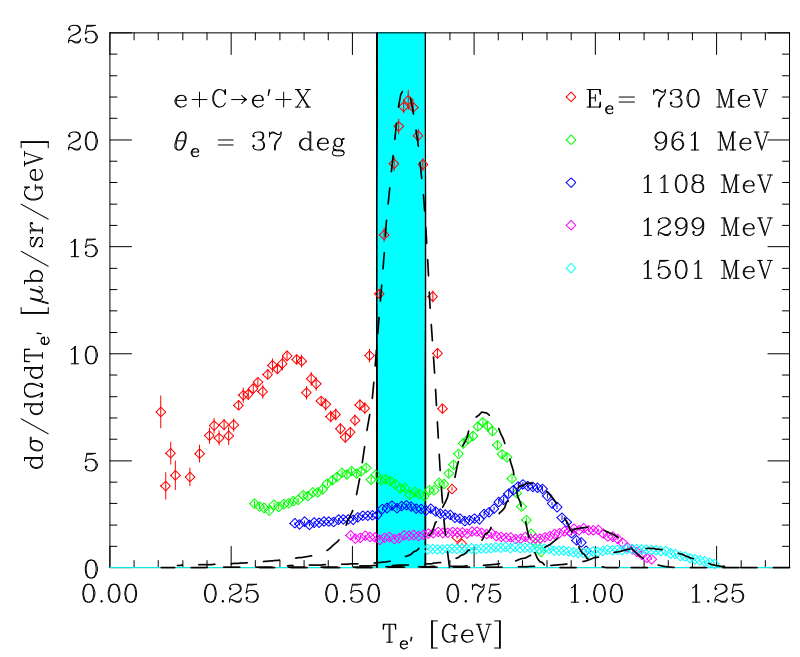

Figure 2: Inclusive electron-carbon cross sections at $\theta_{e}=37 \mathrm{deg}$ and beam energies ranging between 0.730 and $1.501 \mathrm{GeV}$ [31,32], plotted as a function of the energy of the outgoing electron. The dashed lines represent the results of theoretical calculations, carried out within the spectral function approach (see Section 3.1) taking into account QE scattering only [33].

The above discussion implies that the understanding of the flux-averaged neutrino cross section requires the development of theoretical models providing a consistent treatment of all re- 


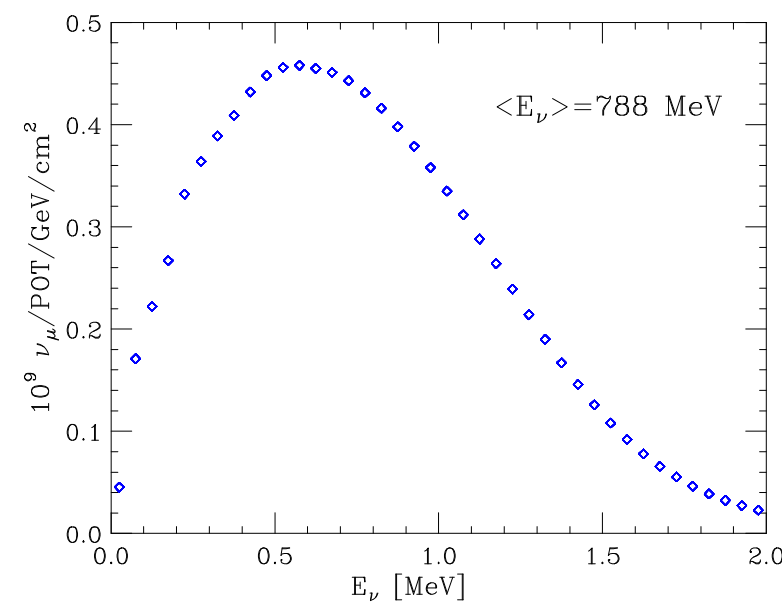

Figure 3: Energy dependence of the MiniBooNE neutrino flux [13].

action mechanisms active in the broad kinematical range corresponding to the relevant neutrino energies.

\section{The electron-nucleus cross section}

The differential cross section of process (1), in which an electron of initial four-momentum $k_{e} \equiv\left(E_{e}, \mathbf{k}_{e}\right)$ scatters off a nuclear target to a state of four-momentum $k_{e}^{\prime} \equiv\left(E_{e^{\prime}}, \mathbf{k}_{e^{\prime}}\right)$, the target final state being undetected, can be written in Born approximation as (see, e.g., Ref. [34])

$$
\frac{d^{2} \sigma}{d \Omega_{e^{\prime}} d E_{e^{\prime}}}=\frac{\alpha^{2}}{Q^{4}} \frac{E_{e^{\prime}}}{E_{e}} L_{\lambda \mu} W^{\lambda \mu},
$$

where $\alpha=1 / 137$ is the fine structure constant, $d \Omega_{e^{\prime}}$ is the differential solid angle in the direction specified by $\mathbf{k}_{e^{\prime}}, Q^{2}=-q^{2}$ and $q=k_{e}-k_{e^{\prime}} \equiv(\omega, \mathbf{q})$ is the four momentum transfer.

The tensor $L^{\lambda \mu}$, that neglecting the electron mass reduces to

$$
L^{\lambda \mu}=2\left[k_{e}^{\lambda} k_{e^{\prime}}^{\mu}+k_{e}^{\mu} k_{e^{\prime}}^{\lambda}-g^{\lambda \mu}\left(k_{e} k_{e^{\prime}}\right)\right],
$$

where $g^{\lambda \mu} \equiv \operatorname{diag}(1,-1,-1,-1)$ and $\left(k_{e} k_{e^{\prime}}\right)=E_{e} E_{e^{\prime}}-\mathbf{k}_{e} \cdot \mathbf{k}_{e^{\prime}}$, is fully specified by the measured electron kinematical variables.

All information on target structure is contained in the tensor $W^{\lambda \mu}$, the definition of which involves the initial and final nuclear states $|0\rangle$ and $|X\rangle$, carrying four-momenta $P_{0}$ and $P_{X}$, as well as the nuclear current operator of Eq. (4). It can be cast in the form

$$
W^{\lambda \mu}=\sum_{X}\left\langle 0\left|J^{\lambda}\right| X\right\rangle\left\langle X\left|J^{\mu}\right| 0\right\rangle \delta^{(4)}\left(P_{0}+q-P_{X}\right),
$$

where the sum includes all hadronic final states.

The most general expression of the target tensor of Eq. (7), fulfilling the requirements of Lorentz covariance, conservation of parity and gauge invariance, can be written in terms of two structure functions $W_{1}$ and $W_{2}$ as

$$
\begin{aligned}
W^{\lambda \mu} & =W_{1}\left(-g^{\lambda \mu}+\frac{q^{\lambda} q^{\mu}}{q^{2}}\right) \\
& +\frac{W_{2}}{M_{A}^{2}}\left(P_{0}^{\lambda}-\frac{\left(P_{0} q\right)}{q^{2}} q^{\lambda}\right)\left(P_{0}^{\mu}-\frac{\left(P_{0} q\right)}{q^{2}} q^{\mu}\right),
\end{aligned}
$$

where $M_{A}$ is the target mass and the structure functions depend on the two scalar quantities $Q^{2}$ and $\left(P_{0} q\right)$. In the target rest frame $\left(P_{0} q\right)=M_{A} \omega$, and $W_{1}$ and $W_{2}$ become functions of the measured momentum and energy transfer, $|\mathbf{q}|$ and $\omega$.

Substitution of Eq. (8) into Eq. (5) leads to

$$
\frac{d^{2} \sigma}{d \Omega_{e^{\prime}} d E_{e^{\prime}}}=\left(\frac{d \sigma}{d \Omega_{e^{\prime}}}\right)_{\mathrm{M}}\left[W_{2}(|\mathbf{q}|, \omega)+2 W_{1}(|\mathbf{q}|, \omega) \tan ^{2} \frac{\theta_{e}}{2}\right],
$$

where $\left(d \sigma / d \Omega_{e^{\prime}}\right)_{\mathrm{M}}=\alpha^{2} \cos ^{2}\left(\theta_{e} / 2\right) / 4 E_{e}^{2} \sin ^{4}\left(\theta_{e} / 2\right)$ is the Mott cross section.

The right-hand side of Eq. (9) can be conveniently rewritten in terms of the contributions arising from scattering processes involving longitudinally (L) and transversely $(\mathrm{T})$ polarized virtual photons. The resulting expression is

$$
\begin{aligned}
& \frac{d^{2} \sigma}{d \Omega_{e^{\prime}} d E_{e^{\prime}}}=\left(\frac{d \sigma}{d \Omega_{e^{\prime}}}\right)_{\mathrm{M}} {\left[\left(\frac{Q^{2}}{|\mathbf{q}|^{2}}\right)^{2} R_{L}(|\mathbf{q}|, \omega)\right.} \\
&\left.+\left(\frac{1}{2} \frac{Q^{2}}{|\mathbf{q}|^{2}}+\tan ^{2} \frac{\theta}{2}\right) R_{T}(|\mathbf{q}|, \omega)\right],
\end{aligned}
$$

where the longitudinal and transverse structure functions, $R_{L}$ and $R_{T}$, are trivially related to $W_{1}$ and $W_{2}$ through

$$
R_{T}=2 W_{1}
$$

and

$$
\left(\frac{Q^{2}}{|\mathbf{q}|^{2}}\right)^{2} R_{L}=W_{2}-\frac{Q^{2}}{|\mathbf{q}|^{2}} W_{1} .
$$

The initial state of the target nucleus appearing in Eq. (7) can be safely treated using the non relativistic approximation, regardless of the kinematical regime. At large momentum transfer, however, this approximation can not be used to describe either the nuclear final state, comprising at least one particle carrying momentum $\sim \mathbf{q}$, or the current operator, which depends explicitly on momentum transfer.

At low and moderate momentum transfer, typically $|\mathbf{q}|<500 \mathrm{MeV}$, accurate NMBT calculations of the tensor $W^{\lambda \mu}$ of Eq. (7) have been carried out for the few-nucleon systems, using nuclear wave functions derived from the Hamiltonian of Eq. (2) to describe the initial and final states and expanding the current operator in powers of $|\mathbf{q}| / m$ [35]. Valuable information have been also obtained exploiting integral transform techniques [36-40].

On the other hand, additional assumptions are unavoidably required for the treatment of nuclear interactions in the region of large momentum transfer, the understanding of which is relevant to accelerator based neutrino experiment. To importance of relativistic effects can be easily gauged considering that the mean momentum transfer of CC QE events obtained by averaging over the MiniBooNE [13] and Minerva [15] neutrino fluxes turn out to be $\sim 640$ and $\sim 880 \mathrm{MeV}$, respectively.

\subsection{The impulse approximation}

The Impulse Approximation (IA) scheme, extensively employed to analyze electron-nucleus scattering data in the region in which the non relativistic approximation breaks down [17], 
is based on the premise that at momentum transfer $\mathbf{q}$ such that $|\mathbf{q}|^{-1} \ll d / \pi, d$ being the average distance between nucleons in the target nucleus, the nuclear scattering process reduces to an incoherent sum of collisions involving individual nucleons-as schematically illustrated by the diagram of Fig. 4- the remaining $\mathrm{A}-1$ particles acting as spectators. Moreover, as a first approximation, final state interactions (FSI) between the outgoing hadrons and the spectator nucleons are assumed to be negligible. Within this picture, the relativistic particles in the final state are completely decoupled from the recoiling nucleus, and the description of their motion becomes a trivial kinematical problem ${ }^{1}$.

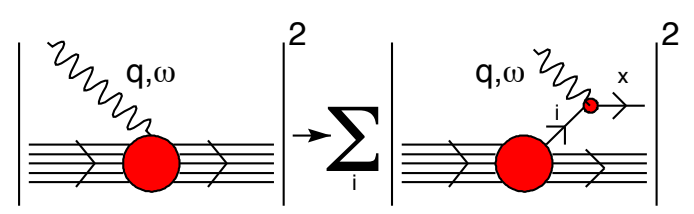

Figure 4: Schematic representation of the IA regime, in which the nuclear cross section is replaced by the incoherent sum of cross sections describing scattering off individual nucleons, with the recoiling $(\mathrm{A}-1)$-nucleon system acting as a spectator.

Under the assumptions underlying the IA, the nuclear current of Eq.(4) simplifies to a sum of one-body terms, while the final state factorizes into the product of the hadronic state $x$, produced at the interaction vertex with momentum $\mathbf{p}_{x}$, and the state describing the recoiling $(A-1)$-nucleon system, carrying momentum $\mathbf{p}_{\mathrm{R}}{ }^{2}$. As a consequence, in Eq. (7) we can replace

$$
|X\rangle \longrightarrow\left|x, \mathbf{p}_{x}\right\rangle \otimes\left|\mathrm{R}, \mathbf{p}_{\mathrm{R}}\right\rangle,
$$

implying

$$
\begin{aligned}
\sum_{X}|X\rangle\langle X| \rightarrow & \sum_{x} \int d^{3} p_{x}\left|x, \mathbf{p}_{x}\right\rangle\left\langle\mathbf{p}_{x}, x\right| \\
& \times \sum_{\mathrm{R}} \int d^{3} p_{\mathrm{R}}\left|\mathrm{R}, \mathbf{p}_{\mathrm{R}}\right\rangle\left\langle\mathbf{p}_{\mathrm{R}}, \mathrm{R}\right|,
\end{aligned}
$$

where the integrations include a sum over all discrete quantum numbers. The resulting nuclear transition matrix elements take the form

$$
\left\langle 0\left|J^{\mu}\right| X\right\rangle=\sqrt{\frac{m}{E_{p_{\mathrm{R}}}}} M_{\mathrm{R}}\left(\mathbf{p}_{\mathrm{R}}\right) \sum_{i}\left\langle-\mathbf{p}_{\mathrm{R}}, N\left|j_{i}^{\mu}\right| x, \mathbf{p}_{x}\right\rangle,
$$

where the state $|N, \mathbf{k}\rangle$ decribes a non interacting nucleon $(N=$ $p, n$ denotes protons and neutrons) carrying momentum $\mathbf{k}$, and

$$
M_{\mathrm{R}}\left(\mathbf{p}_{\mathrm{R}}\right)=\left\langle0 \left|\left\{\left|\mathrm{R}, \mathbf{p}_{\mathrm{R}}\right\rangle \otimes\left|N,-\mathbf{p}_{\mathrm{R}}\right\rangle\right\},\right.\right.
$$

\footnotetext{
${ }^{1}$ In the literature the approximation scheme in which FSI are neglected is sometimes referred to as Plane Wave Impulse Approximation (PWIA).

${ }^{2}$ Note that the discussion of this Section is not restricted to the QE sector. To the extent to which a description of the elementary interaction process is available, it can be readily applied to resonance production and deep-inelastic scattering.
}

with $E_{p_{\mathrm{R}}}=\sqrt{\left|\mathbf{p}_{\mathrm{R}}\right|^{2}+m^{2}}$.

Being independent of $\mathbf{q}$, the nuclear amplitude $M_{\mathrm{R}}\left(\mathbf{p}_{\mathrm{R}}\right)$ can be safely obtained from NMBT. On the other hand, the calculation of the matrix element of the current operator between free nucleon states can be carried out for all values of the momentum transfer without employing any approximations, assumin that the nucleon form factors are precisely known.

Substituting Eq.(15) into Eq. (7) and using Eq.(14), one can rewrite the target tensor in the concise and transparent form

$$
W^{\lambda \mu}=\int d^{3} k d E \frac{M}{E_{k}} P(\mathbf{k}, E)\left[Z \mathcal{W}_{p}^{\lambda \mu}+(A-Z) \mathcal{W}_{n}^{\lambda \mu}\right],
$$

where $Z$ is the target charge, and the spectral function $P(\mathbf{k}, E)$, yielding the probability of removing a nucleon with momentum $\mathbf{k}$ from the target ground state leaving the residual system with excitation energy $E$, is defined ss $^{3}[41,42]$

$$
P(\mathbf{k}, E)=\sum_{\mathrm{R}}\left|M_{\mathrm{R}}(\mathbf{k})\right|^{2} \delta\left(E+M_{A}-m-E_{\mathrm{R}}\right),
$$

$E_{\mathrm{R}}$ being the energy of the state $|\mathrm{R}, \mathbf{k}\rangle$.

The tensor

$$
\begin{aligned}
\mathcal{W}_{N}^{\lambda \mu}=\sum_{x} & \int d^{3} p_{x}\left\langle\mathbf{k}, N\left|j_{N}^{\lambda}\right| x, \mathbf{p}_{x}\right\rangle\left\langle\mathbf{p}_{x}, x\left|j_{N}^{\mu}\right| N, \mathbf{k}\right\rangle \\
& \times \delta\left(\tilde{\omega}+E_{k}-E_{x}\right) \delta^{(3)}\left(\mathbf{k}+\mathbf{q}-\mathbf{p}_{x}\right),
\end{aligned}
$$

where $E_{x}$ is the energy of the hadronic final state carrying momentum $\mathbf{p}_{x}=\mathbf{k}+\mathbf{q}$, describes the electromagnetic interactions of a nucleon with four momentum $k \equiv\left(E_{k}, \mathbf{k}\right)$ in free space. Note, however, that the four momentum transfer $q$ is replaced by $\tilde{q} \equiv(\tilde{\omega}, \mathbf{q})$, with

$$
\tilde{\omega}=E_{x}-E_{k}=\omega+m-E-E_{k} .
$$

The substitution $\omega \rightarrow \tilde{\omega}$ is needed to take into account the fact that a fraction $\delta \omega$ of the energy transfer to the target goes into excitation energy of the spectator system. Equation (19) shows that the elementary scattering process is described as if it took place in free space with energy transfer $\tilde{\omega}=\omega-\delta \omega$.

It has to be pointed out that, while sensible on physics grounds, the use of $\tilde{q}$ in the nucleon tensor (19) poses a non trivial conceptual problem, in that it leads to a violation of current conservation. This problem is inherent in the IA scheme, which does not allow energy and current to be simultaneously conserved. A very effective prescription to restore gauge invariance, extensively employed in the analysis of $\left(e, e^{\prime} p\right)$ experiments, is based on the use of off-shell extrapolations of the electron-nucleon cross section, referred to as $c c 1$ and $c c 2$, developed by de Forest in the early 80s [43]. Note, however, that in QE kinematics, because the struck nucleon is nearly on the mass shell, the effect of using de Forest's prescription turns out to be quite small.

\footnotetext{
${ }^{3}$ In deriving Eq. (17) we have made the assumption, largely justified in isoscalar nuclei, that the proton and neutron spectral functions be identical.
} 
Collecting the above results, the nuclear cross section can be finally written in the form

$$
\frac{d^{2} \sigma_{I A}}{d \Omega_{e^{\prime}} d E_{e^{\prime}}}=\int d^{3} k d E P(\mathbf{k}, E)\left[Z \frac{d^{2} \sigma_{e p}}{d \Omega_{e^{\prime}} d E_{e^{\prime}}}+N \frac{d^{2} \sigma_{e n}}{d \Omega_{e^{\prime}} d E_{e^{\prime}}}\right],
$$

with

$$
\frac{d^{2} \sigma_{\mathrm{eN}}}{d \Omega_{e^{\prime}} d E_{e^{\prime}}}=\frac{\alpha^{2}}{Q^{4}} \frac{E_{e^{\prime}}}{E_{e}} L_{\lambda \mu} W_{N}^{\lambda \mu} .
$$

Equation (21), first derived by the authors of Ref. [44], has been widely used in the course of four decades. It shows that, within the IA scheme, the electron-nucleus cross section can be obtained folding the cross sections of the processes involving individual nucleons - which can be, at least in principle, measured using proton and deuteron targets-with the energy and momentum distribution of the participating nucleon, described by the spectral function.

Note that in Eq.(21) the effect of Pauli blocking on the phase space available to the struck nucleon in the final state-which becomes vanishingly small in the limit of large momentum transfer-is disregarded altogether. A simple and reasonable procedure to take it into account is based on the replacement

$$
P(\mathbf{k}, E) \rightarrow P(\mathbf{k}, E) \theta\left(|\mathbf{k}+\mathbf{q}|-\bar{k}_{F}\right),
$$

where $\theta(x)$ is the Heaviside step function and $\bar{k}_{F}$ is an average nuclear Fermi momentum, derived within the local Fermi gas model [23, 24].

\subsection{The nuclear spectral function}

Within the mean field approximation underlying the independent particle model (IPM) of the nucleus, the sum over the sates of the residual (A - 1)-nucleon system appearing in Eq. (18) is restricted to bound one-hole states. The corresponding spectral function can be written in the form

$$
P_{\mathrm{MF}}(\mathbf{k}, E)=\sum_{\alpha \in\{F\}}\left|\phi_{\alpha}(\mathbf{k})\right|^{2} \delta\left(E-\epsilon_{\alpha}\right),
$$

where the sum includes all single particle states belonging to the Fermi sea $\{F\}$, labeled by the index $\alpha$, with $\phi_{\alpha}(\mathbf{k})$ and $\epsilon_{\alpha}$ being the corresponding momentum-space wave function and energy, respectively. Note that $\left|\phi_{\alpha}(\mathbf{k})\right|^{2}$ yields the probability of finding a nucleon with momentum $\mathbf{k}$ in the state $\alpha$.

The mean field approximation provides a fairly good description of the spectral functions at $|\mathbf{k}| \lesssim 250 \mathrm{MeV}$, and $E$ lower than the energies required to remove a nucleon from the shell model states.

A wealth of experimental information on the nuclear spectral functions in the kinematical regime in which mean field dynamics is dominant has been extracted from the cross sections of the $\left(e, e^{\prime} p\right)$ process, measured using a variety of targets (for extensive reviews, see Refs. [45, 46]).

Within the RFGM, the single-particle states, labeled by the momentum $\mathbf{k}$, are occupied with unit probability for $|\mathbf{k}|<k_{F}$, $k_{F}$ being the Fermi momentum, while all levels corresponding to $|\mathbf{k}|>k_{F}$ are empty. As a consequence, the spectral function reduces to

$$
P_{\mathrm{FG}}(\mathbf{k}, E)=\frac{3}{4 \pi k_{F}^{3}} \theta\left(k_{F}-|\mathbf{k}|\right) \delta\left(E-E_{k}-\bar{\epsilon}\right),
$$

where $E_{k}$ is the energy of a non interacting nucleon of momentum $\mathbf{k}$, and $\bar{\epsilon}$ is an average binding energy.

The values of the two parameters of the RFGM, $k_{F}$ and $\bar{\epsilon}$, are inferred from the width and position of the peak exhibited by the measured electron-nucleus cross sections in the QE channel, respectively [47].

It is very important to realise that, when using a realistic nuclear model, in which the effects of NN correlations are taken into account, more complex states, with at least one of the spectator nucleons excited to the continuum, give non vanishing contributions to the spectral function. Accurate calculations carried out for a variety of nuclear systems suggest that these contributions, arising form short-range dynamics, are largely unaffected by surface and shell effects, and are therefore nearly independent of $A$ for $A>2$. This feature is illustrated in Fig. 5, showing the $A$-dependence of the momentum distribution, defined as

$$
n(\mathbf{k})=\int d E P(\mathbf{k}, E)
$$

It clearly appears that in the region of $|\mathbf{k}| \gtrsim 1.5 \mathrm{fm}^{-1}$, or $|\mathbf{k}| \gtrsim 300 \mathrm{MeV}$, in which short-range interactions dominate, the curves corresponding to systems other than the deuteron come very close to one another. Note that in this region the momentum distributions predicted by the IPM are order of magnitudes lower than those displayed in Fig. 5, or vanish altogether.

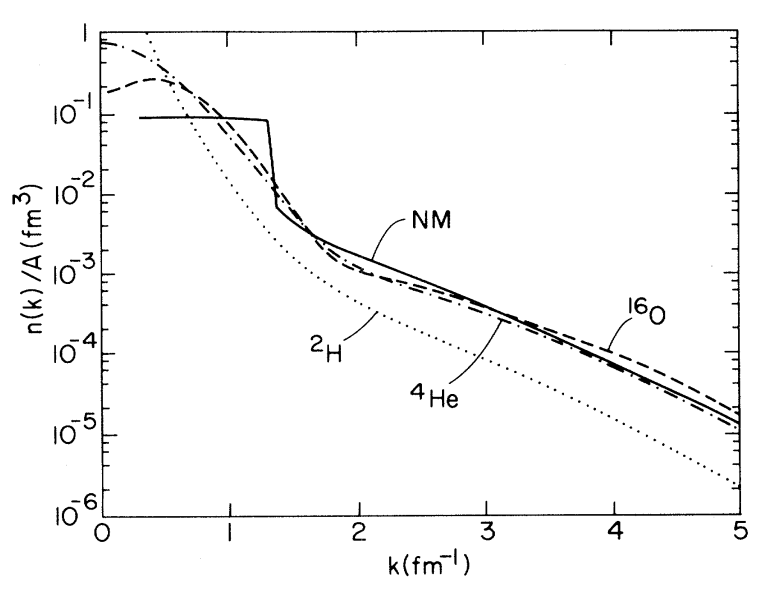

Figure 5: Momentum distribution per nucleon in ${ }^{2} \mathrm{H},{ }^{4} \mathrm{He},{ }^{16} \mathrm{O}$ and uniform isospin-symmetric nuclear matter (NM), obtained from NMBT using realistic nuclear Hamiltonians [18, 48].

Highly accurate theoretical calculations of the spectral function can be carried out for uniform nuclear matter, exploiting the simplifications granted by translation invariance [41]. The results of these calculations have been combined with the information obtained from $\left(e, e^{\prime} p\right)$ experiments to obtain spectral functions of a variety of nuclei within the Local Density Approximation (LDA) [23, 42]. 
Being trivially related to the two-point Green's function, the spectral function can be split into two parts exhibiting distinct analytical structures, as prescribed by the Källén-Lehman representation [34]. The resulting decomposition, which combined with the universality of correlation effects provides the basis of the LDA scheme, turns out to be [49]

$$
P(\mathbf{k}, E)=P_{\mathrm{MF}}(\mathbf{k}, E)+P_{\text {corr }}(\mathbf{k}, E) .
$$

The first term in the right-hand side of the above equation, describing the contribution arising from the nuclear mean field, exhibits a collection of peaks corresponding to the energies of the shell-model states belonging to the Fermi sea, while correlations provide a smooth background extending to large energy and momentum.

The mean field term is usually written in the factorized form [compare to Eq. (24)]

$$
P_{\mathrm{MF}}(\mathbf{k}, E)=\sum_{\alpha \in\{F\}} Z_{\alpha}\left|\phi_{\alpha}(\mathbf{k})\right|^{2} F_{\alpha}\left(E-\epsilon_{\alpha}\right) .
$$

The spectroscopic factors $Z_{\alpha}<1$ and the functions $F_{\alpha}\left(E-\epsilon_{\alpha}\right)$, accounting for the finite width of the $\alpha$-th shell-model state, describe the effects of residual interactions not included in the mean-field picture. In the absence of these interactions, $Z_{\alpha} \rightarrow 1, F_{\alpha}\left(E-\epsilon_{\alpha}\right) \rightarrow \delta\left(E-\epsilon_{\alpha}\right)$, and Eq. (24) is recovered.

Within LDA, the correlation contribution is obtained from

$$
P_{\mathrm{corr}}^{\mathrm{LDA}}(\mathbf{k}, E)=\int d^{3} r \varrho_{A}(\mathbf{r}) P_{\mathrm{corr}}^{\mathrm{NM}}\left(\mathbf{k}, E ; \varrho=\varrho_{A}(\mathbf{r})\right),
$$

where $\varrho_{A}(\mathbf{r})$ is the nuclear density distribution and $P_{\text {corr }}^{\mathrm{NM}}(\mathbf{k}, E ; \varrho)$ is the continuum part of the spectral function of nuclear matter at uniform density $\varrho$. Note that the spectroscopic factors $Z_{\alpha}$ are constrained by the requirement

$$
\int d^{3} k d E P_{L D A}(\mathbf{k}, E)=1
$$

with

$$
P_{\mathrm{LDA}}(\mathbf{k}, E)=P_{\mathrm{MF}}(\mathbf{k}, E)+P_{\mathrm{corr}}^{\mathrm{LDA}}(\mathbf{k}, E) .
$$

Typically, the mean-field contribution accounts for $\sim 80 \%$ of the normalisation. The $\sim 20 \%$ correlation contribution, residing at large $|\mathbf{k}|$ and $E$, has been recently measured at Jefferson Lab using a carbon target. The results of this analysis are consistent with the data at low missing energy and missing momentum, as well as with the results of theoretical calculations carried out within NMBT [21, 22].

The oxygen spectral function of Ref. [23], obtained within the LDA approximation using the results of nuclear matter calculations performed in Correlated Basis Function (CBF) perturbation theory, is shown in Fig. 6. The peaks corresponding to the shell model states are clearly visible, as is the broad background contribution arising from removal of a nucleon belonging to a correlated pair.

A very important consequence of the presence of the continuum component of the spectral function is that Eq. (21), in

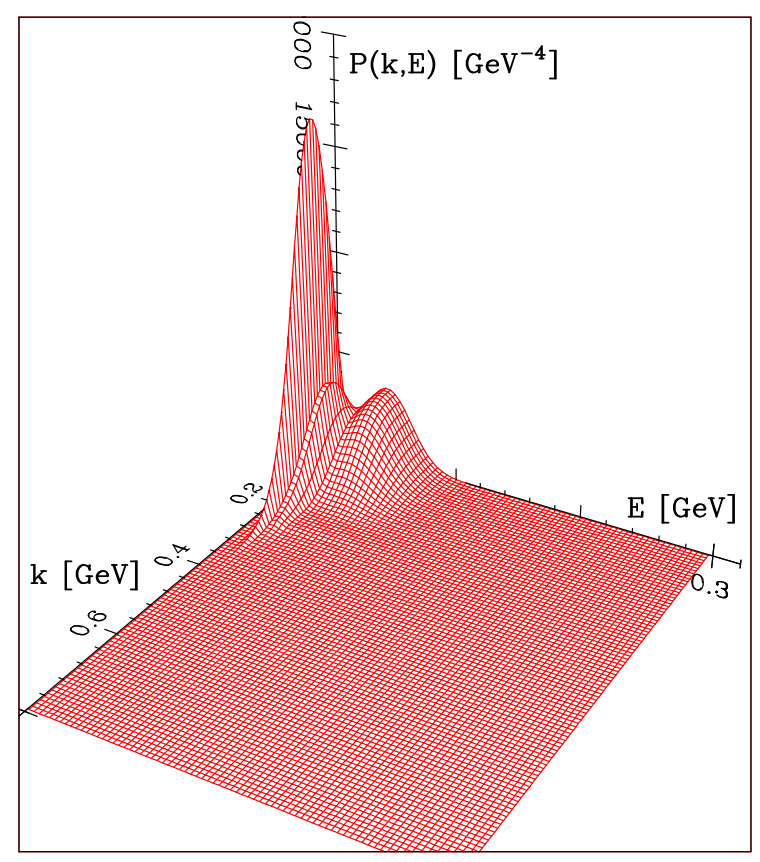

Figure 6: Three-dimensional plot of the oxygen spectral function of Ref. [23], obtained from the LDA approach.

addition to single-nucleon knock out processes-in which the target nucleus is left in a bound one-particle-one-hole (1p1h) state-also describes interactions leading to the excitation of two-particle-two-hole $(2 \mathrm{p} 2 \mathrm{~h})$ final states. As an example, Fig. 7, shows the inclusive electron-carbon cross section in the QE channel, at beam energy $E_{e}=961 \mathrm{MeV}$ and scattering angle $\theta_{e}=37.5 \mathrm{deg}$. The dot-dash and dashed lines, obtained using Eq. (21) and the carbon spectral function of Ref. [42], correspond to the cross sections of processes involving $1 \mathrm{p} 1 \mathrm{~h}$ and $2 \mathrm{p} 2 \mathrm{~h}$ final states, respectively, while the solid line shows the results of the full calculation.

Note that the $2 \mathrm{p} 2 \mathrm{~h}$ contribution displayed in Fig. 7 originates from initial state dynamics only, and would be vanishing in the absence of ground-state correlations. The appearance of additional $2 \mathrm{p} 2 \mathrm{~h}$ contributions arising form FSI will be discussed in Section 3.4.

\section{3. $y$-scaling and superscaling}

Scaling in the variable $y$ in the QE sector is a manifestation of the dominance of single nucleon knock out, which allows to write the equation expressing conservation of energy in a simplified form.

As a consequence, in the limit of large momentum transfer the function

$$
F(|\mathbf{q}|, \omega)=\frac{1}{Z \sigma_{e p}+(A-Z) \sigma_{e n}}\left(\frac{d \omega}{d k_{\|}}\right)_{k=k_{\min }} \frac{d^{2} \sigma}{d \Omega_{e^{\prime}} d E_{e^{\prime}}},
$$

which in general depends on both $|\mathbf{q}|$ and $\omega$, becomes a function of the single variable $y=y(|\mathbf{q}|, \omega)$, defined by the equation [51, 


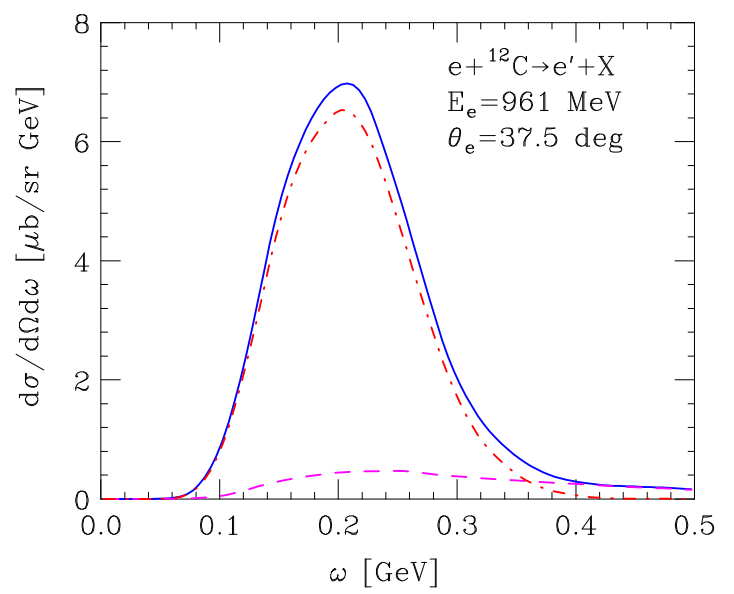

Figure 7: Inclusive electron-carbon cross section at $E_{e}=961 \mathrm{MeV}$ and $\theta_{e}=37.5 \mathrm{deg}$, plotted as a function of the energy loss $\omega$. The solid line corresponds to the result of the full IA calculation, while the dot-dash and dashed lines have been obtained, respectively, including only $1 \mathrm{p} 1 \mathrm{~h}$ and $2 \mathrm{p} 2 \mathrm{~h}$ final states [50].

52]

$$
\omega+M_{A}=\sqrt{(y+|\mathbf{q}|)^{2}+m^{2}}+\sqrt{y^{2}+\left(M_{A}-m+E_{\min }\right)^{2}} .
$$

In Eq. (32), $k_{\|}=|\mathbf{k} \cdot \mathbf{q}|$, and $k_{\min } \equiv\left(E_{\min }, \mathbf{k}_{\min }\right)$, with $E_{\min }$ and $\left|\mathbf{k}_{\text {min }}\right|$ being the lowest values of the energy and momentum of the struck nucleon allowed by the kinematical setup. The quantities $\sigma_{e p}$ and $\sigma_{e n}$ are the elementary electron-proton and electron-neutron cross sections in the QE channel, evaluated at $k=k_{\min }$ and stripped of the energy conserving $\delta$-function.

The onset of $y$-scaling is clearly illustrated in Fig. 8, showing the iron data collected at Jefferson Lab by the E89-008 Collaboration [53]. The values of $|\mathbf{q}|$ listed in the figures correspond to $\mathrm{QE}$ kinematics, i.e. to $\omega=\omega_{\mathrm{QE}}=Q^{2} / 2 \mathrm{~m}$. It clearly appears that the inclusive cross sections measured over a broad range of momentum transfer, displayed in panel (A), collapse to the scaling function $F(y)$ of panel $(B)$ in the region $y<0$, corresponding to energy transfer $\omega<\omega_{\mathrm{QE}}$. On the other hand, large scaling violations, arising from the presence of reaction mechanisms other than single nucleon knock out, are visible at $y>0$, or $\omega>\omega_{\mathrm{QE}}$ (see Fig. 1).

As pointed out above, scaling in the variable $y$, also referred to as scaling of first kind, reflects the $|\mathbf{q}|$-independence of the nuclear response at large momentum transfer. A more general form of scaling, dubbed scaling of second kind, allows to eliminate the dependence of the scaling function $F(y)$ on the nuclear target [54]. In Fig. 9, the secon-kind scaling functions of nuclei with mass number $12 \leq \mathrm{A} \leq 197$ are shown as a function of the variable $\psi^{\prime}=y / k_{F}$, where $y$ is defined by Eq. (33) and $k_{F}$ is the nuclear Fermi momentum. The functions $f\left(\psi^{\prime}\right)$ have been obtained from the data of Ref. [55] at beam energy $E_{e}=3.6 \mathrm{GeV}$ and electron scattering angle $\theta_{e}=16 \mathrm{deg}$, corresponding to $|\mathbf{q}| \sim 1 \mathrm{GeV}$. Simultaneous occurrence of scaling of first and second kind is referred to as superscaling.

The better quality of scaling of second kind, clearly appearing from a comparison between Fig. 8 (B) and Fig. 9 can be
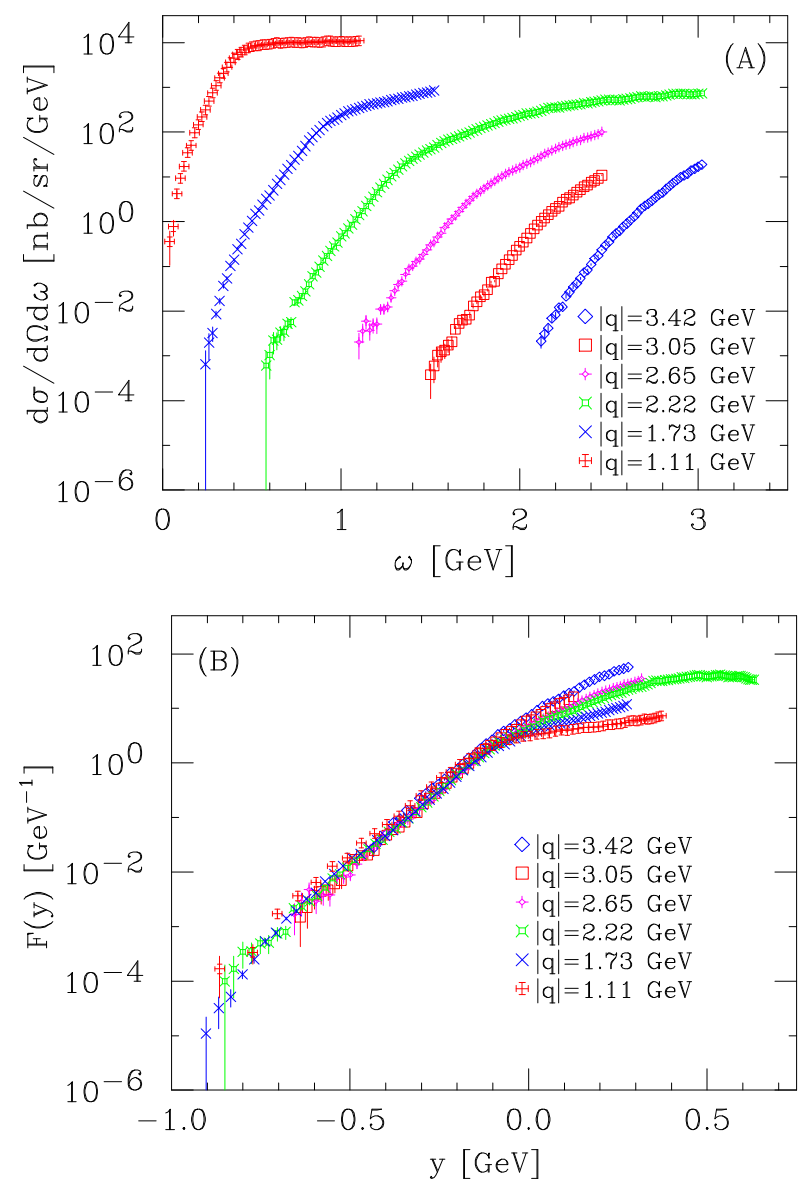

Figure 8: Panel (A): inclusive electron-iron cross sections measured at Jefferson Lab [53]. Panel (B): $y$-scaling function, defined by Eqs. (32) and (33), obtained from the data shown in panel (A). The values of $|\mathbf{q}|$ correspond to $\mathrm{QE}$ kinematics.

easily explained considering that-unlike those of Fig. 8-the data of Fig. 9 correspond to a fixed kinematical setup. Therefore, scaling in the variable $\psi^{\prime}$ merely reflects the universality of the high momentum tail of the nuclear momentum distributions, illustrated in Fig. 5.

The superscaling hypotesis has been recently extended to the kinematical region corresponding to $\omega>\omega_{\mathrm{QE}}$, in which singlenucleon knockout predominantly leads to sresonance production. Within this approach, the contribution of inelastic channels is obtained by subtracting from the data the effective QE cross section resulting from the superscaling analysis [56].

Besides allowing to identify the dominant reaction mechanism, the occurrence of superscaling can be exploited to predict the nuclear cross section for kinematical regions and targets not covered by the available data. However, the inclusion of contributions arising from mechanisms leading to large scaling violations, such as meson-exchange currents (MEC), necessarily requires the use of specific models. The universal scaling function extracted from electron scattering data has been extensively used to obtain both charged- and neutral-current neutrino-nucleus cross sections $[57,58]$. 


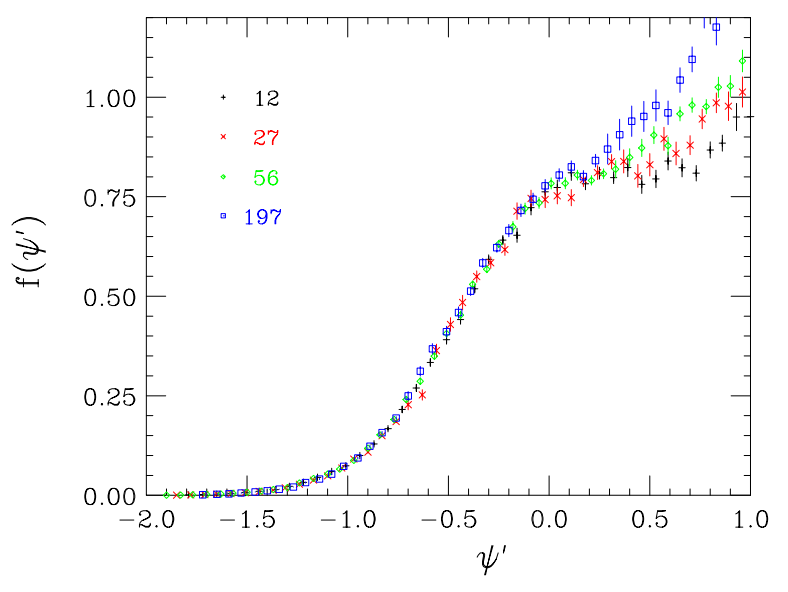

Figure 9: Illustration of scaling of second kind. The scaling functions for nuclei with mass number $12 \leq \mathrm{A} \leq 197$, obtained from the data of Ref. [55] at beam energy $E_{e}=3.6 \mathrm{GeV}$ and electron scattering angle $\theta_{e}=16 \mathrm{deg}$, corresponding to $|\mathbf{q}| \sim 1 \mathrm{GeV}$, are shown as a function of the variable $\psi^{\prime}=y / k_{F}[54]$.

\subsection{Final state interactions}

The occurrence of strong FSI in electron-nucleus scattering processes-not taken into account within the IA scheme-has long been experimentally established. The results of a number of $\left(e, e^{\prime} p\right)$ measurements, covering the kinematical domain corresponding to $0.5 \lesssim Q^{2} \lesssim 8.0(\mathrm{GeV} / \mathrm{c})^{2}$ [59-63], clearly show that the flux of outgoing protons is strongly suppressed with respect to the IA predictions. The observed attenuation, parametrized by the nuclear transparency $T_{A}$, ranges from 20$40 \%$ in Carbon to $50-70 \%$ in Gold, and becomes independent of momentum transfer for $Q^{2} \gtrsim 1 \mathrm{GeV}$. This behaviour is consistent with the results of theoretical studies of nuclear matter, providing clear cut evidence of the persistence of FSI at large momentum transfer [64].

Being only sensitive to interactions taking place within a distance $\sim 1 /|\mathbf{q}|$ of the primary vertex, the inclusive cross section at high momentum transfer is, in general, largely unaffected by FSI. However, the role of FSI turns out to be appreciable, or even dominant, in the low $\omega$ region, where the cross section obtained within the IA becomes very small.

Let us consider nuclear interactions at fixed beam energy and electron scattering angle in the purely nucleonic sector. As long as the sum over final states comprises a complete set, FSI do not affect the $\omega$-integrated cross section. Therefore, they can only give rise to two effects: (i) a shift in $\omega$, arising from the interaction of the struck nucleon with the mean field of the residual nucleus, and (ii) a redistribution of the strength-leading to a quenching of the quasi elastic peak and a corresponding enhancement of the tails-arising from NN scattering processes coupling $1 \mathrm{p} 1 \mathrm{~h}$ states to more complex final states.

Within the IPM, FSI can be described replacing the plane wave describing the struck nucleon in the final state with a wave function obtained from the solution of the Schrödinger equation involving a complex optical potential. This approach, referred to as Distorted Wave Impulse Approximation, or DWIA (see, e.g., Ref. [65]), can be generalised to allow for a consistent treatment of relativistic effects, and has been widely applied to both inclusive and exclusive processes [66-69]. In inclusive processes, however, there is no absorption, and FSI are usually described using a real optical potential. An alternative relativistic formalism employed for the description of inclusive processes is based on the expansion of the Green's function entering the definition of the target response tensor in eigenfunctions of a non-hermitian optical potential [70].

In the widely employed convolution approach [71, 72] the nuclear cross section is written in terms of the IA result according to

$$
\frac{d \sigma}{d \Omega_{e^{\prime}} d E_{e^{\prime}}}=\int d \omega^{\prime} \frac{d \sigma_{I A}}{d \Omega_{e^{\prime}} d E_{e^{\prime}}} F_{\mathbf{q}}\left(\omega-\omega^{\prime}\right)
$$

where the folding function, defined as

$$
F_{\mathbf{q}}(\omega)=\sqrt{T_{A}} \delta(\omega)+\left(1-\sqrt{T_{A}}\right) f_{\mathbf{q}}(\omega)
$$

embodies all FSI effects.

Equation (35) shows that the description of FSI involves (i) the nuclear transparency and (ii) the finite-width folding function $f_{\mathbf{q}}(\omega)$. Note that these quantities are both strongly affected by short-range correlations, since the repulsive core of the NN potential reduces the probability that the struck nucleon may interact with one of the spectator particles within a distance $\leq 1 \mathrm{fm}$ of the primary interaction vertex [73]. In the absence of FSI, $T_{A} \rightarrow 1$, implying that the residual nucleus is fully transparent to the struck nucleon, $f_{\mathbf{q}}(\omega) \rightarrow \delta(\omega)$, and the IA cross section of Eq. (21) is recovered.

As pointed out in Ref. [74], the convolution approach can be regarded as a generalisation of the spectral function formalism described in Secion 3.2, since the function $F_{\mathbf{q}}(\omega)$ turns out to be simply related to the spectral function describing the propagation of a nucleon in a continuum state. However, for large momentum transfer this quantity cannot be obtained using the non relativistic formalism.

Within the approach developed by the authors of Ref. [72], the folding function is derived within the eikonal approximation, which basically amounts to assuming that (i) the struck nucleon moves along a straight line with constant velocity, and (ii) the spectator nucleons are seen by the struck nucleon as a collection of fixed scattering centres. Under these assumptions, the elements entering the calculation of $F_{\mathbf{q}}(\omega)$ are the NN scattering amplitude, extracted from the measured cross sections, and the distribution of the spectator nucleons in coordinate space, that can be consistently obtained within NMBT using the same dynamical model employed for the description of the initial state.

The real part of the NN scattering amplitude is the source of the shift of the folded cross section of Eq. (34), with respect to the IA result. As shown in Ref. [24], this effect can be accurately parametrized in terms of a phenomenological real optical potential. On the other hand, the imaginary part-related to the total NN scattering cross section through the optical theorem-determines the shape of the function $f_{\mathbf{q}}(\omega)$.

It has to be pointed out that the $\mathrm{NN}$ cross section, driving the rescattering processes, is strongly influenced by the presence of the nuclear medium, which affects both the incoming flux and 


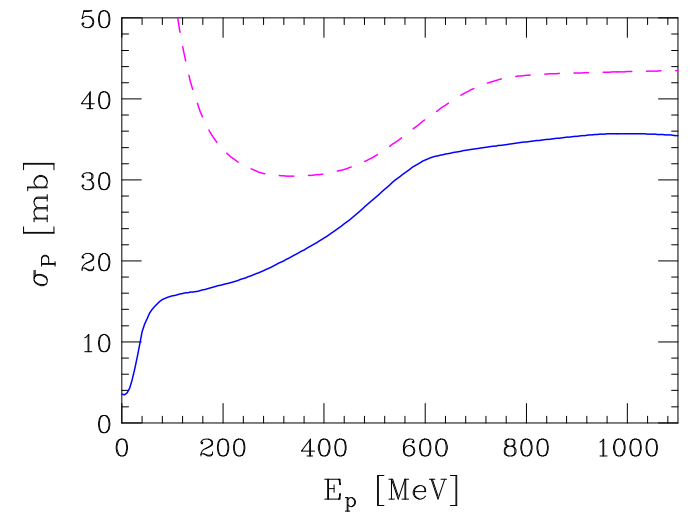

Figure 10: Total proton-neutron cross section as a function of the projectile kinetic energy in the Lab frame [74]. The dashed line shows the free space cross section, while the solid line has been obtained including medium modifications according to the procedure developed in Refs. [75, 76].

the available phase space. The medium modifications of the total cross section in the proton-neutron channel is illustrated by the results of Ref. [75], shown in Fig. 10.

\subsection{Two-nucleon currents and $2 p 2 h$ final states}

In addition to NN correlations in the initial and final states, interactions involving electromagnetic two-nucleon currents, arising from processes in which the photon couples to a meson exchanged between two interacting nucleons, also lead to the excitation of $2 \mathrm{p} 2 \mathrm{~h}$ final states. As an example, the simplest such processes contributing to the electron scattering cross section are depicted in Fig. 11.

The two-body currents are linked to the potential describing NN interactions through the continuity equation (3), establishing a relation between the nuclear Hamiltonian $H$ and the longitudinal component of the current $J^{\mu}$. As a consequence, the operator $J^{\mu}$ can be separated into model-dependent and modelindependent contributions, the latter being constrained by the NN potential [30].

As pointed out above, in the regime of low to moderate momentum transfer the nuclear matrix element of the two-nucleon current can be evaluated using realistic nuclear wave functions, obtained within the framework of NMBT, and a non relativistic reduction of the current operator, based on its expansion in powers of $|\mathbf{q}| / m$ [40]. The model-dependent component of the current, being transverse in nature, is not determined by the NN potential. Existing calculations typically take into account the isoscalar $\rho \pi \gamma$ and isovector $\omega \pi \gamma$ transition currents, as well as the isovector current associated with excitation of intermediate $\Delta$-isobar resonances. The two-body charge operators include the $\pi$-, $\rho$-, and $\omega$-meson exchange charge operators, the (isoscalar) $\rho \pi \gamma$ and (isovector) $\omega \pi \gamma$ couplings and the single-nucleon Darwin-Foldy and spin-orbit relativistic corrections [77].

The role of the two nucleon current in electron scattering is best illustrated by comparing the longitudinal and transverse contributions to the scaling function $F(y)$, defined by Eqs. (32) and (33).

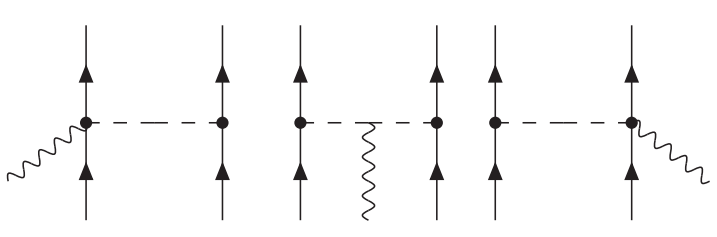

Figure 11: Diagrams depicting processes contributing to the electromagnetic two-nucleon current. Oriented lines correspond to nucleons, while the wavy and dashed lines are associated with the photons and the exchanged mesons, respectively.

It is important to recall that the occurrence of scaling of first kind provides a strong handle on the identification of the reaction mechanism, while the observation of scaling violations reveals the role played by processes beyond the IA. In this context, valuable information is provided by the scaling analysis of the longitudinal (L) and transverse (T) contributions to the measured cross sections [see Eq. (10)].

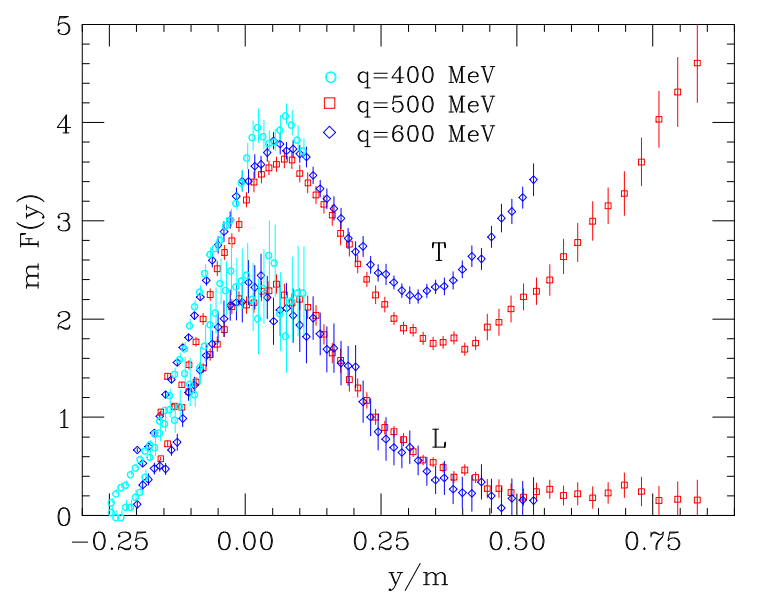

Figure 12: $y$-dependence of the longitudinal (L) and transverse (T) scaling functions of Carbon at $|\mathbf{q}|=400,500$, and $600 \mathrm{MeV}$ [78], obtained from the analysis of the data of Ref. [79]. Note that $y$ is given in units of the nucleon mass, $m$, and that the scaling function is multiplied by $m$, to obtain a dimensionless quantity.

Figure 12 shows the $y$-dependence of the $\mathrm{L}$ and $\mathrm{T}$ scaling functions obtained by the authors of Ref. [78] using the corresponding carbon responses, extracted from the cross sections measured at Saclay [79]. The onset of scaling is manifest in the region of the quasi free peak, corresponding to $y \sim 0$, where the data points at different momentum transfer tend to sit on top of one another as $|\mathbf{q}|$ increases. On the other hand, large scaling violations, mainly arising from non QE processes, such as resonance production, are clearly visible in the transverse channel at $y>0$, corresponding to $\omega>\omega_{\mathrm{QE}}$. In addition, the $\mathrm{T}$ scaling function turns out to be significantly larger than the $\mathrm{L}$ one, while within the IA picture-neglecting the small convection terms in the nucleon current- the $\mathrm{L}$ and $\mathrm{T}$ scaling functions are predicted to be identical (see, e.g., Ref. [80]).

The results of $a b$ initio Green's Function Monte Carlo (GFMC) calculations of the longitudinal and transverse responses of nuclei with $\mathrm{A} \leq 12$ [81, 82] provide convincing evidence that the pattern observed in Fig. 12 is driven by processes 
involving two-nucleon currents, whose contributions, while being negligible in the longitudinal channel, give rise to a significant enhancement of the transverse response.

The role of the two-body currents in determining the sum rules of the $\mathrm{L}$ and $\mathrm{T}$ responses, defined as

$$
S_{L}(|\mathbf{q}|)=\frac{1}{Z} \int_{\omega_{\text {th }}}^{\infty} d \omega R_{L}(|\mathbf{q}|, \omega)
$$

and

$$
S_{T}(|\mathbf{q}|)=\frac{2}{Z \mu_{p}+N \mu_{n}} \frac{m^{2}}{|\mathbf{q}|^{2}} \int_{\omega_{\mathrm{th}}}^{\infty} d \omega R_{T}(|\mathbf{q}|, \omega),
$$

has been thoroughly analysed by the authors of Ref. [81] using the GFMC approach. In the above equations, $R_{L}$ and $R_{T}$ are the response functions defined in Eq.(10), $\mu_{p}$ and $\mu_{n}$ are the proton and neutron magnetic moments, respectively, and the lower integration limit, $\omega_{\text {th }}$, corresponds to the threshold of inelastic scattering.

The numerical results of Ref. [81], including the $\mathrm{L}$ and $\mathrm{T}$ sum rules of ${ }^{3} \mathrm{He},{ }^{4} \mathrm{He}$ and ${ }^{6} \mathrm{Li}$ at momentum transfer $300 \leq$ $|\mathbf{q}| \leq 700 \mathrm{MeV}$, indicate that two-nucleon currents are responsible for $\mathrm{a} \sim 20-40 \%$ enhancement of of the $\mathrm{T}$ sum rule, while the typical contribution to $S_{L}$ is a $\sim 5 \%$ decrease. A similar pattern emerges from the analysis of Ref. [82], whose authors have computed the longitudinal and transverse responses of ${ }^{12} \mathrm{C}$.

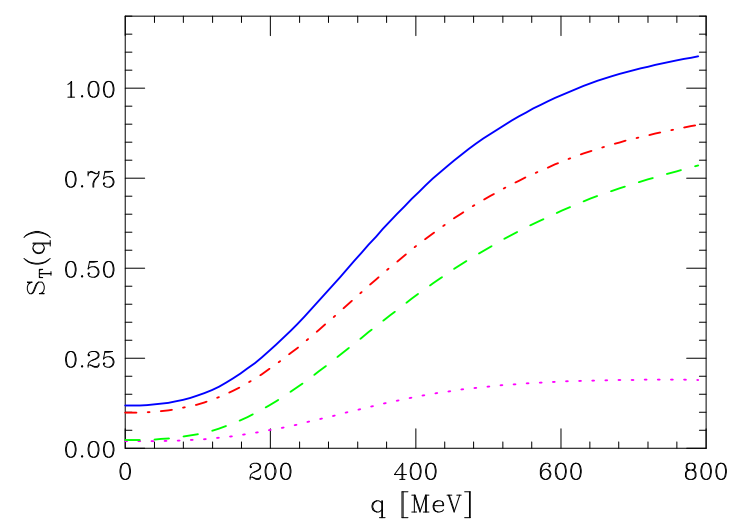

Figure 13: Sum rule of the electromagnetic response of carbon in the transverse channel, defined by Eq. (37). The dashed line shows the results obtained including the one-nucleon current only, while the solid line corresponds to the full calculation. The dot-dash line represents the sum rule computed neglecting interference terms, the contribution of which is displayed by the dotted line. The results are normalised so that the dashed line approaches unity as $|\mathbf{q}| \rightarrow \infty$ $[50]$.

As pointed out above, owing to the presence of NN correlations $2 \mathrm{p} 2 \mathrm{~h}$ final states can be excited in processes involving both one- and the two-body currents. Within the IA scheme, the contribution of the one-body current can be taken into account using spectral functions derived from realistic nuclear models, in which the ground state has non vanishing overlaps with the two hole-one particle states of the residual system [41]. On the other hand, the discussion of Section 3.2 implies that all models based on the mean field approximation fail to meet this requirement.
A consistent treatment of the one- and two-nucleon current contributions to the nuclear cross section in the $2 \mathrm{p} 2 \mathrm{~h}$ sector requires that interference between the corresponding amplitudes - including the one associated with the excitation of $2 \mathrm{p} 2 \mathrm{~h}$ final states in the aftermath of a rescattering of the knocked out particle, to be discussed below-be carefully taken into account.

The role of interference terms in determining the transverse electromagnetic response of ${ }^{12} \mathrm{C}$ has been recently analysed within the GFMC approach. The results of this study, displayed in Fig. 13, clearly show that interference is the source of a sizeable fraction of the sum rule. At momentum transfer $|\mathbf{q}| \gtrsim 300$ $\mathrm{MeV}$, its contribution turns out to be comparable to-in fact even larger than - the one arising from the squared matrix element of the two-nucleon current [50].

A fully consistent description of one- and two-body current contributions to the nuclear cross sections in the region in which the non relativistic approximation is expected to break down involves serious difficulties. Existing calculations have been mainly carried out using diagrammatic approaches, based on simplified descriptions of the the nuclear initial and final states, obtained from either the RFGM or more advanced implementations of the mean field approximation [83, 84].

A novel approach, recently proposed in Refs. [50, 85], is based on a generalisation of the factorisation ansatz described in Section 3.1. The $2 \mathrm{p} 2 \mathrm{~h}$ final state is written in the form [compare to Eq. (13)]

$$
|X\rangle=\left|\mathbf{p p}^{\prime}\right\rangle \otimes\left|\mathrm{R}_{A-2}, \mathbf{p}_{\mathrm{R}}\right\rangle,
$$

where the states $\left|\mathbf{p p}^{\prime}\right\rangle$ and $\left|\mathbf{R}_{A-2}, \mathbf{p}_{\mathrm{R}}\right\rangle$ describe two non interacting nucleons, carrying momenta $\mathbf{p}$ and $\mathbf{p}^{\prime}$, and the recoiling (A - 2)-particle spectator system, respectively.

Using Eq. (38) and following the procedure described in Section 3.1, the nuclear matrix element of the two-nucleon current can be written in terms of two-body matrix elements according to

$$
\left\langle 0\left|J_{2}^{\mu}\right| X\right\rangle=\frac{m}{\sqrt{E_{p} E_{p^{\prime}}}} \int d^{3} k d^{3} k^{\prime} \mathcal{M}_{\mathbf{R}}\left(\mathbf{k}, \mathbf{k}^{\prime}\right) \sum_{j>i}\left\langle\mathbf{p p}^{\prime}\left|j_{i j}^{\mu}\right| \mathbf{k} \mathbf{k}^{\prime}\right\rangle,
$$

with the amplitude $\mathcal{M}_{\mathrm{R}}\left(\mathbf{k}, \mathbf{k}^{\prime}\right)$ given by

$$
\mathcal{M}_{\mathrm{R}}\left(\mathbf{k}, \mathbf{k}^{\prime}\right)=\left\{\left\langle\mathrm{R}_{(A-2)}, \mathbf{p}_{\mathrm{R}}\left|\otimes\left\langle\mathbf{k} \mathbf{k}^{\prime}\right|\right\} \mid 0\right\rangle .\right.
$$

Within the scheme outlined in Eqs. (38)-(40), the nuclear amplitude $\mathcal{M}_{\mathrm{R}}\left(\mathbf{k}, \mathbf{k}^{\prime}\right)$ turns out to be independent of $\mathbf{q}$, and can therefore be obtained within NMBT. On the other hand, the two-nucleon matrix element between free nucleon states can be evaluated using the fully relativistic expression of the current.

The connection with the spectral function formalism becomes apparent noting that the two-nucleon spectral function $P\left(\mathbf{k}, \mathbf{k}^{\prime}, E\right)$, yielding the probability of removing two nucleons with momenta $\mathbf{k}$ and $\mathbf{k}^{\prime}$ from the nuclear ground state, leaving the residual system with excitation energy $E$, is defined as [86]

$$
P\left(\mathbf{k}, \mathbf{k}^{\prime}, E\right)=\sum_{\mathrm{R}}\left|\mathcal{M}_{\mathrm{R}}\left(\mathbf{k}, \mathbf{k}^{\prime}\right)\right|^{2} \delta\left(E+E_{0}-E_{\mathrm{R}}\right),
$$


with $\mathcal{M}_{\mathrm{R}}\left(\mathbf{k}, \mathbf{k}^{\prime}\right)$ given by Eq. (40).

The two-nucleon spectral function of uniform and isospin symmetric nuclear matter at equilibrium density has been calculated by the authors of Ref. [86] using CBF perturbation theory and a realistic Hamiltonian. The resulting relative momentum distribution, defined as

$$
n_{\text {rel }}(\mathbf{Q})=4 \pi|\mathbf{Q}|^{2} \int d^{3} K n\left(\frac{\mathbf{K}}{2}+\mathbf{Q}, \frac{\mathbf{K}}{2}-\mathbf{Q}\right)
$$

where $\mathbf{K}=\mathbf{k}+\mathbf{k}^{\prime}, \mathbf{Q}=\left(\mathbf{k}-\mathbf{k}^{\prime}\right) / 2$, and

$$
n\left(\mathbf{k}, \mathbf{k}^{\prime}\right)=\int d E P\left(\mathbf{k}, \mathbf{k}^{\prime}, E\right)
$$

is shown by the solid line of Fig. 14. Comparison with the prediction of the Fermi Gas model, represented by the dashed line, clearly illustrates the significance of correlation effects.

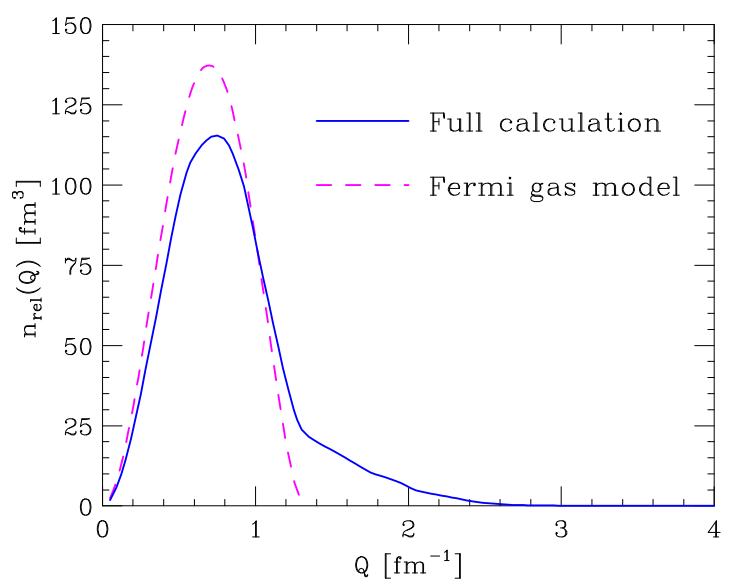

Figure 14: Relative momentum distribution of a nucleon pair in isospin symmetric nuclear matter at equilibrium density [86].

\subsection{Collective excitations}

At low momentum transfer, the interaction with the beam particle may involve more than one nucleon, and give rise to long-range correlations leading to the appearance of collective excitations of the target nucleus.

The contribution of collective excitations can be approximated writing the final state appearing in Eq. (7) as a superposition of $1 \mathrm{p} 1 \mathrm{~h}$ states. The resulting expression of the nuclear response involves the propagator of the particle-hole pair excited at the the interaction vertex, $\Pi(\mathbf{q}, \omega)$, carrying momentum and energy $\mathbf{q}$ and $\omega$.

Within the commonly used Random Phase Approximation (RPA), $\Pi(\mathbf{q}, \omega)$ is obtained from an integral equation, which allows to take into account the contributions of the so called ring diagrams to all orders. Most existing calculations have been performed within a theoretical framework based on the IPM, in which the particle-hole interaction is described in terms of perturbative meson exchange, augmented by a set of phenomenological parameters. This scheme has been also extended to include contributions involving $\Delta$-resonance production [87, 88].
In recent years, a significant effort has been devoted to the development of a treatment of RPA correlations based on more realistic dynamical models [89, 90]. In Refs. [91, 92], the weak responses of nuclear matter at low momentum transfer have been computed using an effective particle-hole interaction obtained from a phenomenological Hamiltonian including twoand three-nucleon potentials. The results of these studies indicate that - as it was to be expected on the basis of very general quantum mechanical considerations - the effects of collective excitations, while being large at $|\mathbf{q}|<100 \mathrm{MeV}$, become less and less important with decreasing momentum transfer, and turn out to be vanishingly small at $|\mathbf{q}| \gtrsim 400 \mathrm{MeV}$ [90].

\subsection{Comparison to data}

The ability to account for electron scattering data is the obvious prerequisite to be fulfilled by any model of neutrino interactions. Below, we provide some representative comparisons between the results of the approaches outlined in the previous Sections and the available empirical information, obtained from the measured cross sections.

Non relativistic ab initio calculations of the electromagnetic responses of few-nucleon systems with $A \leq 4$, performed using realistic nuclear Hamiltonians, have reached a remarkable degree of accuracy, and provide a good description of a large body of data (see, e.g., Refs. [35-39]). In this review, however, we will focus on the heavier nuclei employed to detect neutrino interactions, such as carbon, oxygen and argon.

The electromagnetic longitudinal and transverse responses of carbon, defined as in Eq (10), have been analysed using the GFMC formalism. The available results include the Euclidean responses, related to the corresponding $\omega$-space responses by Laplace transformation [93], as well as the sum rules defined by Eqs.(36) and (37) [82, 94].

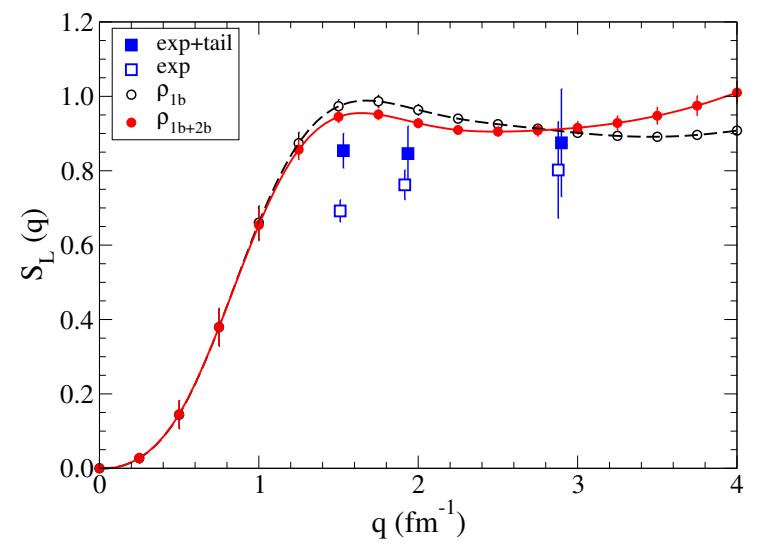

Figure 15: Longitudinal sum rule of ${ }^{12} \mathrm{C}$, computed by the authors of Ref. [94] using the GFMC formalism. The solid line has been obtained using the full current operator of Eq.(4), while the dashed line does not take into account the contribution of two-nucleon terms. The experimental data, with (full squares) and without (open squares) tail correction, correspond to the response functions resulting from the analysis of Ref. [95].

Figures 15 and 16 show the longitudinal and transverse sum rules of carbon computed by the authors of Ref. [94]. Theoretical results turn out to be in satisfactory agreement with the data, 


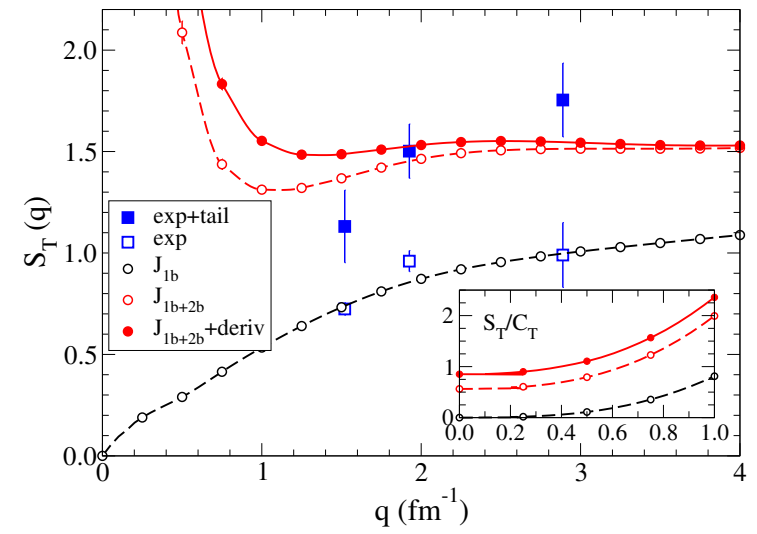

Figure 16: Same as in Fig. 15, but for the transverse sum rule. The inset shows the low- $|\mathbf{q}|$ behaviour obtained removing from the definition of $S_{T}$, Eq. (37), the $\sim 1 /|\mathbf{q}|^{2}$ divergent coefficient [94].

corrected to take into account the contribution of the region of large $\omega$ not covered by the experiments (see Ref. [94] and references therein). In addition, a comparison between the solid and dashed lines confirms that the two-nucleon currents, while playing a nearly negligible role in the longitudinal channel, provide a large contribution to the transverse sum rule. A recent, more accurate, analysis has shown that the agreement between the GFMC longitudinal sum rule of carbon and the data can be further improved taking into account the contributions of the low-lying $J^{\pi}=2^{+}, 0_{2}^{+}$(Hoyle), and $4^{+}$states [82].

The main limitation of the sum rules is the lack of information on the energy distribution of strength. As a consequence, the analysis based on sum rules does not answer the question of whether the excess of transverse strength arising fom interactions involving the two-nucleon currents occurs mostly at large $\omega$-well beyond the quasi-elastic peak-or it is also found at $\omega \approx \omega_{\mathrm{QE}}$.

The inversion of the Euclidean response, needed to retrieve the energy dependence, is long known to involve severe difficulties. A groundbreaking result has been recently reported in Refs. [82, 93], whose authors exploited the maximum entropy technique to obtain the electromagnetic longitudinal and transverse responses of ${ }^{4} \mathrm{He}$ and ${ }^{12} \mathrm{C}$.

Figures 17 and 18 show the $\omega$ dependence of the transverse and longitudinal responses of ${ }^{12} \mathrm{C}$ at $|\mathbf{q}|=570 \mathrm{MeV}$, obtained by inverting the corresponding euclidean response [82]. It appears that the GFMC approach provides a quantitative account of the data in the region in which the description in terms of purely nucleonic degrees of freedom is applicable. The contribution arising from processes involving two-nucleon currents is sizeable in the transverse channel, and extends over the whole $\omega$ range. On the other hand, it turns out to be nearly negligible in the longitudinal channel.

The results of the approach based on the IA and the spectral function formalism, corrected to take into account the effects of FSI within the convolution scheme described in Section 3.4 [24], are displayed by the solid lines of Fig. 19. It clearly ap-

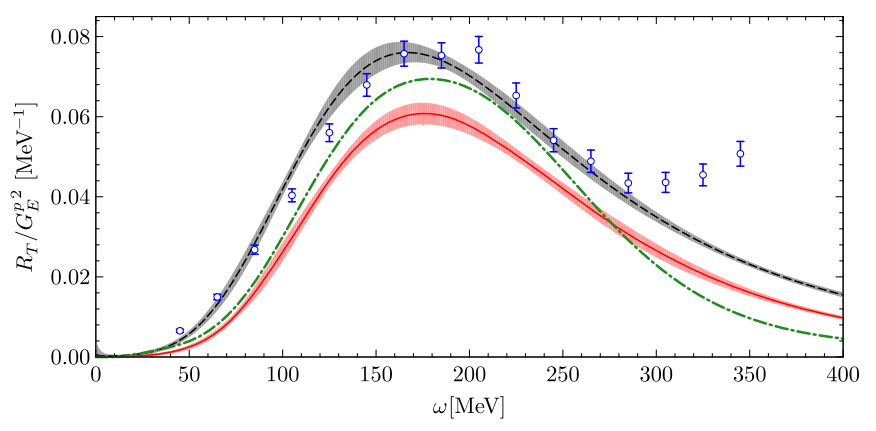

Figure 17: Transverse electromagnetic response functions of ${ }^{12} \mathrm{C}$ at $|\mathbf{q}|=570$ $\mathrm{MeV}$, computed within the GFMC approach [82]. The dashed and solid lines have been obtained using the full nuclear current operator and neglecting the two-body terms, respectively, while the dot-dash line shows the results of a PWIA calculation. The shaded areas represent the uncertainty associated with the inversion of the euclidean response. The data is taken from Ref. [95]

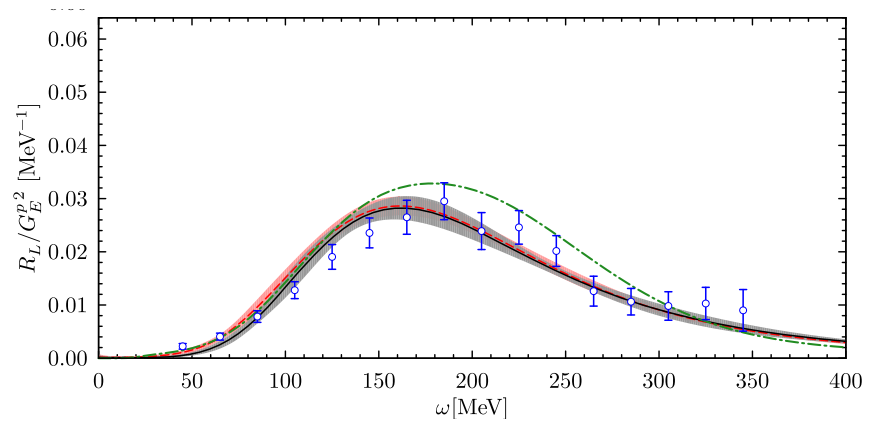

Figure 18: Longitudinal electromagnetic response functions of ${ }^{12} \mathrm{C}$ at $|\mathbf{q}|=570$ $\mathrm{MeV}$, computed within the GFMC approach [82]. The solid and dashed lines have been obtained using the full nuclear current operator and neglecting the two-body terms, respectively, while the dot-dash line shows the results of a PWIA calculation. The shaded areas represent the uncertainty associated with the inversion of the euclidean response. The data is taken from Ref. [95]

pears that the description of nuclear dynamics based on a realistic Hamiltonian and nuclear many-body theory, which does not include any adjustable parameters, provides a quantitative description of the data in the region in which QE scattering is dominant. On the other hand, the dotted lines show that the RFGM, while yielding an acceptable account of few measured cross sections, conspicuously fails to explain the data over the entire ranges of beam energy and scattering angle.

Although the results of Ref. [24] are limited to the QE sector, the spectral function formalism provides a framework for the consistent description of both elastic and inelastic singlenucleon knock out processes. Moreover, as pointed out above, it can be generalised to include both FSI and the contributions of processes involving two-nucleon currents. Figure 20 shows a comparison between the measured electron-carbon cross sections of Refs. [32, 79] and the results of the calculations discussed in Ref. [98], in which MEC and FSI are both taken into account.

Besides the $a b$ initio approaches based on the Hamiltonian of Eq. (2), a number of schemes based on different-and somewhat simplified - descriptions of nuclear structure and dynamics have been employed to study electron-nucleus scattering.

As an example, Fig. 21 shows the superscaling functions at 

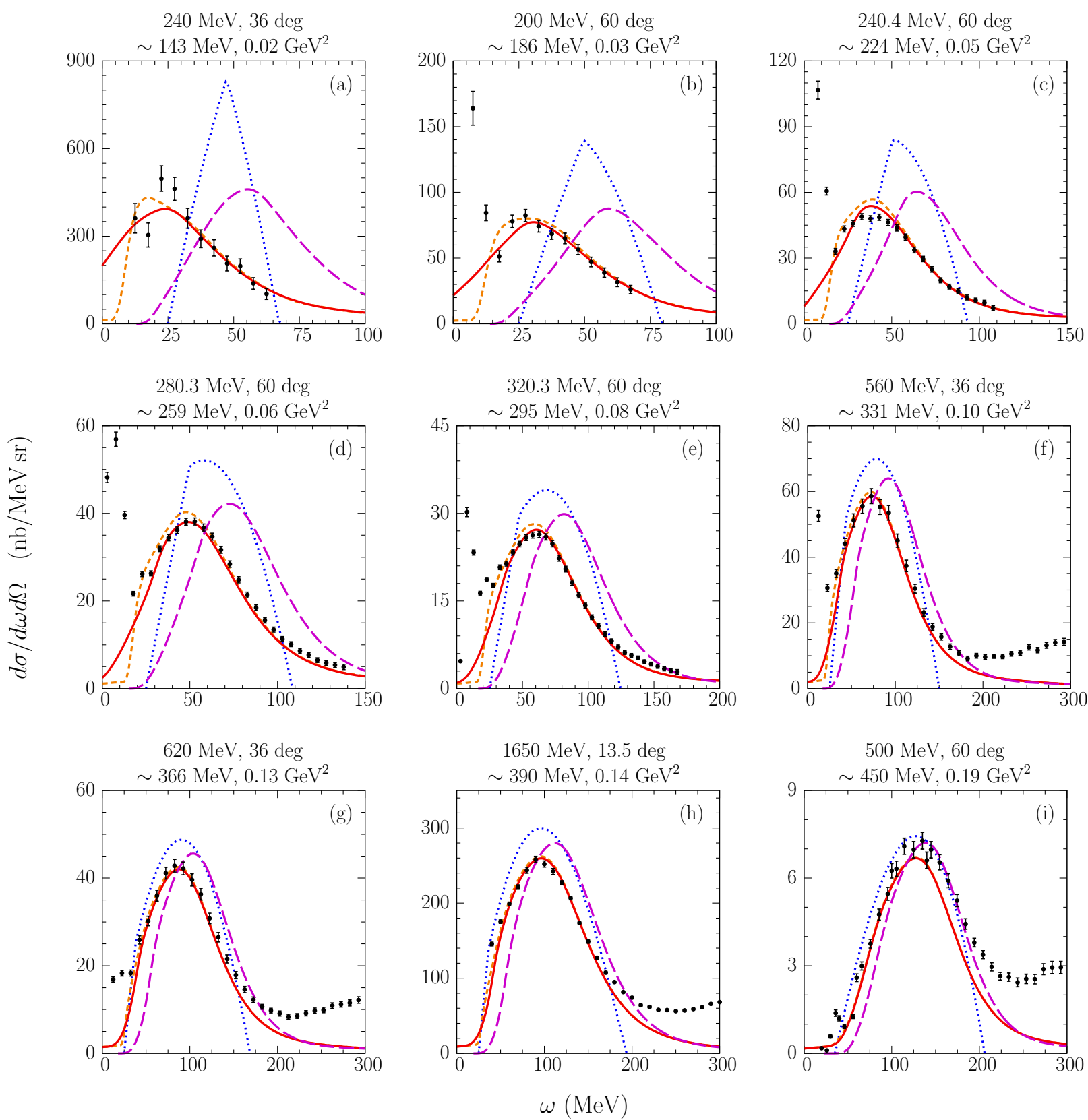

Figure 19: Double differential electron-carbon cross sections in the QE channel, computed by the authors of Ref. [24] within the spectral function approach, compared to the data of Ref. [79, 96,97]. The solid lines correspond to the result of the full calculation, whereas the long-dashed lines have been obtained neglecting FSI. The difference between the solid and short-dashed lines illustrates the effect of using alternative treatments of Pauli blocking. For comparison, the prediction of the RFGM are also shown, by the dotted lines. The panels are labeled according to beam energy, scattering angle, and values of $|\mathbf{q}|$ and $Q^{2}$ at the quasielastic peak.

$|\mathbf{q}|=500$ and $1000 \mathrm{MeV}$, obtained using the mean field approximation for the initial state and two alternative approaches for the treatment of FSI. The curves labeled GF1 and GF2 have been obtained from the Green's function formalism (see Section 3.4) and two different complex optical potentials, while the curve labeled RMF corresponds to calculations carried out within the relativistic mean field scheme, using the same real (scalar and vector) potentials to describe both bound and scattering nucleon states. On the other hand, the curve labelled rROP shows the results obtained using the real part of the rel- ativistic optical potential to determine the outgoing nucleon wave function. For comparison, the result obtained neglecting FSI altogether are also shown, by the line labelled RPWIA [99].

The phenomenological superscaling analysis has been recently extended with the inclusion of additional information, obtained from studies carried out within the RMF approach, which allow to pin down the nuclear responses in the isoscalar and isovector channels [100]. The model described in Ref. [100], referred to as SuSav2, has been further developed to take into account inelastic channels, and augmented 


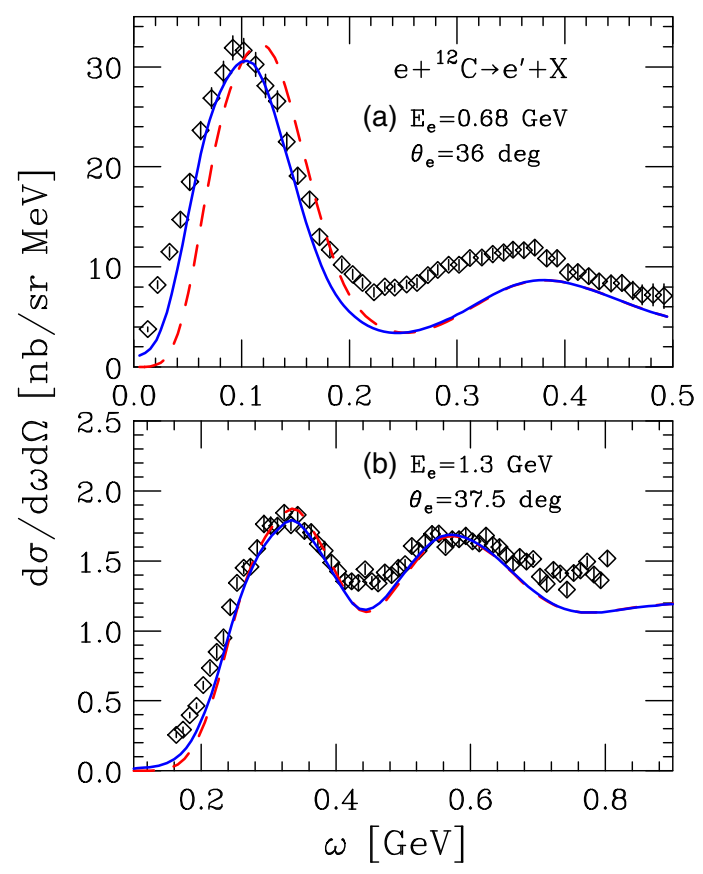

Figure 20: (a): double differential electron-carbon cross section at beam energy $E_{e}=680 \mathrm{MeV}$ and scattering angle $\theta_{e}=36 \mathrm{deg}$ [98]. The solid and dashed lines have been obtained from the spectral function approach, with and without inclusion of FSI. The experimental data are taken from Ref. [79]. (b): same as (a) but for $E_{e}=1300 \mathrm{MeV}$ and $\theta_{e}=37.5 \mathrm{deg}$. The experimental data are taken from Ref. [32].

considering the contributions of processes involving MEC, described according to the RFGM [101]. The results displayed in Fig. 22 show that the hybrid procedure derived in Ref. [101], dubbed SuSav2-MEC, describes the measured electron-carbon cross sections with accuracy comparable to that obtained using the spectral function formalism.

The authors of Refs. [87, 88] developed diagrammatic approaches to study the inclusive electron nucleus cross section, in which the effects of long range RPA correlations is explicitly taken into account, and found to be significant. Within these models, in which the description of the target ground state is based on the RFGM, interaction effects are included at the level of perturbative meson exchange, and short-range correlations are taken into account through phenomenological modifications of the $\mathrm{NN}$ amplitudes. As an example, Fig. 3.7 shows the electron-carbon cross sections reported by the authors of Ref. [88] for two kinematical setups, corresponding to $|\mathbf{q}| \sim 600 \mathrm{MeV}$ (upper panel) and $\sim 400 \mathrm{MeV}$ (lower panel). The model of Ref. [88] has been also applied to the description of a variety of semi-inclusive electron scattering processes, including $\left(e, e^{\prime} N\right),\left(e, e^{\prime} N N\right)$, and $\left(e, e^{\prime} \pi\right)$ [102].

\section{The flux-integrated neutrino-nucleus cross section}

For definiteness, we will consider charged-current neutrinonucleus interactions at fixed neutrino energy. The formalism discussed in this Section can be readily generalized to the case of neutral current interactions (see, e.g., Ref. [103]).
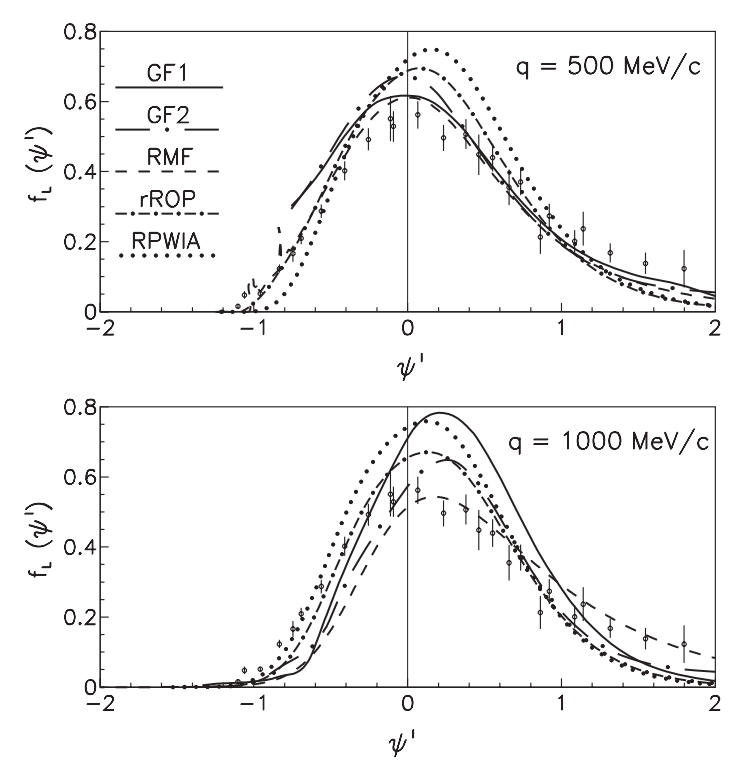

Figure 21: Superscaling functions computed by the authors of Ref. [99]. The meaning of the labels is explained throughout the text.
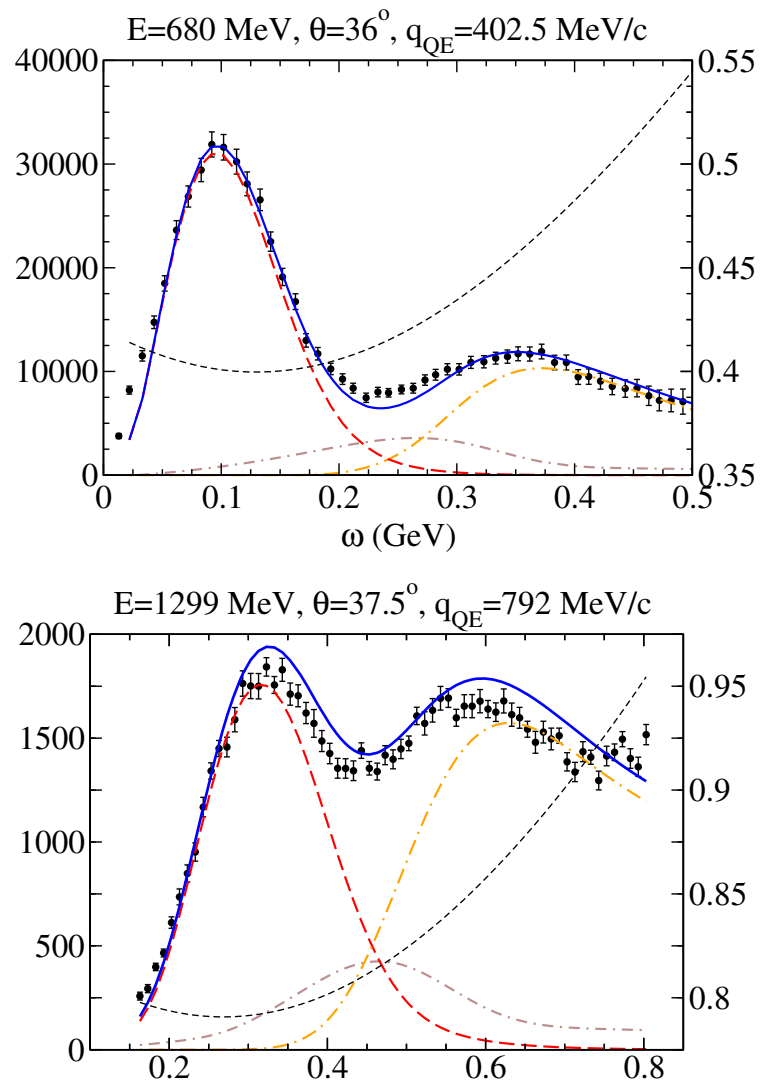

Figure 22: Comparison between the measured electron-carbon cross sections of Refs. [79] (upper panel) and [32] (lower panel) and the results of the SuSav2MEC approach of Ref. [101], represented by the solid lines. The vertical axis on the left corresponds to the double-differential cross section in units of $\mathrm{nb} / \mathrm{sr} / \mathrm{GeV}$. 

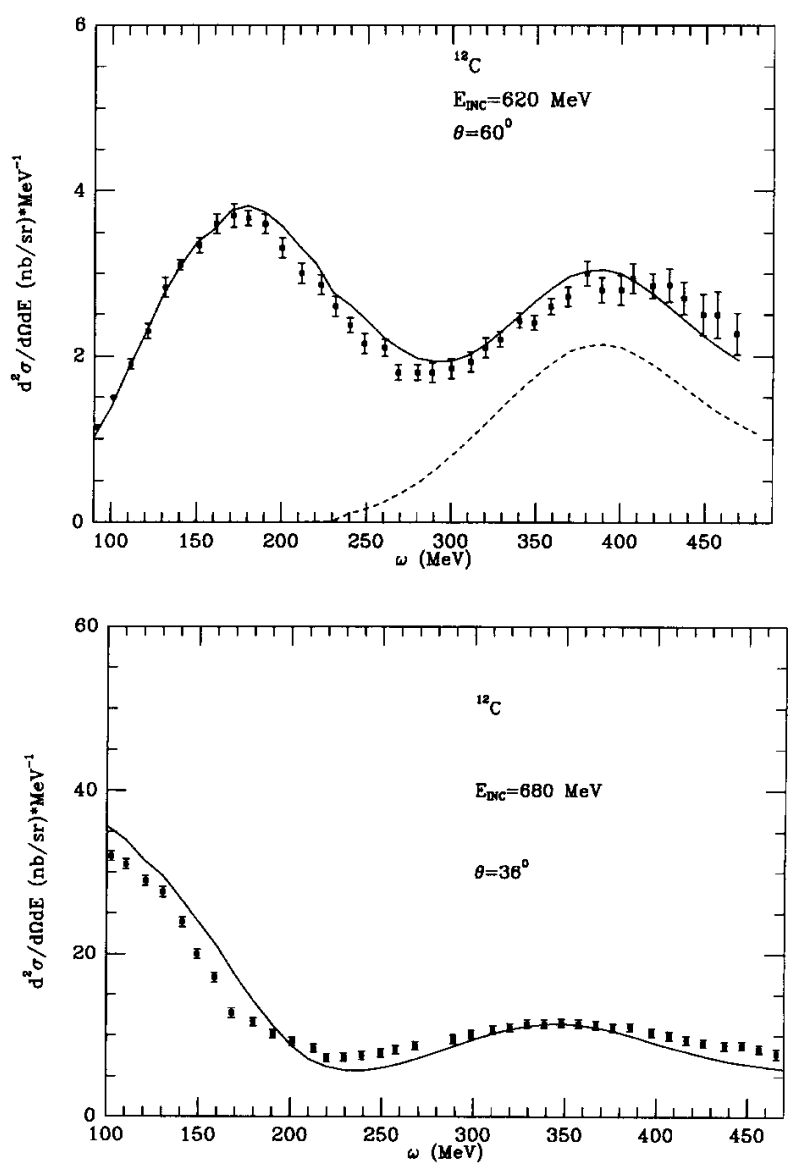

Figure 23: Electron-carbon cross sections at $E_{e}=620 \mathrm{MeV}$ and $\theta_{e}=60 \mathrm{deg}$ (upper panel) and $E_{e}=680 \mathrm{MeV}$ and $\theta_{e}=36 \mathrm{deg}$ (lower panel) obtained from the model of Ref. [88]. The data points are taken from Ref. [79].

The double differential cross section of the process [compare to Eq. (1)]

$$
v_{\ell}+A \rightarrow \ell^{-}+X
$$

can be written in the form (see, e.g., Ref. [104])

$$
\frac{d^{2} \sigma}{d \Omega_{\ell} d E_{\ell}}=\frac{G_{F}^{2} V_{u d}^{2}}{16 \pi^{2}} \frac{\left|\mathbf{k}_{\ell}\right|}{\left|\mathbf{k}_{v}\right|} L_{\lambda \mu} W^{\lambda \mu} .
$$

Here $k_{v} \equiv\left(E_{v}, \mathbf{k}_{v}\right)$ and $k_{\ell} \equiv\left(E_{\ell}, \mathbf{k}_{\ell}\right)$ are the four momenta carried by the incoming neutrino and the outgoing charged lepton, respectively, $G_{F}$ is the Fermi constant and $V_{u d}$ is the CKM matrix element coupling $u$ and $d$ quarks. Neglecting the term proportional to $m_{\ell}^{2}$, where $m_{\ell}$ is the mass of the charged lepton, the tensor $L^{\lambda \mu}$ can be cast in the form

$$
L^{\lambda \mu}=8\left[k_{\ell}^{\lambda} k_{v}^{\mu}+k_{v}^{\lambda} k_{\ell}^{\mu}-g^{\lambda \mu}\left(k_{\ell} \cdot k_{v}\right)-i \varepsilon^{\lambda \mu \alpha \beta} k_{\ell \beta} k_{v \alpha}\right],
$$

where $\varepsilon^{\lambda \mu \alpha \beta}$ is the fully antisymmetric Levi-Civita tensor.

The target tensor is written as in Eq. (7), replacing the electromagnetic current with the weak charged current. Within the IA scheme, it reduces to

$$
W^{\lambda \mu}=N \int d^{3} k d E \frac{M}{E_{k}} P(\mathbf{k}, E) W_{n}^{\lambda \mu},
$$

where the tensor $\mathcal{W}_{n}^{\lambda \mu}$ describes the interaction of a free neutron of four momentum $k$ at four momentum transfer $\tilde{q} \equiv(\tilde{\omega}, \mathbf{q})$, with $\tilde{\omega}$ given by Eq. (20). Its most general expression can be written in terms of five structure functions according to

$$
\begin{aligned}
W^{\lambda \mu}=-g^{\lambda \mu} W_{1}+\frac{k^{\lambda} k^{\mu}}{m^{2}} W_{2} & -i \epsilon^{\lambda \mu \varrho \sigma} \frac{k_{\varrho} k_{\sigma}}{m^{2}} W_{3} \\
& +\frac{q^{\lambda} q^{\mu}}{m^{2}} W_{4}+\frac{k^{\lambda} q^{\mu}+k^{\lambda} q^{\mu}}{m^{2}} W_{5} .
\end{aligned}
$$

Note that in scattering processes involving isolated nucleons, after contraction of $\mathcal{W}^{\lambda \mu}$ with the lepton tensor $L_{\lambda \mu}$, the structure functions $W_{4}$ and $W_{5}$ give vanishing contributions to the cross section. Owing to the replacement $q \rightarrow \tilde{q}$ in the arguments of $W^{\lambda \mu}$, in neutrino-nucleus scattering this is no longer the case. However, the results of numerical calculations suggest that the contributions of the terms involving $W_{4}$ and $W_{5}$ are small, and can be safely neglected [104].

From Eqs. (46) and (48) one obtains

$$
L^{\lambda \mu} W_{\lambda \mu}=16 \sum_{i} W_{i}\left(\frac{A_{i}}{m^{2}}\right),
$$

the kinematical factors $A_{i}$ being given by

$$
\begin{aligned}
& A_{1}=m^{2}\left(k \cdot k^{\prime}\right), \\
& A_{2}=(k \cdot p)\left(k^{\prime} \cdot p\right)-\frac{A_{1}}{2}, \\
& A_{3}=(k \cdot p)\left(k^{\prime} \cdot \tilde{q}\right)-(k \cdot \tilde{q})\left(k^{\prime} \cdot p\right), \\
& A_{4}=(k \cdot \tilde{q})\left(k^{\prime} \cdot \tilde{q}\right)-\frac{\tilde{q}^{2}}{2} \frac{A_{1}}{m^{2}}, \\
& A_{5}=(k \cdot p)\left(k^{\prime} \cdot \tilde{q}\right)+\left(k^{\prime} \cdot p\right)(k \cdot \tilde{q})-(\tilde{q} \cdot p) \frac{A_{1}}{m^{2}} .
\end{aligned}
$$

The flux-integrated double differential neutrino-nucleus cross section, defined as

$$
\frac{d \sigma}{d T_{\ell} d \cos \theta_{\ell}}=\frac{1}{N_{\Phi}} \int d E_{\nu} \Phi\left(E_{v}\right) \frac{d \sigma}{d E_{\nu} d T_{\ell} d \cos \theta_{\ell}},
$$

where $T_{\ell}=E_{\ell}-m_{\ell}$ is the kinetic energy of the outgoing charged lepton and

$$
N_{\Phi}=\int d E_{v} \Phi\left(E_{v}\right)
$$

can be readily obtained from the above equations, yielding the expression of the double differential cross section at fixed neutrino energy derived within the IA.

Equations (45)-(47) show that, within the scheme based on the IA, the calculation of the neutrino-nucleus cross section requires two ingredients: (i) the structure functions describing the elementary neutrino-nucleon interactions and (ii) the spectral function, describing the properties of the nuclear initial state. It follows that, to the extent to which the structure functions $W_{i}$ are known, the spectral function formalism provides a unified framework, suitable to describe neutrino-nucleus scattering in different kinematical regimes, in which different reaction mechanisms dominate. 
In Section 4.1 we report the explicit expression of the structure functions relevant to $\mathrm{CC} \mathrm{QE}$ interactions, which are the ones exploited for oscillation analyses. For completeness, the corresponding structure functions for the resonance production and deep inelastic scattering channels are briefly discussed in Sections 4.2 and 4.3 .

Owing to the moderate value of the mean energy of the neutrino flux of Fig. 3, $\left\langle E_{v}\right\rangle \sim 800 \mathrm{MeV}$, the MiniBooNE CC QE sample accounts for $\sim 60 \%$ of the total cross section, the remaining $\sim 40 \%$ being associated with inelastic processes. On the other hand, the DUNE neutrino flux has its maximum at $E_{v} \sim 2.5 \mathrm{GeV}$ and exhibits a long high-energy tail [105]. As a consequence, the fractions of $\mathrm{CC} \mathrm{QE}$, resonance production and deep inelastic events turn out to be $\sim 5 \%, \sim 35 \%$ and $\sim 60 \%$, respectively, and the identification of the relevant signal will require a fully quantitative understanding of the background of non CC QE interactions [106].

It has to be pointed out that, while in this review we mainly focus on the scheme based on the IA and the spectral function formalism ${ }^{4}$, a unified description of nuclear effects has been also developed within a completely different approach, based on transport theory. This approach, thoroughly described in Ref. [107], provides the conceptual framework underlying the Giessen Boltzmann-Uehling-Uhlenbeck (GiBUU) event generator, extensively applied to a variety of processes, ranging from pion-induced nuclear reactions to heavy-ion collisions and photon- and lepton-nucleus scattering.

\subsection{Quasielastic scattering}

In the $\mathrm{CC} \mathrm{QE}$ channel, the structure functions involve the energy conserving $\delta$-function enforcing the condition that the scattering process be elastic. Therefore, they can be conveniently written in the form

$$
W_{i}=\widetilde{W}_{i} \delta\left(\tilde{\omega}+\frac{\tilde{q}^{2}}{2 M}\right)
$$

with the $\widetilde{W}_{i}$ determined by the matrix elements of the weak nucleon current. Exploiting the CVC and PCAC hypoteses, the resulting structure functions, can be written in terms of the vector form factors, $F_{1}$ and $F_{2}$, and the axial-vector form factor, $F_{A}$, according to

$$
\begin{aligned}
& \widetilde{W}_{1}=2\left[F_{A}^{2}(1+\tau)+\tau\left(F_{1}+F_{2}\right)^{2}\right], \\
& \widetilde{W}_{2}=2\left[F_{A}^{2}+F_{1}^{2}+\tau F_{2}^{2}\right], \\
& \widetilde{W}_{3}=2 F_{A}\left(F_{1}+F_{2}\right), \\
& \widetilde{W}_{4}=\left[F_{2}^{2}(1+\tau)-2 F_{2}\left(F_{1}+F_{2}\right)\right] / 2, \\
& \widetilde{W}_{5}=W_{2} / 2,
\end{aligned}
$$

with $\tau=-\tilde{q}^{2} / 4 m^{2}$.

The form factors appearing in the vector current, $F_{1}\left(q^{2}\right)$ and $F_{2}\left(q^{2}\right)$, are obtained from the measured proton and neutron

\footnotetext{
${ }^{4}$ Note that this scheme provides the basis of a variety of different models, including the RFGM.
}

electric and magnetic form factors, $G_{E}^{N}$ and $G_{M}^{N}(N=p, n)$, through the relations

$$
F_{i}\left(q^{2}\right)=F_{i}^{p}\left(q^{2}\right)-F_{i}^{n}\left(q^{2}\right)
$$

with $i=1,2$ and

$$
\begin{aligned}
& F_{1}^{N}\left(q^{2}\right)=\frac{1}{(1-\tau)}\left[G_{E}^{N}\left(q^{2}\right)-\tau G_{M}^{N}\left(q^{2}\right)\right], \\
& F_{2}^{N}\left(q^{2}\right)=\frac{1}{(1-\tau)}\left[-G_{E}^{N}\left(q^{2}\right)+G_{M}^{N}\left(q^{2}\right)\right] .
\end{aligned}
$$

While more refined parametrisations of the large body of electron scattering data are available [108, 109], the form factors $G_{E}^{N}$ and $G_{M}^{N}$ are often written in the simple dipole approximation

$$
\begin{aligned}
& G_{E}^{p}\left(q^{2}\right)=\left(1-\frac{q^{2}}{m_{V}^{2}}\right)^{-2}, \quad G_{E}^{n}\left(q^{2}\right)=0, \\
& G_{M}^{N}\left(q^{2}\right)=\mu_{N}\left(1-\frac{q^{2}}{m_{V}^{2}}\right)^{-2},
\end{aligned}
$$

with $m_{V}^{2}=0.71 \mathrm{GeV}^{2}$. The axial form factor, $F_{A}$, is also written in the same form

$$
F_{A}\left(q^{2}\right)=g_{A}\left(1-\frac{q^{2}}{m_{A}^{2}}\right)^{-2}
$$

The axial coupling constant, $g_{A}=-1.2761_{-17}^{+14}$, is obtained from neutron $\beta$-decay [110], while the axial mass determined from elastic neutrino- and antineutrino-nucleon scattering, charged pion electro-production off nucleons and muon capture on the proton is $m_{A}=1.03 \mathrm{GeV}[111,112]$.

Note that in Eq. (54) the contributions involving the pseudoscalar form factor, $F_{P}$, have been neglected. This approximation is largely justified, except for the case of $v_{\tau}$ scattering.

\subsection{Resonance production}

The generalisation of the formalism summarised in the previous section to describe resonance production, driven by elementary processes such as

$$
v_{\mu}+p \rightarrow \mu^{-}+\Delta^{++} \rightarrow \mu^{-}+p+\pi^{+},
$$

only requires minor changes [104]. In this case, the nucleon tensor $\mathcal{W}^{\lambda \mu}$ involves the matrix elements of the weak current describing the nucleon-resonance transitions. As a consequence, the structure functions-which can still be written in terms of phenomenological vector and axial-vector form factors-depend on both $q^{2}$ and $W^{2}$, the squared invariant mass of the hadronic final state. In addition, the energy conserving $\delta$-function in Eq.(53) is replaced by a Breit-Wigner factor according to

$$
\delta\left(W^{2}-m^{2}\right) \rightarrow \frac{M_{R} \Gamma_{R}}{\pi} \frac{1}{\left(W^{2}-M_{R}^{2}\right)^{2}+M_{R}^{2} \Gamma_{R}^{2}},
$$

where $M_{R}$ and $\Gamma_{R}$ denote the resonance mass and decay width, respectively [104, 113]. 


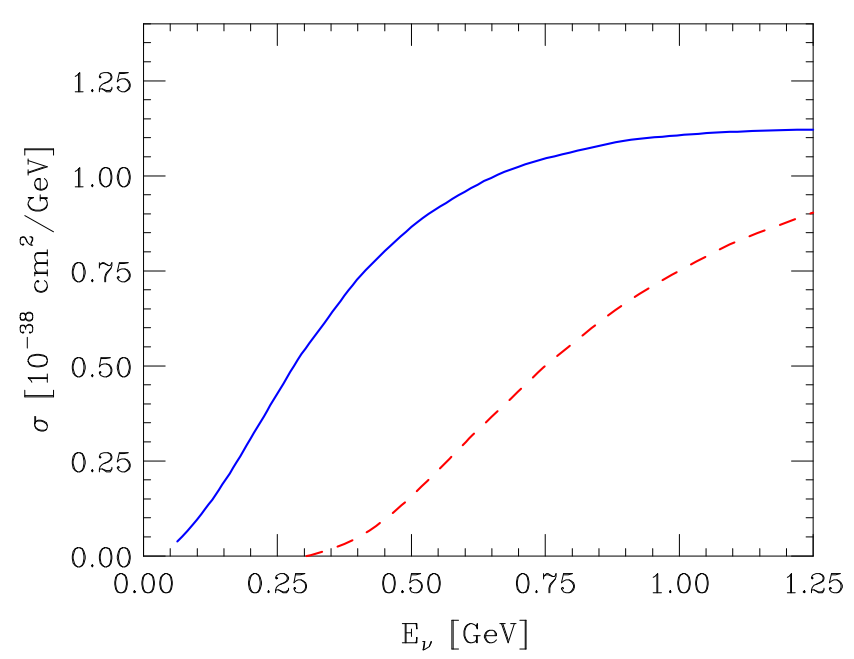

Figure 24: QE (solid line) and resonance production (dashed line) contributions to the total cross section of charged-current neutrino-nucleon interactions (adapted from Ref. [104]).

As an example, illustrating the relative weight of QE scattering and resonance producion, the $\mathrm{CC} \mathrm{QE}$ and resonance contributions to the total neutrino-nucleon cross section reported in Ref. [104] are shown in Fig. 24 as a function of neutrino energy. The resonance-production cross section has been obtained taking into account both the $P_{33}(1232) \Delta$ resonance and the three isospin $1 / 2$ states lying in the so-called second resonance region: $P_{11}(1440), D_{13}(1520)$ and $S_{11}(1535)$ [104]. It clearly appears that at beam energies $\gtrsim 1 \mathrm{GeV}, \mathrm{QE}$ scattering and resonance production turn out to be provide comparable contributions.

It must be kept in mind that he decay of the $\Delta$ resonance [see Eq. (60)] is a prominent mechanism leading to the appearance of pions, and that events in which the final state pion is absorbed in the target nucleus are a major background to QE processes. A detailed discussion of both coherent and incoherent pion production can be found in Refs. [114] and [115].

\subsection{Deep inelastic scattering}

From the observational point of view, the Deep Inelastic Scattering (DIS) regime corresponds to hadronic final states with more than one pion.

In principle, the three nucleon structure functions entering the definition of the IA nuclear cross section, Eqs. (21) and (22), may be obtained combining neutrino and antineutrino scattering cross sections. However, as the available structure functions have been extracted from nuclear cross sections (see, e.g., Ref. [116]), their use in ab initio theoretical studies aimed at identifying nuclear effects entails obvious conceptual difficulties.

An alternative approach, allowing to obtain the structure functions describing DIS on isolated nucleons, can be developed within the conceptual framework of the quark-parton model, exploiting the large database of accurate DIS data collected using charged lepton beams and hydrogen and deuteron targets (see, e.g., Ref. [117]). Within this scheme, the function $F_{2}^{\nu N}=\omega W_{2}$, where $\omega$ is the energy transfer and $W_{2}$ is the weak structure function of an isoscalar nucleon, defined as in Eq. (48), can be simply related to the corresponding structure function extracted from electron scattering data, $F_{2}^{e N}$, through ${ }^{5}$

$$
\begin{aligned}
F_{2}^{v N}\left(Q^{2}, x\right) & =x\left[u\left(Q^{2}, x\right)+\bar{u}\left(Q^{2}, x\right)+d\left(Q^{2}, x\right)+\bar{d}\left(Q^{2}, x\right)\right] \\
& =\frac{18}{5} F_{2}^{e N}\left(Q^{2}, x\right)
\end{aligned}
$$

where $x$ is the Bjorken scaling variable, while $q\left(Q^{2}, x\right)$ and $\bar{q}\left(Q^{2}, x\right)$, with $q=u, d$, denote the quark and antiquark distributions, respectively. In addition, the relation

$$
x \omega W_{3}\left(Q^{2}, x\right)=x F_{3}^{v N}=x\left[u_{\mathrm{v}}\left(Q^{2}, x\right)+d_{\mathrm{v}}\left(Q^{2}, x\right)\right],
$$

with $u_{\mathrm{v}}\left(Q^{2}, x\right)$ and $d_{\mathrm{v}}\left(Q^{2}, x\right)$ being the valence quark distributions, implies

$$
x F_{3}^{v N}=F_{2}^{e N}-2 x\left[\bar{u}\left(Q^{2}, x\right)+\bar{d}\left(Q^{2}, x\right)\right] .
$$

Using Eqs. (62)-(64) and the Callan-Gross relation, linking $F_{1}^{\nu N}=m W_{1}$ to $F_{2}^{\nu N}$, one can readily obtain all the weak structure functions from the existing parametrisations of the measured electromagnetic structure functions and of the antiquark distribution (see, e.g., Ref .[118]). Alternatively, the quark and antiquark distributions can be also used to obtain the structure function $F_{2}^{e N}\left(Q^{2}, x\right)$ from Eq.(62).

The above procedure rests on the tenet, underlying the IA scheme, that the elementary neutrino-nucleon interaction is not affected by the presence of the nuclear medium. While this assumption is strongly supported by electron-nucleus scattering data in the quasi elastic channel, showing no medium modifications of the nucleon vector form factors, analyses of neutrino DIS data are often carried out allowing for a medium modification of the nucleon structure functions [119, 120], or of the parton distributions entering their definitions [121].

The approach of Ref. [119, 120] makes use of a model of the nuclear spectral function, and includes a variety of medium effects, such as the $\pi$ - and $\rho$-meson cloud contributions and nuclear shadowing. On the other hand, Ref. [121] provides a parametrization of the nuclear parton distributions to order $\alpha_{s}-\alpha_{s}$ being the coupling constant of strong interactions-obtained from a fit to the measured nuclear cross sections.

\section{Interpretation of CC QE events}

The data set of CC QE events collected by the MiniBooNE collaboration [13] provides an unprecedented opportunity to carry out a systematic study of the double differential cross section of the process,

$$
v_{\mu}+{ }^{12} C \rightarrow \mu^{-}+X
$$

\footnotetext{
${ }^{5}$ For simplicity, here and in what follows we will neglect the contributions of $s$ - and $c$-quarks.
} 
averaged over the neutrino flux shown in Fig. 3.

As pointed out in the previous section, the CC QE neutrinonucleon process is described in terms of three form factors. The proton and neutron electromagnetic form factors, which have been precisely measured up to large values of $Q^{2}$ in electronproton and electron-deuteron scattering experiments, and the nucleon axial form factor $F_{A}$, parametrized in terms of the axial mass $m_{A}$ as in Eq. (59). The data analysis performed using the RFGM yields an axial mass $m_{A} \approx 1.35 \mathrm{GeV}$, significantly larger than that obtained from deuteron data [111, 112]. A large value of the axial mass, $m_{A} \approx 1.2 \mathrm{GeV}$, has been also obtained from the analysis of the CC QE neutrino-oxygen cross section carried out by the K2K collaboration [122], while the NOMAD collaboration reported the value $m_{A}=1.05 \mathrm{GeV}$-compatible with the world average of deuteron data-resulting from the analysis of CC QE neutrino- and antineutrino-carbon interactions at much larger beam energies $\left(\left\langle E_{v}\right\rangle \sim 26 \mathrm{GeV}\right)[123]$.

It would be tempting to interpret the value of $m_{A}$ reported by MiniBoonNE as an effective axial mass, modified by nuclear effects not included in the RFGM. However, theoretical studies carried out within the IA scheme with a realistic carbon spectral function-an approach providing a satisfactory account of the electron scattering cross section in the quasi elastic sector-fail to describe the flux averaged double differential cross section of Ref. [13]. This striking feature is illustrated in Figs. 25 and 26. Figure 25 shows a comparison between the electron scattering data data of Ref. [31] and the results obtained using the spectral function of Ref. [42], while in Fig. 26 the results obtained within the same scheme and setting $m_{A}=1.03 \mathrm{MeV}$ are compared to the flux averaged double differential CC QE cross section measured by the MiniBooNE collaboration, shown as a function of kinetic energy of the outgoing muon [124]. It is apparent that height, position and width of the QE peak measured in electron scattering, driven by the energy and momentum dependence of the spectral function, respectively, are well reproduced. On the other hand, the peaks exhibited by the neutrino cross sections are largely underestimated.

The authors of Ref. [124] argued that the differences observed comparing Fig. 25 to Fig. 26 are to be largely ascribed to the flux average involved in the determination of the neutrino cross section, leading to the appearance of contributions of reaction mechanisms not taken into account in the IA picture.

In MiniBooNE data analysis, an event is labeled as CC QE if no final state pions are detected in addition to the outgoing muon. The adjective elastic is therefore intended as alternative to inelastic, as it should, and event selection is performed in a model independent fashion.

The simplest reaction mechanism compatible with the above qualification is single nucleon knockout, induced by the onenucleon contributions to the nuclear current [see Eq. (4)]. As pointed out above, in the absence of NN correlations the spectator $(A-1)$-particle system is left in a bound state, and the final state, consisting of the knocked out nucleon and the recoiling residual nucleus, is a $1 \mathrm{p} 1 \mathrm{~h}$ state.

It has been suggested that the observed excess of $\mathrm{CC} \mathrm{QE}$ cross section may be traced back to the occurrence of events with $2 \mathrm{p} 2 \mathrm{~h}$ final states, discussed in Section 3.5 [125, 126]. Ac-

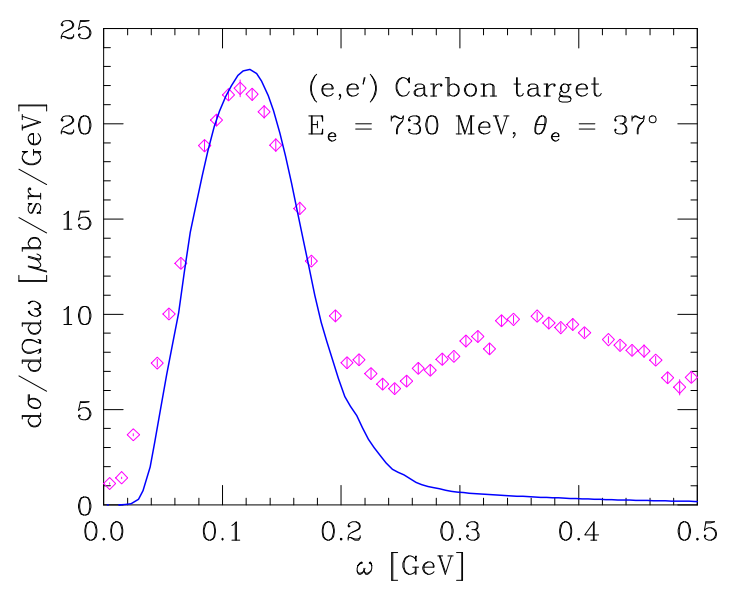

Figure 25: Inclusive electron-carbon cross section at beam energy $E_{e}=730$ $\mathrm{MeV}$ and electron scattering angle $\theta_{e}=37^{\circ}$, plotted as a function of the energy loss $\omega$ [124]. The solid line shows the results obtained using the spectral function formalism. The data points are taken from Ref. [31].

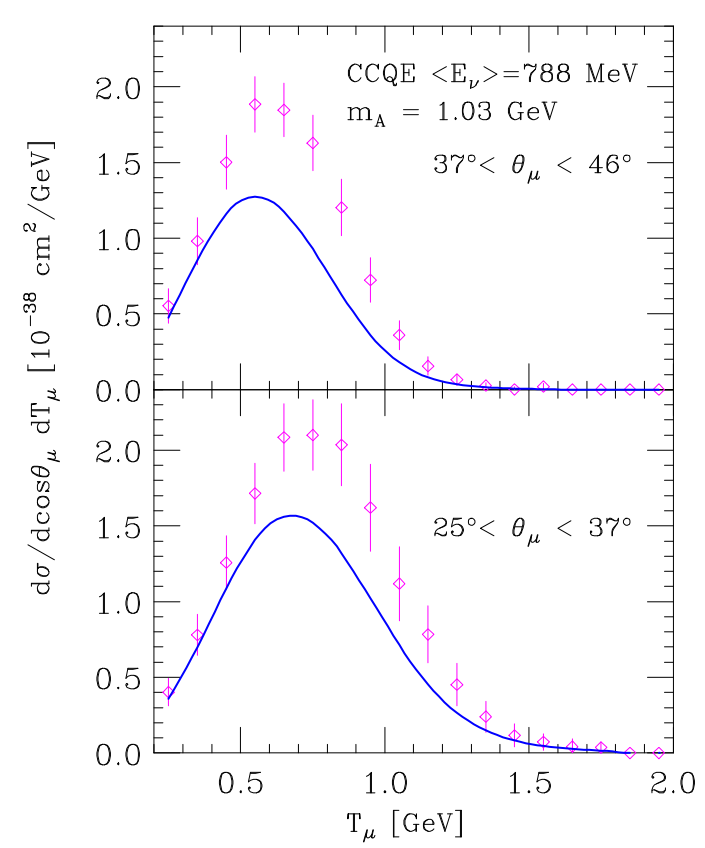

Figure 26: Flux averaged double differential CC QE cross section measured by the MiniBooNE collaboration [13], shown as a function of the kinetic energy of the outgoing muon [124]. The upper and lower panels correspond to different values of the muon scattering angle. Theoretical results have been obtained using the same spectral functions and vector form factors employed in the calculation of the electron scattering cross section of Fig. 25, and a dipole parametrization of the axial form factor with $m_{A}=1.03 \mathrm{GeV}$.

cording to the above classification, these events cannot be distinguished from those with $1 \mathrm{p} 1 \mathrm{~h}$ final states . Therefore, they are often referred to as CC QE-like. The excitation of $2 \mathrm{p} 2 \mathrm{~h}$ final states at higher energies, up to $10 \mathrm{GeV}$, has been also recently discussed by the authors of Ref. [127].

The role of neutrino interactions leading to the appearance of multi-particle-multi-hole final states was first pointed out by Marteau and collaborators [128], who also took into account 
the effect of long range correlations within the RPA scheme. The underlying nuclear model, originally developed in the early 1990s to describe $\left({ }^{3} \mathrm{He},{ }^{3} \mathrm{H}\right)$ charge exchange reactions [129], included perturbative $\pi$ - and $\rho$-exchange interactions, supplemented with contact terms providing an approximate description of short-range dynamics [130].

The authors of Refs. [125] and [126] carried out extensive calculations of the CC QE neutrino-carbon cross section, averaged over the MiniBooNE flux, taking into account the effects of the two-nucleon current as well as collective nuclear excitations, which are expected to play a role at low momentum transfer. As an example, in Fig. 27 the results of these approaches, obtained using a value of the axial mass consistent with the one extracted from deuteron data, are compared to the MiniBooNE muon energy spectrum at muon scattering angle $\theta_{\mu}$ such that $0.8 \leq \cos \theta_{\mu} \leq 0.9$. After inclusion of the reaction mechanisms beyond single-nucleon knock out, both schemes turn out to provide a significantly improved description of the measured cross section. Note that in the left panel the data have been rescaled by a factor 0.9 , to allow for a $10 \%$ normalisation uncertainty [126].

The contribution of interactions involving the two-nucleon current has been also recently investigated by the authors of Ref. [131], who generalised the SuSav2-MEC approach-briefly outlined in Section 3-to the case of neutrino interactions. The results of this study are illustrated in Fig. 28.

The authors of Ref. [131] carried out a detailed study of the cross section associated with processes involving MEC. The results of this analysis show that in neutrino interactions the contribution of the longitudinal channel is more significant than in electromagnetic interactions, and arises mainly from the the axial-vector current.

The emerging picture strongly suggests that the inclusion of contributions other than single-nucleon knock out is needed to bring theoretical calculations into agreement with the measured cross sections. However, it must be pointed out that long range RPA correlations, while providing significant contributions to the cross sections reported in Refs. [125] and [126], do not appear to be needed within the SuSav2-MEC approach of Ref. [131].

\section{Implementation of nuclear dynamics in Monte Carlo simulations}

As pointed out in the previous Sections, the generalisation of the theoretical description of electron-nucleus scattering to the case of neutrino interactions does not involve severe conceptual difficulties. However, while significant progress has been made in the understanding of the different reaction mechanisms contributing to the signals detected by neutrino experiments, the implementation of state-of-the-art models in the existing Monte Carlo generators has been lagging behind. Among the many reasons of this state of affairs, one of the most prominent is that in neutrino oscillation experiments event generators are used to predict how the signal and background events will appear in the neutrino detector. Therefore, each generator is expected to simulate all relevant interactions, and each simulation has to cover all possible kinematical regions. Additional complications arise from the requirement of being able to describe the variety of nuclei used for detection.

Most available neutrino event generators rely on the RFGM for the treatment of the nuclear ground state. Recently, an improved implementation of the spectral function approach [23, 42, 132], based on the formalism described in Section 3.2, has been included in the GENIE event generator [133, 134]. The event generators NUWRO and NEUT also feature a spectral function implementation, aimed at improving the description of the nucleon energy and momentum distribution $[135,136]$.

As a first step, the authors of Ref. [134] focused on the CC QE channel, which accounts for a large fraction of the detected signal in many neutrino oscillation experiments. As an example, Fig. 29 presents the double differential cross section of the process

$$
v_{\mu}+{ }^{12} \mathrm{C} \rightarrow \mu^{-}+X,
$$

in the QE channel, at neutrino energy $E_{v}=1 \mathrm{GeV}$ and muon scattering angle $\theta_{\mu}=30 \mathrm{deg}$, plotted as a function of the lepton energy loss $\omega$. The calculation has been carried out using the carbon spectral function of Ref. [42]. In order to illustrate the size of the axial-vector contributions, the result of the full calculation is compared to that obtained setting $F_{A}\left(Q^{2}\right)=0$.

To carry out the simulation following the scheme outlined above, few new modules were developed, and few modules from the official GENIE release 2.8.0 were modified [134]. From now on, we will refer to them as GENIE 2.8.0 $+v T$. By analogy with Eq. (21), the QE neutrino-nucleus cross section at beam energy $E_{v}$ can be written in the target rest frame as

$$
\begin{gathered}
\left(\frac{d^{2} \sigma_{v A}}{d E_{\mu} d \Omega_{\mu}}\right)=\int d^{3} k d E\left(\frac{d^{2} \sigma_{v N}}{d E_{\mu} d \Omega_{\mu}}\right) P(|\mathbf{k}|, E) \\
\times \delta\left(\omega+M_{A}-\sqrt{|\mathbf{k}+\mathbf{q}|^{2}+m^{2}}-E_{A-1}\right) .
\end{gathered}
$$

The integrations can be carried out using the Monte Carlo method, yielding

$$
\left(\frac{d^{2} \sigma_{v A}}{d E_{\mu} d \Omega_{\mu}}\right) \approx \frac{1}{N} \sum_{n=1}^{N} G\left(E_{v}, E_{\mu}, \cos \theta_{\mu} ;\left\{k, E, \cos \theta_{N}\right\}_{n}\right),
$$

where $E_{\mu}$ and $\theta_{\mu}$ denote the muon energy and scattering angle, respectively, $\theta_{N}$ is the polar angle specifying the direction of the nucleon momentum, $\mathbf{k}$, and

$$
\begin{aligned}
G\left(E_{\nu},\right. & \left.E_{\mu}, \cos \theta_{\mu} ; k, E, \cos \theta_{N}\right)=\left(\frac{d^{2} \sigma_{v N}}{d E_{\mu} d \Omega_{\mu}}\right) \\
& \times \delta\left(\omega+M_{A}-\sqrt{|\mathbf{k}+\mathbf{q}|^{2}+m^{2}}-E_{A-1}\right) .
\end{aligned}
$$

In Eq. (68), $\left\{k, E, \cos \theta_{N}\right\}_{n}$ denotes the set of kinematical variables of the struck nucleon. Momentum and energy, $k$ and $E$, are sampled from the probability distribution $F(k, E)=$ $4 \pi|\mathbf{k}|^{2} P(|\mathbf{k}|, E)$, while $\cos \theta_{N}$ is assumed to be uniformly distributed in the range $[-1,1]$.

In its release 2.8.0 $+v T$, the GENIE event generator provides a simulation of CCQE neutrino interactions based on two different description of the nuclear ground state: the RFGM and 

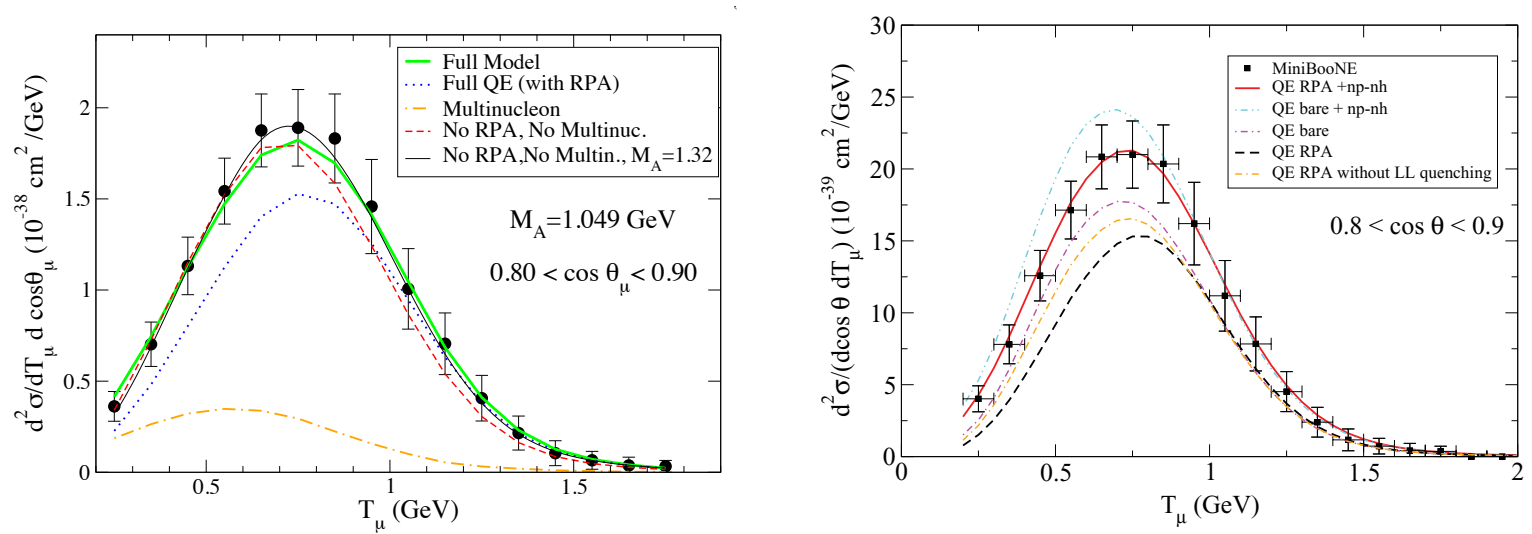

Figure 27: Comparison between the flux averaged muon energy spectrum at muon scattering angle $\theta_{\mu}$ corresponding to $0.8 \leq$ cos $\theta_{\mu} \leq 0.9$, measured by the MiniBooNE collaboration [13], and the theoretical results of Ref. [125] (thick solid line of the right panel) and Ref. [126] (thick solid line of the left panel). Both theoretical calculations have been carried out using a value of the axial mass consistent with those reported in Refs.[111, 112]. Note that the data in left panel have been rescaled by a factor 0.9 .

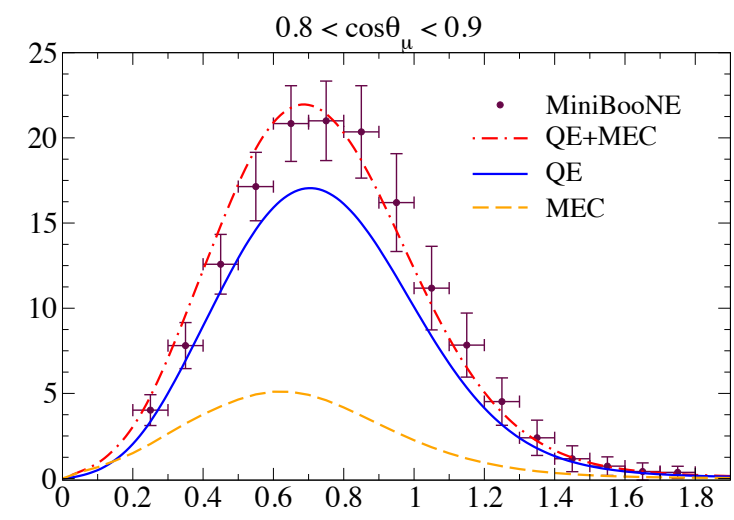

Figure 28: Comparison between the flux averaged muon energy spectra measured by the MiniBooNE collaboration at muon scattering angle $\theta_{\mu}$ corresponding to $0.8 \leq \cos \theta_{\mu} \leq 0.9$ [13] and the results of the SuSav2-MEC approach [131].

the spectral function approach. In addition to the CC QE channel, both nuclear models can be used to simulate interactions leading to different hadronic final states, such as resonance production and decay, pion production and deep-inelastic scattering. A detailed description of the treatment of these processes can be found in the literature [133, 134, 137].

As an example of the GENIE 2.8.0 $+v T$ results, in Fig. 30 we show the electron scattering cross sections obtained using the LDA carbon spectral function of Ref. [42] and the calcium and argon spectral functions obtained from the simplified approach of Ref. [132]. In order to allow for a consistent comparison with the data-that were not corrected to remove the effects of the FSI - the results of the simulations are presented with and without inclusion of FSI [138].

The agreement appears to be quite satisfactory, the differences being largely ascribable to numerical accuracy. The effects of FSI discussed in Section 3.4-that is, a shift of the energy loss distribution and a redistribution of the strength from

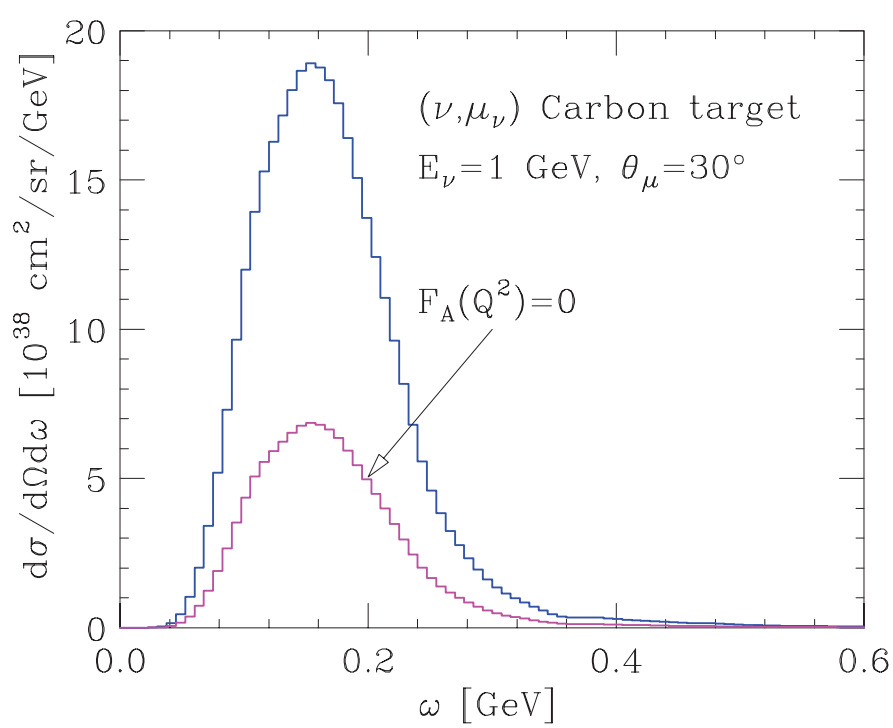

Figure 29: Double differential cross section of the process $v_{\mu}+{ }^{12} \mathrm{C} \rightarrow \mu^{-}+X$ in the QE channel, obtained using the spectral function of Ref. [42]. The two histograms show the results of the full calculation and those obtained setting $F_{A}\left(Q^{2}\right)=0$ [134].

the quasi elastic peak to the tails - can be clearly observed in the GENIE 2.8.0 $+v T$ results, and appear to be more pronounced in the case of heavier targets. Note that the tail of the inelastic contributions extending into the region of the QE peak are quite small (see, e.g., Ref. [23]), and their inclusion does not appreciably affect the emerging scenario.

A consistent implementation of the IA requires a careful consideration of the $Q^{2}$ selection, taking into account the fact that, while the tensor $L^{\lambda \mu}$ of Eq. (46) is determined from lepton kinematical variables only, the nucleon tensor depends on the initial nucleon momentum, $\mathbf{k}$, and $\widetilde{q} \equiv(\widetilde{\omega}, \mathbf{q})$, which in turn depends on the removal energy $E$ through its time component $\widetilde{\omega}$, defined by Eq. (20). The energy transfer at the elementary interaction vergex is $\widetilde{\omega}<\omega$, while the difference $\delta \omega=\omega-\widetilde{\omega}$ provides 


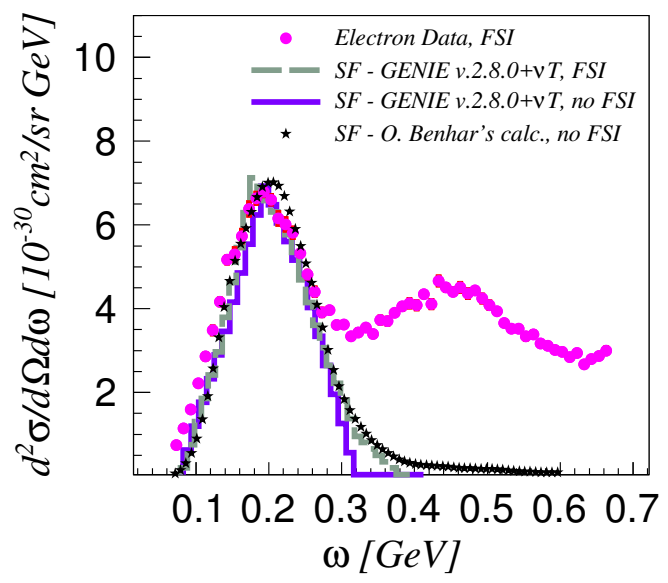

(a) $e+\mathrm{C} \rightarrow e^{\prime}+X, E_{e}=0.961 \mathrm{GeV}, \theta_{e}=37.5 \mathrm{deg}$

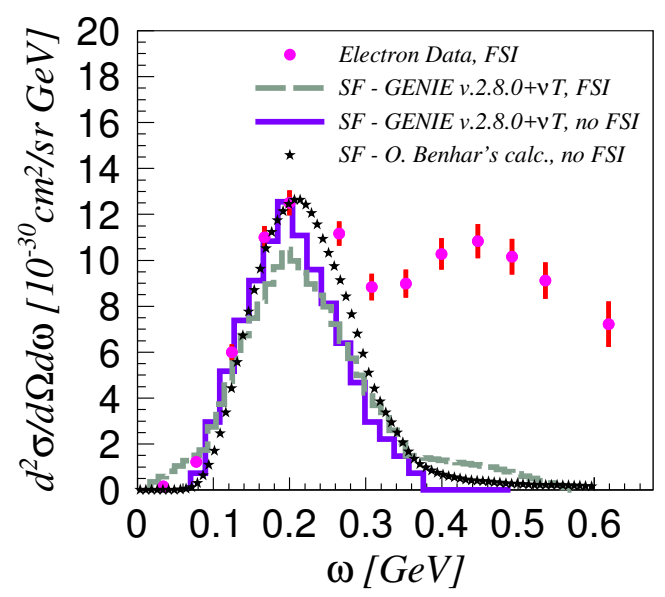

(c) $e+\mathrm{Ca} \rightarrow e^{\prime}+X, E_{e}=0.841 \mathrm{GeV}, \theta_{e}=45.5 \mathrm{deg}$

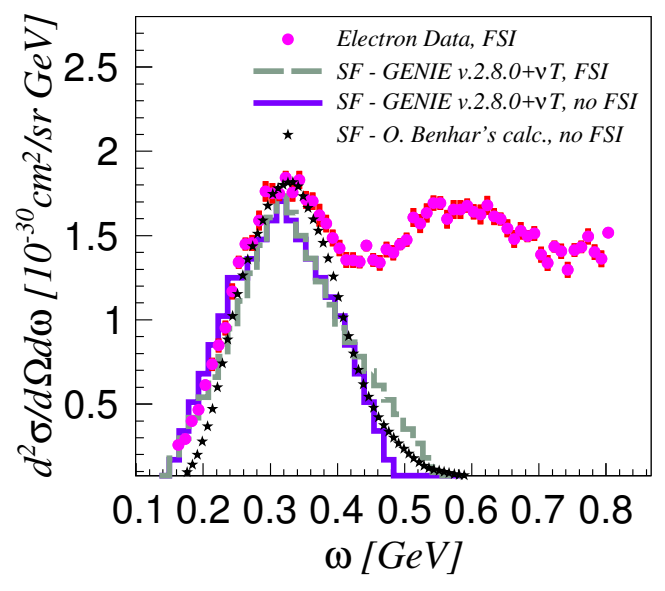

(b) $e+\mathrm{C} \rightarrow e^{\prime}+X, E_{e}=1.299 \mathrm{GeV}, \theta_{e}=37.5 \mathrm{deg}$

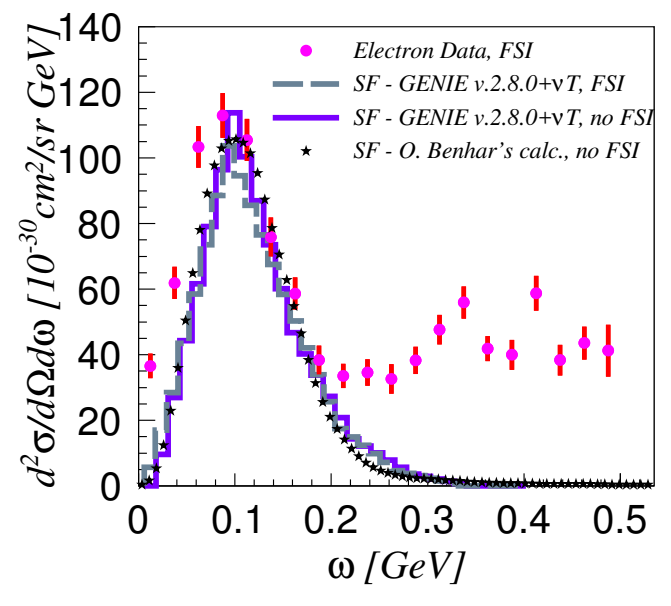

(d) $e+\mathrm{Ar} \rightarrow e^{\prime}+X, E_{e}=0.700 \mathrm{GeV}, \theta_{e}=32 \mathrm{deg}$

Figure 30: Double differential electron-nucleus cross section in the quasielastic channel. The curves labeled SF have been obtained using Eq. (68) and the model spectral functions of Refs. [42] (for carbon) and [132] (for calcium and argon) [134]. The data are taken from Refs. [32] (for carbon), [139] (for calcium), and [140] (for argon). 
a measure of the energy transfer to the spectator system. The details of the $Q^{2}$-selection procedure can be found in the paper of Jen and collaborators [134].

Unfortunately, the full description of the complex dynamics of nuclear scattering processes requires the use of more variables and observables than just the four-momenta of the target nucleon, the outgoing leptons and all the produced hadrons and gammas. Hence, the comparison between the observed neutrino interactions and the predictions of nuclear models needs to be done with great care. Because neutrino event generators are usually tuned to existing data, the implementation of a new model in an existing framework may result in making the comparisons more difficult. Individual nuclear models should be evaluated considering their limitations and the kinematical region in which they are expected to be valid, and must be compared to a set of external data, when available. An example of this procedure is given in Fig. 31, showing a comparison between two different implementations of the spectral function approach and the results of a $\left(e, e^{\prime}\right)$ measurement [panel (a)], as well as the corresponding predictions for the outgoing muon distribution in neutrino interactions [panel (b)]. As an external data set, the electron scattering cross sections turn out to provide an excellent validation tool.

\section{Dependence of oscillation parameters on the description of nuclear effects}

The discovery of neutrino oscillation has been one of the major results of particle physics in the past two decades. As a consequence, we are witnessing a massive global experimental effort, in the form of ongoing and planned long- and shortbaseline oscillation experiments. At short baselines, of order $1 \mathrm{~km}$ or less, the goal is to conclusively test the hypothesis of an eV-scale sterile neutrino (see, e.g., Ref. [141]), whereas at long baselines, of several $100 \mathrm{~km}$ or more, the goal is to measure the leptonic $\mathrm{CP}$ phase, and to ultimately test the validity of the three neutrino oscillation paradigm. All of these experiments will use detectors made of nuclei with masses in the range $A=12-56$. Therefore, a precise understanding of the electro-weak response of these nuclei to both neutrino and antineutrino interactions is necessary. The relevant neutrino energies range from a few hundreds $\mathrm{MeV}$ up to approximately $10 \mathrm{GeV}$, thus fully covering the quasi-elastics region, but also largely extending into the domain of resonance production and DIS. As explained in the previous Sections, this is a considerable challenge for theory. From an experimental view point, the problem is severely compounded by the limitations of currently used neutrino sources and the fact that in the detector different underlying events yield identical signatures.

In this Section, we establish a connection between the oscillation physics measurements and the required level of precision in the understanding of neutrino-nucleus interactions. We also demonstrate, using a number of specific examples which have been studied in detail in the literature, the impact the current level of uncertainty surrounding neutrino-nucleus cross sections has on the ability to correctly interpret experimental results. Regretfully, despite these efforts important special

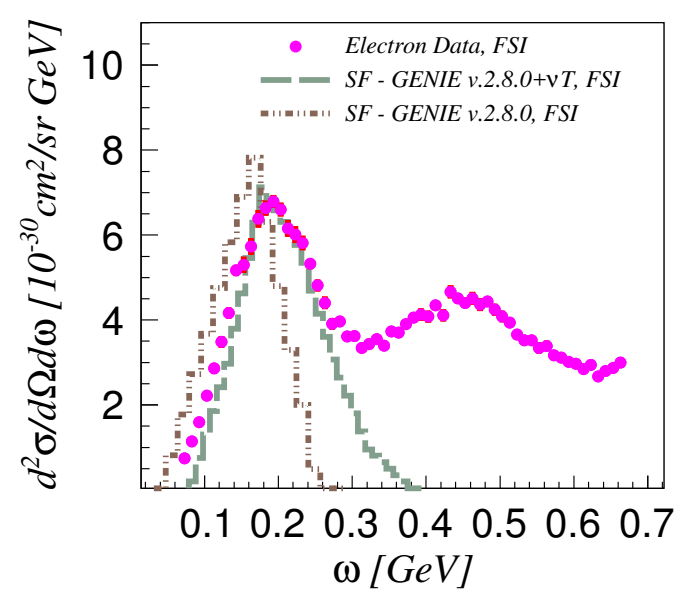

(a) Inclusive electron-carbon cross section at beam energy $\mathrm{E}_{e}=0.961 \mathrm{GeV}$ and scattering angle $\theta_{e}=37.5 \mathrm{deg}$.

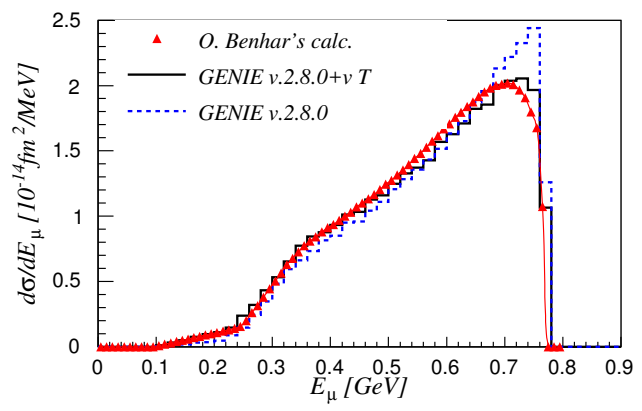

(b) Outgoing muon energy distribution corresponding to muon neutrinos of $0.8 \mathrm{GeV}$ interacting with oxygen.

Figure 31: Comparison between different implementation of the spectral function model [134]. In panel (a) the results obtained from GENIE 2.8.0 and GENIE 2.8.0 $+v T$ are displayed using dots and the dot-dash line, respectively, and compared to the data of Ref. [31]. In panel (b) the GENIE 2.8.0 and GENIE 2.8.0 $+T$ results are represented by the dotted and solid line, while the theoretical results of Benhar and collaborators [23] (labeled O. Benhar's calc.) are shown by triangles. The effect of Pauli blocking is included in all three calculations, while FSI are not taken into account.

cases - notably CP violation searches using liquid argon detectors-have not yet been studied in sufficient detail to make reliable, quantitative statements.

Before embarking on detailed case studies, we first describe the basic problem based on a number of approximations and give some examples based on these approximations. We analyze in detail the appearance $v_{\mu} \rightarrow v_{e}$ and disappearance $v_{\mu} \rightarrow v_{\mu}$ channels in the T2K experiment, to show that such uncertainties are relevant also in the extraction of the atmospheric parameters. This analysis is performed comparing the RFGM, the approach based on a realistic spectral function and two different implementations of the RPA scheme, as well as differ- 
ent event generators for the disappearance analysis. Here, we limit ourselves to the quasi-elastic regime, where the neutrino cross sections can be evaluated with less uncertainties.

It is worthwhile to point out that many of the problems arise from the need to reconstruct the neutrino energy precisely, since it is the neutrino energy which enters the oscillation probability. At the rate level many quantitative studies have been performed. The uncertainties in the energy reconstruction resulting from our lack of an understanding of the underlying micro-physics are very hard to analyze quantitatively for two reasons: First, this relies on the ability to model exclusive cross sections, since the energy distribution of secondary particles produced in the neutrino event has to be quantified. There is very little data to support this modeling and the sparse data we have does not fit with any existing model. It is therefore, very difficult to establish the range of plausible variations of energy dependencies. Second, neutrino energy reconstruction also depends on fine details of detector performance and in particular, for liquid argon detectors, there is not yet a good quantitative understanding of detector performance. As a corollary, there clearly is awareness that energy reconstruction is profoundly affected by exclusive cross sections and their uncertainties, but there a few quantitative studies and those which exist are highly simplified, we discuss some examples in section 7.4.

Finally, we point out necessary steps towards a full quantitative understanding of the relation between cross section uncertainties and oscillation physics measurements for the next generation of long-baseline experiments.

\subsection{The problem at rate-level}

Neutrino physics is on its way to become precision science, and this brings new challenges for future experiments. The next 10-20 years will be centered on a long-baseline neutrino oscillation program using conventional neutrino beams obtained from pion decay-in-flight. This technique of making neutrino beams has been used for many decades: an intense proton beam impacts on a thick target producing mostly pions, but also other mesons. The pions are focused using a magnetic horn, and the polarity of the magnetic field allows to select predominantly one charge-sign of the pion, which implies the ability to make neutrino and antineutrino beams. It is important to note, that the resulting fluxes and purities of neutrino and antineutrino beams are not related in a meaningful way. Therefore, the neutrino and antineutrino beams derived from the same target and horn configuration are essentially independent experiments. The next generation of beams will exceed the $1 \mathrm{MW}$ level of power on target, which represent a major advance in engineering and accelerator physics. Hence, these beams are dubbed superbeams. It is a very difficult task to determine the resulting neutrino flux, energy spectrum and flavor composition purely from data on meson production in thick targets, beam parameters and horn configuration. Currently, the state of the art in controlling beam systematic uncertainties is represented by the MINOS [142] and MINERvA [143] experiments. Much of the information used, in both cases, comes from the ability to change the position of the target with respect to the horn system. This ability most likely will not exist for beams with target powers in excess of
$1 \mathrm{MW}$, due to the resulting very harsh operating conditions in close proximity to the target. Therefore, it appears reasonable to assume that the understanding of the beam at the level of roughly 5\%, demonstrated by MINOS and MINERvA, represents the best case for future experiments as well. More precise neutrino beams require a different technological approach. For instance, muon decay offers the possibility to obtain highintensity $v_{\mu}$ and $v_{e}$ beams with beams systematics well below $1 \%$. This concept is know as neutrino factory [144], and a lowenergy entry-level version is know as $v$ STORM [145], which would allow, among other applications, a very precise and accurate measurement of neutrino cross sections.

As explained in the previous Sections, the current understanding of cross sections-where understanding implies the ability to describe actual experimental data-is generally at the $10 \%$ level, and neutrino beams are known at the 5\% level. Thus, the question is: what level of accuracy is needed for future neutrino oscillation measurements? One of the main goals of the future neutrino program is a measurement of the leptonic $\mathrm{CP}$ phase, $\delta$. It also happens that this measurement puts the most stringent demands on the overall accuracy, since it involves both neutrinos and antineutrinos and the relevant oscillation probability $P\left(v_{\mu} \rightarrow v_{\mu}\right)$ depends on all three mixing angles and both $\delta m^{2}$ in leading order, see $e . g$. Ref. [146]. The resulting requirement on systematic uncertainties is closely tied to the required level of accuracy in the determination of the CP phase. There is no a priori physics arguments which would argue for an error of $x$ degrees in the measurement of $\delta$, like for instance in the case of QED, where new effects clearly appear as powers of the fine structure constant. Arguments can be made based on certain neutrino flavor models, like sum rules [147], but these arguments remain model-dependent. Another line of argumentation, is based on the recognition that $\mathrm{CP}$ violation is not well understood in the Standard Model, in the sense that the QCD Lagrangian would allow for CP violation but is CP conserving to a very high degree of accuracy, the so-called strong CP problem - for a brief introduction see Ref. [148]—whereas mixing in the quark sector shows large $\mathrm{CP}$ violation. Framed in this way, the question of how large $\mathrm{CP}$ violation in the lepton sector is, if it exists at all, becomes very relevant. The ability of an experiment to discover CP violation depends on the one hand on the existence of CP violation, in this case $\delta \neq 0, \pi$, and on the ability to distinguish the measured value for $\delta$ from the $\mathrm{CP}$ conserving cases 0 and $\pi$. A reasonable goal for an experiment could be the ability to discover CP violation at $3 \sigma$ confidence level for $75 \%$ of all CP phases. This goal was for instance adopted recently in the U.S. [149].

A good proxy for the ability to measure the $\mathrm{CP}$ phase is given by the CP asymmetry, $A$, defined as

$$
A=\frac{\langle P\rangle-\langle\bar{P}\rangle}{\langle P\rangle+\langle\bar{P}\rangle},
$$

where $\langle P\rangle$ is the energy averaged oscillation probability for $v_{\mu} \rightarrow v_{e}$ and $\bar{P}$ is the corresponding quantity for antineutrinos. The energy average is taken over the range defined by having one half of the peak probability around the first oscillation maximum. In vacuo, $A$ is proportional to $\sin \delta$ and thus the errors on 


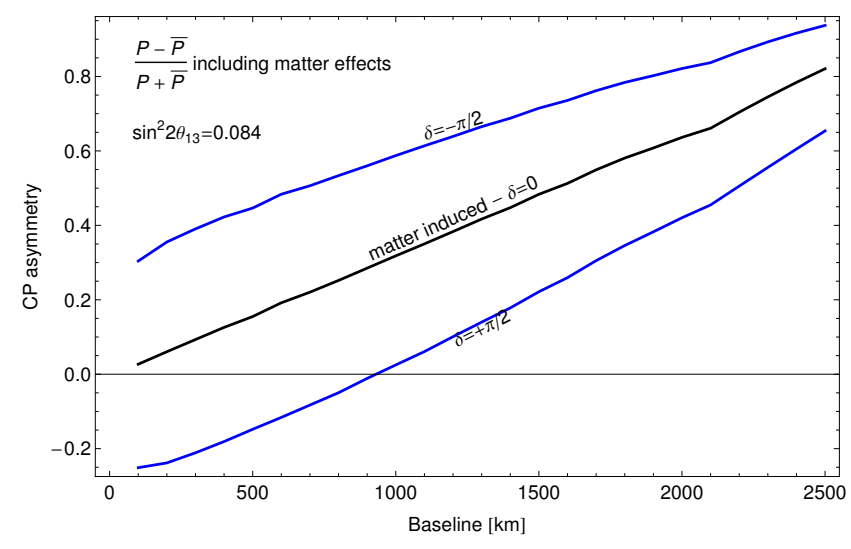

Figure 32: Value of the $\mathrm{CP}$ asymmetry $A$, for different choices of $\delta$, as a function of the baseline.

$A$ and $\delta$ are very similar for $\delta=0, \pi$. $A$ also will receive contributions from matter effects, which manifestly break CP invariance, as shown by the line labeled $\delta=0$ in Fig. 32. Here, the value of the asymmetry as a function of the baseline is shown for oscillation parameters from a recent global fit [150]. From this figure it is evident, that the maximal CP induced asymmetry is $\pm 25 \%$ for baselines of about $1,500 \mathrm{~km}$ on top a of a similar contribution from matter effects. For $75 \%$ of all CP phases the genuine $\mathrm{CP}$ asymmetry can become as small as 5\%, which at $3 \sigma$ translates into about $1.5 \%$ required accuracy. Further assuming, that the contributions from statistics and systematics should be about equal, this translates into a systematics requirement of $1 \%$. Obviously, for different CP violation discovery goals, this value will change correspondingly.

In practice experiments do not measure oscillation probabilities but event rate distributions, $R\left(E_{\mathrm{vis}}\right)$, as a function of the visible energy, $E_{\mathrm{vis}}$,

$$
\begin{aligned}
& R_{\beta}^{\alpha}\left(E_{\mathrm{vis}}\right)= \\
& N \int d E \Phi_{\alpha}(E) \sigma_{\beta}\left(E, E_{\mathrm{vis}}\right) \epsilon_{\beta}(E) P\left(v_{\alpha} \rightarrow v_{\beta}, E\right),
\end{aligned}
$$

where $N$ is a normalization factor, $\Phi_{\alpha}(E)$ is the neutrino flux as a function of the neutrino energy, $E$ and $P\left(v_{\alpha} \rightarrow v_{\beta}, E\right)$ is the oscillation probability as function of the neutrino energy. The differential cross section $\sigma_{\beta}\left(E, E_{\mathrm{vis}}\right)$ describes the probability that a neutrino of energy $E$ produces a distribution of visible energies $E_{\text {vis }}$ in the detector. Finally, $\epsilon_{\beta}(E)$ is the detection efficiency, and since it appears always in combination with $\sigma$ one can define the effective cross section, $\tilde{\sigma}_{\beta}:=\sigma_{\beta} \epsilon_{\beta}$. Note, that for this qualitative discussion we neglect any effects from the detector energy response and reconstruction efficiencies, which in general will add another level of complexity in terms of the relation between visible and reconstructed energy. Neglecting all energy dependencies of the flux, $\Phi_{\alpha}$, the effective cross section $\tilde{\sigma}$, Eq. (71) describes how the total event rate depends on the average oscillation probability. Even in this simplifying limit, there is no reason to assume that any of the quantities on the right hand side of Eq. (71) will be known better than 5-10\%. Also, ratios for neutrino and antineutrinos as well as flavor ratios are not a priori constrained to a better level of accuracy.
At quark level lepton universality prevails, but since we deal with nuclei and do not resolve the $Q^{2}$ of the interaction, nontrivial flavor effects are found especially at low energies [151]. Finally, the ratio of detection efficiencies $\epsilon_{e} / \epsilon_{\mu}$ has to be determined experimentally.

The problem of performing accurate measurements of oscillation parameters in the presence of significant cross section and/or flux uncertainties has been encountered before. A practical solution in many cases has been to use a near detector to measure the unoscillated event rate and exploit the fact that in the comparison of near and far detector data many uncertainties cancel. This method was used with great success in the Daya Bay experiment to measure $\theta_{13}$ [7]. For this cancellation to occur efficiently it is essential that near and far detectors have as close as possible an identical response to the neutrino signal. Any differences have to be understood with great precision. There are many potential sources for different near and far detector response functions, e.g. geometric acceptance or different background levels. Using the simplifying assumption that near and far detectors have identical response, for the total event rate ratio one obtains

$$
\begin{aligned}
\frac{R_{\alpha}^{\alpha}(\text { far }) L^{2}}{R_{\alpha}^{\alpha}(\text { near })} & =\frac{N_{\text {far }} \Phi_{\alpha} \tilde{\sigma}_{\alpha} P\left(v_{\alpha} \rightarrow v_{\alpha}\right)}{N_{\text {near }} \Phi_{\alpha} \tilde{\sigma}_{\alpha} 1} \\
& =\frac{N_{\text {far }}}{N_{\text {near }}} P\left(v_{\alpha} \rightarrow v_{\alpha}\right)
\end{aligned}
$$

In Daya Bay the conditions for this cancellation to occur were, by design, nearly ideal: the near and far detectors have the same size, they are made from the same materials, the reactors appear as point sources to both, the inverse beta-decay cross section is independently known, and the initial and final flavor is the same.

In extrapolating from the Daya Bay experience to future, long-baseline experiments a number of factors should be considered. First, to make the neutrino source, which in reality is the whole length of the decay pipe, point-like in the near detector, a not-so-near near detector is required. For baselines longer than $1,000 \mathrm{~km}$ the required tunnelling is likely to be prohibitively expensive. Second, the enormous size of the far detector renders an equally sized near detector unfeasible, and thus the detectors cannot have identical response. Third, the beam energy spread in a neutrino beam is large enough that a wide variety of interaction mechanisms will contribute to the signal, and thus the energy dependence of the near/far ratio can no longer be neglected. For a disappearance measurement, MINOS can serve as a benchmark of how well a near/far comparison does reduce systematic errors [152].

In an appearance measurement final and initial neutrino flavors are different, which will lead to an additional term in Eq. (72) of the form $\tilde{\sigma}_{\beta} / \tilde{\sigma}_{\alpha}$. Measuring $\tilde{\sigma}_{\beta}$ in a beam of purely flavor $\alpha$ is impossible. The small component of $v_{e}$ present in the beam is overall even less well known than the primary beam flux and the relative smallness of the $v_{e}$ component will result in reduced statistics in the near detector. Recently, the T2K Collaboration presented a result on the $v_{e}$ cross section in a predominantly $v_{\mu}$ beam [153]. The total systematic error is about $16 \%$, mostly originating from the beam flux uncertainty and the de- 


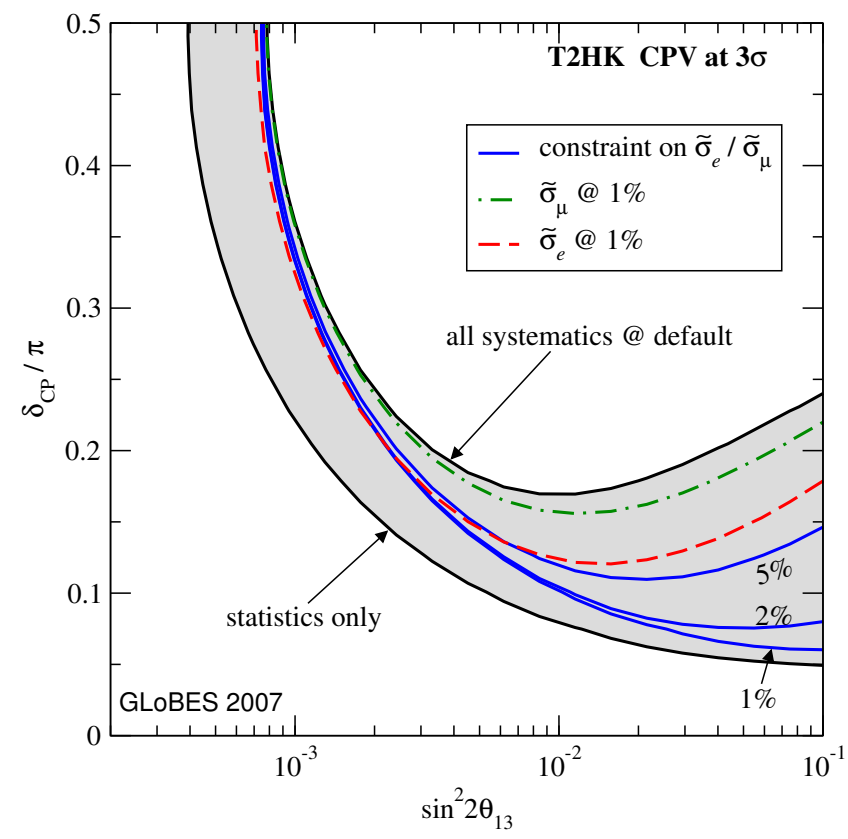

Figure 33: CP violation sensitivity at $3 \sigma$ level for a certain choice of systematical errors and for statistical errors only (curves delimiting the shaded region). We show also the sensitivity if certain constraints on the product of cross sections times efficiencies $\tilde{\sigma}$ are available: $1 \%$ accuracies on $\tilde{\sigma}_{\mu}$ and $\tilde{\sigma}_{e}$ for neutrinos and antineutrinos, and 5\%,2\%,1\% accuracies on the ratios $\tilde{\sigma}_{\mu} / \tilde{\sigma}_{e}$ for neutrinos and antineutrinos. Figure and caption adapted from Ref. [154].

tector response. There are no obvious methods to improve this situation and it stands to reason that for antineutrinos the situation will be worse. On general grounds, the ability to measure a cross section with much better accuracy than the accuracy at which the beam flux is known appears doubtful.

A quantitative analysis along these lines, based on only energy-independent implementations of uncertainties has been presented in Ref. [154], where a T2HK-like experiment was used to illustrate the impact of more than 20 potential sources of systematical uncertainties. This analysis, for the first time, explicitly included an idealized near detector. The main result for the sensitivity to measure CP violation-defined as the ability to exclude the CP conserving values of $\delta$ at the given confidence level-is shown in Fig. 33. It is apparent, that a tight constraint of $1 \%$ on the ratio of $\tilde{\sigma}_{e} / \tilde{\sigma}_{\mu}$ is required to restore the statistics-only result at large $\sin ^{2} 2 \theta_{13}$. A corollary from this work is that even a perfect near detector is not a panacea.

The analysis presented by the authors of Ref. [154] relied on a significant number of simplifying assumptions and is, in the present context, not so much important for its quantitative results, but it conceptually helps to frame the problem. It has been extended to a wide range of different experiments [155], without however improving on the underlying assumptions. In comparison of various different experiments, it turns out that experiments which rely on a relatively narrow beam spectrum and operate at energies below $1 \mathrm{GeV}$, like $\mathrm{T} 2 \mathrm{HK}$, are particularly sensitive to uncertainties on flavor ratios. On the other hand, experiments which employ a wide beam spectrum at multi-GeV energies, like LBNE, are much less affected by these rate-only uncertainties. The implementation of cross section uncertainties of Refs. [154] and [155] is naive at best, and the remainder of this section is devoted to more sophisticated case studies based either on specific cross section models or event generators. The spirit of these examples, is that in order to estimate an unknown (and uncomputable) theory error, an evaluation of the spread between different theory calculation is performed, and this spread somehow is indicative of the associated theory uncertainty. As will become obvious the situation is complex and each experiment faces very specific challenges, and while the challenges are specific the solutions likely are not.

\subsection{The impact on the mixing angle measurement at $T 2 \mathrm{~K}$}

Many of the techniques discussed in this subsection have been developed for applications to the so-called $\beta$-beams [156], and can also be applied to real data, with the intent to estimate the systematic effects introduced in the analysis from the non perfect knowledge of the neutrino-nucleus cross section [157]. A tentative step along this line has been undertaken in the work of Ref. [158], where a three-flavor fit to the recent $v_{\mu} \rightarrow v_{e}$ and $v_{\mu} \rightarrow v_{\mu}$ T2K oscillation data with different models for the neutrino-nucleus cross section has been discussed. It was shown that, even with a limited statistics, the allowed regions and best fit points in the $\left(\theta_{13}, \delta_{C P}\right)$ and $\left(\theta_{23}, \Delta m_{\text {atm }}^{2}\right)$ planes are affected if, instead of using the RFGM to describe the QE cross section, a model including multi-nucleon emission processes was employed.

The sample of analyzed data comprises the $v_{\mu} \rightarrow v_{e}$ appearance [9] and $v_{\mu} \rightarrow v_{\mu}$ disappearance [5] modes; in the first case, 28 events passed all the selection criteria, implying (for a normal ordering case):

$$
\sin ^{2}\left(2 \theta_{13}\right)_{T 2 K}=0.14,
$$

with the $\mathrm{CP}$ phase $\delta_{C P}$ undetermined. In the disappearance channel, the 120 events collected by T2K were fitted with:

$$
\begin{aligned}
\left(\sin ^{2} \theta_{23}\right)_{T 2 K} & =0.51, \\
\left|\Delta m_{\text {atm }}^{2}\right|_{T 2 K} & =2.51 \cdot 10^{-3} \mathrm{eV}^{2} .
\end{aligned}
$$

In this case different models were considered, involving not only QE interactions but also pion production and inclusive cross sections. On the one hand, it was chosen a model as similar as possible to the one used by the T2K Collaboration. The T2K Collaboration simulates neutrino-nucleus interactions using the NEUT Monte Carlo Generator [159]. Even if the details of NEUT - as well as the effects of the latest tunings performed by the $\mathrm{T} 2 \mathrm{~K}$ Collaboration to take into account the recent measurements of K2K [122, 160], MiniBooNE [12, 161] and SciBooNE [162,163]—are not known, exclusive channels were treated using the same models implemented in NEUT. As a consequence, the RFGM [164] was used for the QE channel and the Rein and Sehgal model [165] was used for pion production. The second model considered in the analysis was the one developed by the authors of Ref. [166], that in the following will be referred to as RPA-2p2h, (see Section 3).

In the following, cross sections obtained using the two different approaches described above will be used. Those cross 
sections were computed in several exclusive channels (quasielastic and pion production), as well as in the inclusive one, for both charged current (CC) and neutral current (NC) interactions on carbon and oxygen (the targets used in near and far T2K detectors, respectively) and for two different neutrino flavors $v_{\mu}$ and $v_{e}$. Although all exclusive channels are involved in the analysis, the first model will be referred to as RFGM and the second approach as RPA-2p2h model.

In order to perform the comparisons among the abovementioned models, the RFGM was firstly normalized to the T2K event rates, at both near (ND) and far (FD) detectors using the following algorithm:

1. Normalization of the cross section with the $v_{\mu}$ inclusive $\mathrm{CC}$ at the ND; according to Ref. [5], in order to reproduce $\sim 1.8 \times 10^{4} v_{\mu}$ inclusive events, collected using $\sim 6.4 \times 10^{20}$ POT, in the energy range $[0-3] \mathrm{GeV}$, with an active detector mass of $1,529 \mathrm{~kg}$ at a distance of $280 \mathrm{~m}$ from the $v$ source and half a year of data taking (Run 1). Since only the muon neutrino cross sections can be correctly normalized, it was assumed that the same normalization also applies for the $v_{e}$ cross section, although they could differ at the $\mu$ production threshold (in any case away from the peak of the neutrino flux);

2. Calculation of the expected events (and energy distributions) at the far detector in the appropriate two-parameter plane $\left(\left(\sin ^{2} 2 \theta_{13}, \delta_{C P}\right)\right.$ for appearance and $\left(\theta_{23}, \Delta m_{a t m}^{2}\right)$ for disappearance);

3. Normalization to the $\mathrm{T} 2 \mathrm{~K}$ spectral distributions.

Step 3 is needed to get rid of the experimental efficiencies to the signal and background events. This means that the bin contents of the simulated distributions (obtained at step 2) are corrected by coefficients, generally of $O(1)$, that were considered as a detector property, and then not further modified. For a different model, step 1 was first redone, and then step 2 was repeated, using the same normalization coefficients extracted in step 3 with the RFGM. GLoBES [167, 168] and MonteCUBES [169] were the softwares used for the computation of event rates (and related $\chi^{2}$ functions) expected at the T2K ND and FD. The fluxes of $v_{\mu}, v_{e}$ and their CP-conjugate counterparts predicted at the FD in absence of oscillations were extracted directly from Fig. 1 of Ref. [170], whereas the $v_{\mu}$ flux at the ND was obtained from the authors of Ref. [171].

The appearance channel. The $v_{\mu} \rightarrow v_{e}$ transition probability is particularly suited for extracting information on $\theta_{13}$ and $\delta_{C P}$; at the T2K energies $\left(E_{v}\right)$ and baseline $(\mathrm{L})$, the full 3-flavor probability can be expanded up to second order in the small parameters $\theta_{13}, \Delta_{12} / \Delta_{13}$ and $\Delta_{12} L$, with $\Delta_{i j}=\Delta m_{i j}^{2} / 4 E_{v}$ [172]. The resulting expression is

$$
\begin{aligned}
& P_{v_{\mu}} \rightarrow v_{e}=s_{23}^{2} \sin ^{2} 2 \theta_{13} \sin ^{2}\left(\Delta_{a t m} L\right) \\
& \quad+c_{23}^{2} \sin ^{2} 2 \theta_{12} \sin ^{2}\left(\Delta_{\text {sol }} L\right) \\
& \quad+\tilde{J} \cos \left(\delta_{C P}+\Delta_{\text {atm }} L\right)\left(\Delta_{\text {sol }} L\right) \sin \left(2 \Delta_{\text {atm }} L\right)
\end{aligned}
$$

where

$$
\tilde{J} \equiv c_{13} \sin 2 \theta_{12} \sin 2 \theta_{23} \sin 2 \theta_{13}, s_{23}=\sin \theta_{23} .
$$

We clearly see that $\mathrm{CP}$ violating effects are encoded in the interference term proportional to the product of the solar mass splitting and the baseline, implying a weak dependence of this facility on $\delta_{C P}$ when only the $v_{\mu} \rightarrow v_{e}$ channel (and the current luminosity) is considered.

Extracting the T2K data. Events in the far detector are $v_{e} \mathrm{CC}$ $\mathrm{QE}$ from $v_{\mu} \rightarrow v_{e}$ oscillation, with the main backgrounds given by $v_{e}$ contamination in the beam and neutral current events with a misidentified $\pi^{0}$. The experimental data have been grouped in 25 reconstructed-energy bins, from 0 to $1.25 \mathrm{GeV}$. The expectations for signal and backgrounds have been computed by the T2K Collaboration from Monte Carlo simulations, for the following fixed values of the oscillation parameters:

$$
\sin ^{2} \theta_{12}=0.306, \sin ^{2} 2 \theta_{13}=0.14, \sin ^{2} 2 \theta_{23}=1,
$$

and

$$
\Delta m_{\text {sol }}^{2}=7.6 \times 10^{-5} \mathrm{eV}^{2}, \Delta m_{\text {atm }}^{2}=+2.4 \times 10^{-3} \mathrm{eV}^{2} .
$$

In order to normalize the event rates to the T2K Monte Carlo expectations, few numerical value were extracted from Table I of Ref. [9]. For the sake of simplicity, the central value was used as the reference value for the neutrino energy in a given bin; this could be different from the reconstructed neutrino energies used by the T2K Collaboration. To mimic possible uncertainties associated with the neutrino energy reconstruction, an energy smearing function was used to distribute the event rates in the various energy bins. The ratios among the computation presented in Ref. [158] and the T2K data define a sort of energy dependent efficiencies, $\varepsilon$, which, for the $v_{\mu} \rightarrow v_{e}$ signal turn out to be $\varepsilon \sim 0.4$. This procedure (corresponding to step 3 of the previous paragraph) allows to take into account all the detection efficiencies to different neutrino flavors in the Super Kamiokande detector. Once computed, these corrective factors are used in the simulations done with a different cross section model, since it was assumed that those are due to detector features and not to the neutrino interactions.

Fit to the data. Using these results, the authors of Ref. [158] performed a $\chi^{2}$ analysis to reproduce the allowed regions of the $\left(\sin ^{2} 2 \theta_{13}, \delta_{C P}\right)$-plane as shown in Fig. 5 of Ref. [9], based on a complete three-neutrino analysis of the experimental data shown in Fig. 4 of the same paper, marginalising over all parameters not shown in the confidence regions. As external input errors, the following list of parameters was used: $3 \%$ on $\theta_{12}$ and $\Delta m_{\text {sol }}^{2}, 8 \%$ on $\theta_{23}$ and $6 \%$ on $\Delta m_{\text {atm }}^{2}$. In addition, a constant energy resolution function $\sigma\left(E_{v}\right)=0.085$ was used and, for simplicity, a $7 \%$ normalization error for the signal and $30 \%$ for the backgrounds were adopted. The energy calibration errors were fixed to $10^{-4}$ for the signal and $5 \cdot 10^{-2}$ for the backgrounds; normalization and energy calibration errors were taken into account in evaluating the impact of systematic errors in the $\chi^{2}$ computation. 
Assuming a normal hierarchy spectrum, the best-fit point from the fit procedure is:

$$
\sin ^{2}\left(2 \theta_{13}\right)=0.126, \delta_{C P}=0.45
$$

with $\chi_{\min }^{2}=19.8$. Compared to the official release, the best fit points are in quite good agreement.

The same procedure was then applied to determine $\theta_{13}$ using the RPA-2p2h cross sections described in Ref. [166]. In doing that, the cross sections were normalized to the ND events and then the number of oscillated events (and related backgrounds) were computed, and compared with the experimental $\mathrm{T} 2 \mathrm{~K}$ data. Assuming that the energy dependent efficiencies computed in the previous section are exactly the same, since they are a property of the SK detectors, and therefore independent of the cross section model, and considering that the CC RPA-2p $2 \mathrm{~h}$ cross section is a bit larger than the RFGM cross section, a larger bin-to-bin rate was obtained, for a total of 33 events (signal plus backgrounds).

It is clear that larger rates need smaller $\theta_{13}$ to reproduce the data (the effect of the CP phase $\delta$ is negligible with such a statistics). The best fit point is:

$$
\sin ^{2}\left(2 \theta_{13}\right)=0.08 \quad, \quad \delta_{C P}=0,
$$

with $\chi_{\text {min }}^{2}=19.2$. To make a more direct comparison on $\theta_{13}$ between RFGM and RPA-2p2h results, in Fig. 34 we show the function $\chi^{2}-\chi_{\text {min }}^{2}$, computed marginalizing over all other oscillation parameters (including $\delta_{C P}$ ). At $1 \sigma$ level, the following result was obtained:

$$
\sin ^{2} 2 \theta_{13}^{R P A-2 p 2 h}=0.11_{-0.06}^{+0.03}, \sin ^{2} 2 \theta_{13}^{F G}=0.14_{-0.06}^{+0.05} .
$$

The results are clearly compatible although, as expected, $\theta_{13}^{R P A-2 p 2 h}<\theta_{13}^{F G}$.

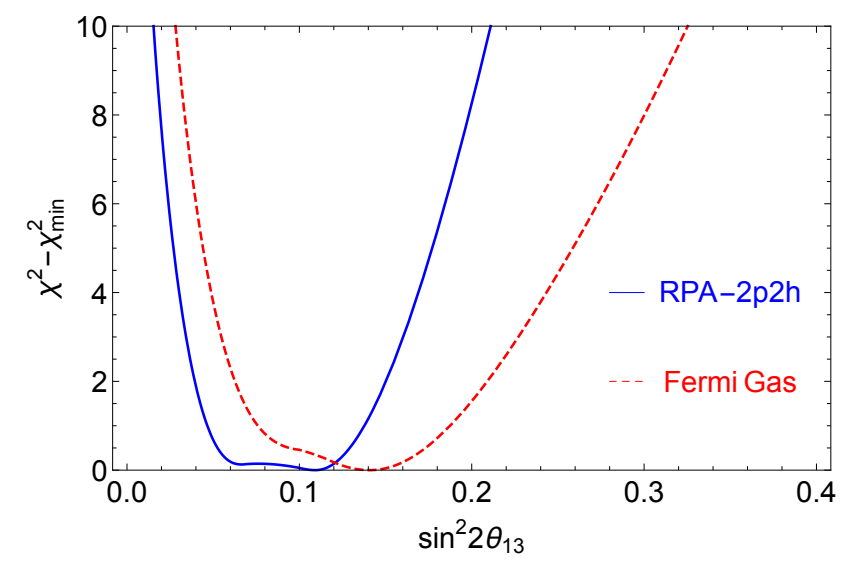

Figure 34: Behavior of the function $\chi^{2}-\chi_{\min }^{2}$ (see text) as a function of $\theta_{13}$, for the RPA-2p2h model (solid line) and the RFGM (dashed line).

The disappearance channel. The previous analysis was then extended to include the disappearance $v_{\mu} \rightarrow v_{\mu}$ data [5]. In the two-flavor limit, (the one where both $\theta_{13}$ and $\Delta m_{\text {sol }}^{2}$ are vanishing) the $v_{\mu} \rightarrow v_{\mu}$ probability reads [173]:

$$
P\left(v_{\mu} \rightarrow v_{\mu}\right)=1-\sin ^{2} 2 \theta_{23} \sin ^{2}\left(\Delta_{a t m} L\right) .
$$

Effects related to $\theta_{13}$ are clearly sub-dominant, so that this channel is particularly useful to extract information on the atmospheric parameters. The T2K Collaboration collected 120 data events, grouped in 30 energy bins, as one can see from Fig. 2 of [5]. The sample extends above $5 \mathrm{GeV}$ and is mainly given by $v_{\mu} \mathrm{CC} \mathrm{QE}, v_{\mu} \mathrm{CC}$ non-QE, $v_{e} \mathrm{CC}$ and NC. The RFGM cross section was normalized to the rates shown in Fig. 2 of [5]. In the fit procedure a conservative $15 \%$ normalization error and an energy calibration error at the level of $10^{-3}$ for both the signal and the background were adopted. The results of the analysis are shown in Fig. 35, where it can be found the 90\% CL limit for the RFGM (dashed line) and the RPA-2p2h model (solid line), in the case of normal hierarchy together with the 2 degrees of freedom (dof) confidence levels in the $\left(\theta_{23}, \Delta m_{a t m}^{2}\right)$-plane. Again, the plots have been obtained marginalizing over the parameters that are not shown (a full three-flavor analysis). The plot in Fig. 35 was obtained considering a $50 \%$ error on $\sin ^{2} 2 \theta_{13}$ (with best fit at $\sin ^{2} 2 \theta_{13}=0.14$ ) and $\delta_{C P}$ undetermined.

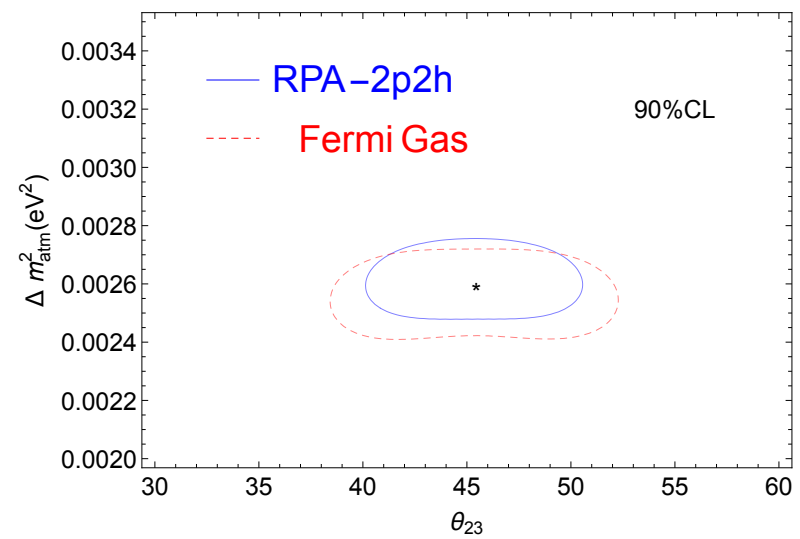

Figure 35: $90 \%$ contour levels for the RPA-2p2h model (solid line) and the RFFM (dashed line), in the $\left(\theta_{23}, \Delta m_{\text {atm }}^{2}\right)$ plane. Star indicates the best fit obtained in the RPA-2p2h model.

In summary the following results were obtained:

$$
\begin{aligned}
& R F G: \\
& 38<\theta_{23}<52 \mathrm{deg}, \\
& 2.40 \cdot 10^{-3}<\Delta m_{\text {atm }}^{2}\left(\mathrm{eV}^{2}\right)<2.70 \cdot 10^{-3}, \\
& R P A-2 p 2 h: \\
& 40<\theta_{23}<50 \mathrm{deg}, \\
& 2.50 \cdot 10^{-3}<\Delta m_{\text {atm }}^{2}\left(\mathrm{eV}^{2}\right)<2.80 \cdot 10^{-3},
\end{aligned}
$$

with best fit points:

$R F G$ :

$\theta_{23}=47.9 \mathrm{deg}, \Delta m_{\text {atm }}^{2}=2.56 \cdot 10^{-3} \mathrm{eV}^{2}$

$R P A-2 p 2 h:$

$\theta_{23}=45.4 \mathrm{deg}, \Delta m_{\text {atm }}^{2}=2.62 \cdot 10^{-3} \mathrm{eV}^{2}$. 
Here, some comments are in order. First of all, we should observe that, for both models, the best fit point is different from the $\mathrm{T} 2 \mathrm{~K}$ one, which corresponds to

$$
\left(\theta_{23}\right)_{T 2 K}=45.8 \mathrm{deg},\left|\Delta m_{a t m}^{2}\right|_{T 2 K}=2.51 \cdot 10^{-3} \mathrm{eV}^{2} .
$$

This is somehow obvious since we normalized our events to the MC predictions obtained for a different set of atmospheric parameters. The RPA-2p $2 \mathrm{~h}$ cross section gives a better determination of both $\theta_{23}$ and $\Delta m_{\text {atm }}^{2}$, mainly due to the larger statistics with respect to the RFGM; at the same time, the disappearance probability in Eq.(79), for negligible solar mass difference and reactor angle, is smaller if the atmospheric mass difference is larger, for fixed $\sin ^{2} 2 \theta_{23}$. This is what happens here, where a smaller $P\left(v_{\mu} \rightarrow v_{\mu}\right)$ (and then a larger $\left.\Delta m_{\text {atm }}^{2}\right)$ is needed in the RPA-2p $2 \mathrm{~h}$ model to partially compensate for the larger cross section.

For the sake of completeness, the same computations were repeated as above under the hypothesis that the neutrino mass spectrum is of inverted type (IH). With the current T2K statistics, one does not find significant differences in the results obtained using the two different models for the cross section.

\subsection{Reconstruction of neutrino energy}

Future long-baseline neutrino experiments will rely on their ability to map the energy dependence of the oscillation probability to measure oscillation mixing parameters and test the validity of the three-flavor oscillation framework.

The oscillation probability is a non-trivial function of the true neutrino energy, and thus the problem of reconstructing the neutrino energy arises. Also, the fact that some experiments seem to be less affected by rate-only systematics is largely due to their ability to exploit the differences in energy dependence of the various contributions to the error budget to control systematic uncertainties. There are a number of works [175-178] where it is shown that event rate distributions in reconstructed neutrino energy will change significantly based on the underlying interaction model. A conceptual laboratory is provided by quasi-elastic scattering, which, due its relative simplicity and amenability to theoretical calculations, has been also the focus of many published studies. In a true quasi-elastic scattering event involving a neutron at rest there is a one-to-one correspondence between the charged lepton momentum and emission angle and the incoming true neutrino energy, given by

$$
E_{v}=\frac{2\left(m_{n}-\epsilon\right) E_{\ell}-\left(\epsilon^{2}-2 m_{n} \epsilon+m_{\ell}^{2}+\Delta m^{2}\right)}{2\left(m_{n}-\epsilon-E_{\ell}+\left|\mathbf{k}_{\ell}\right| \cos \theta_{\ell}\right)} .
$$

In the above equation, $\mathbf{k}_{\ell}$ and $E_{\ell}$ are the momentum and energy of the outgoing charged lepton, $\theta_{\ell}$ is the scattering angle in the laboratory frame, $\Delta m^{2}=m_{n}^{2}-m_{p}^{2}$ is the neutron-proton squared mass difference and $\epsilon$ denotes the average binding energy of the neutron.

The problem, now, is that in any experiment there will be events which are not quasi-elastic but exhibit all the same experimental signatures of a true quasi-elastic event, e.g. the socalled stuck-pion events where in addition to the charged lepton a pion is produced at the vertex, but this pion is then re-absorbed within the nucleus. As a result, any real QE event sample will contain non-QE events as well, and for those non-QE events the simple kinematic relation in Eq. (80) will not be valid.

In a water Cherenkov detector the selection criterion for $\mathrm{QE}$ events is that only one charged particle is above Cherenkov threshold, resulting in a single ring of light. Taking the output of an event generator and selecting events using this criterion it is possible to construct the appropriate migration matrix between true and reconstructed energy, which, if the generator were perfect, would completely describe these effects. In practice, different generators lead to very different migration matrices and, as a result, to very different reconstructed energy distributions in both near and far detectors, as shown in Fig. 36. Interestingly, an offset in the distributions between GENIE and GiBUU is observed and the overall effect is to change the position and depth of the oscillation dip. Similar effects have been observed previously by several authors [175-178]. The next question to address is what will the impact on the extraction of oscillation parameters be, and whether the inclusion of the near detector will solve the problem.

A first step in this direction was taken by the authors of Ref. [179], where a comparison between ideal energy reconstruction and a fit performed using migration matrices derived from an event generator-in this case GiBUU-was made. Specifically, the ability to measure the atmospheric mixing parameters in $v_{\mu} \rightarrow v_{\mu}$ disappearance was studied. The main finding is that the use of a near detector leads to a high $\chi^{2}$-value per degree of freedom, that is a bad fit, but does not prevent significant bias in the parameter determination, which can potentially be as large as several times the statistical error. Both the $\chi^{2}$-value and bias can be reduced if the energy scale of the experiment is allowed to shift by as much as 5\%. The key to this behavior is the fact, that the beam flux is not known a priori to better than $5-10 \%$ and hence the near detector cannot determine the right energy migration matrix and the beam flux simultaneously. In essence, there are fewer observables than unknowns.

A somewhat more sophisticated analysis has been presented in Ref. [174], where a comparison between two event generators, GiBUU and GENIE, was performed. One of the generators was used to compute virtual data, and this virtual data was in turn fitted using the other event generator. The specific choice of generators for this comparison was guided by convenience and availability and does not imply that one of them is more accurate than the other, or that any of them is more accurate than some other generator. The results are somewhat sobering, as can be seen from Fig. 37, clearly indicating that a large bias with acceptable $\chi^{2}$-values could occur. The closed contours are obtained from fitting data generated with GENIE with GENIE, whereas the open contours are obtained from fitting data generated with GENIE with GiBUU. In the left hand panel the energy scale is fixed, while in the right panel a $5 \%$ energy scale shift is allowed.

The authors of Ref. [178] pointed out that an event sample which is the combination of 0-pion events (traditionally selected as QE), 1-pion and N-neutron events has a much more 

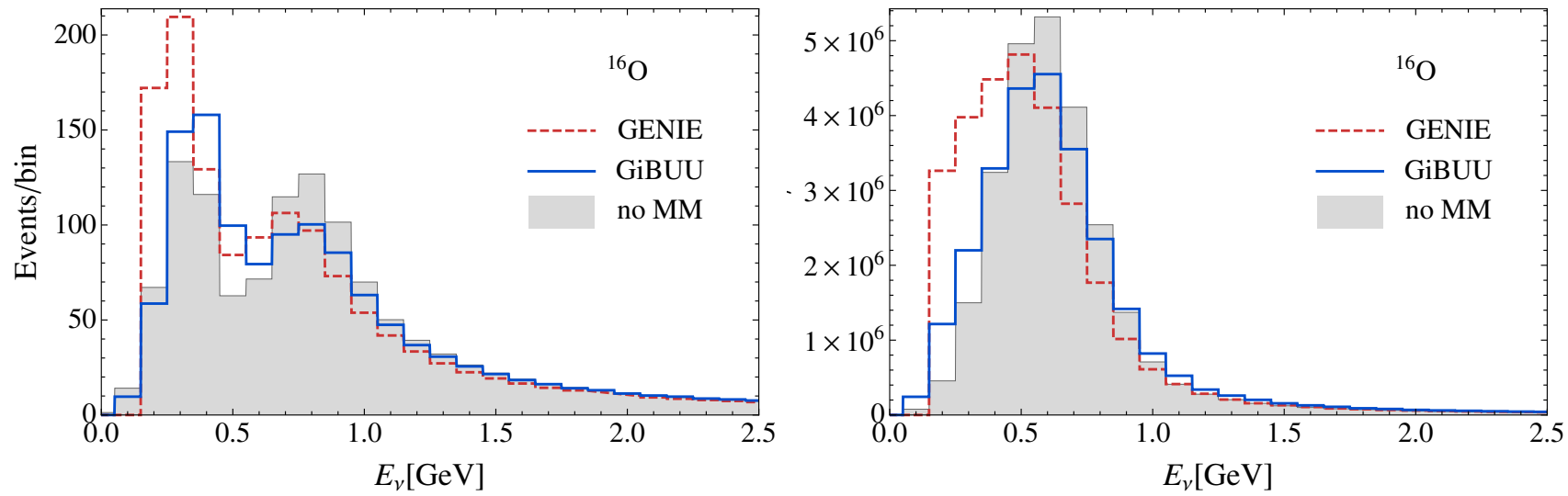

Figure 36: Binned QE-like event rates as a function of the reconstructed neutrino energy in GeV. The solid (dashed) line shows the event rates obtained after migration using the GiBUU (GENIE) event generator. The shaded areas show the expected event rates coming from the QE-like event sample computed using the GiBUU cross-section for ${ }^{16} \mathrm{O}$, as for the solid lines, but without including any migration matrices. For the shaded areas, a Gaussian energy resolution function with a constant standard deviation of $85 \mathrm{MeV}$ is added to account for the finite resolution of the detector. The left and right panels show the event rates at the near and far detectors, respectively. Figure and caption adapted from Ref. [174].
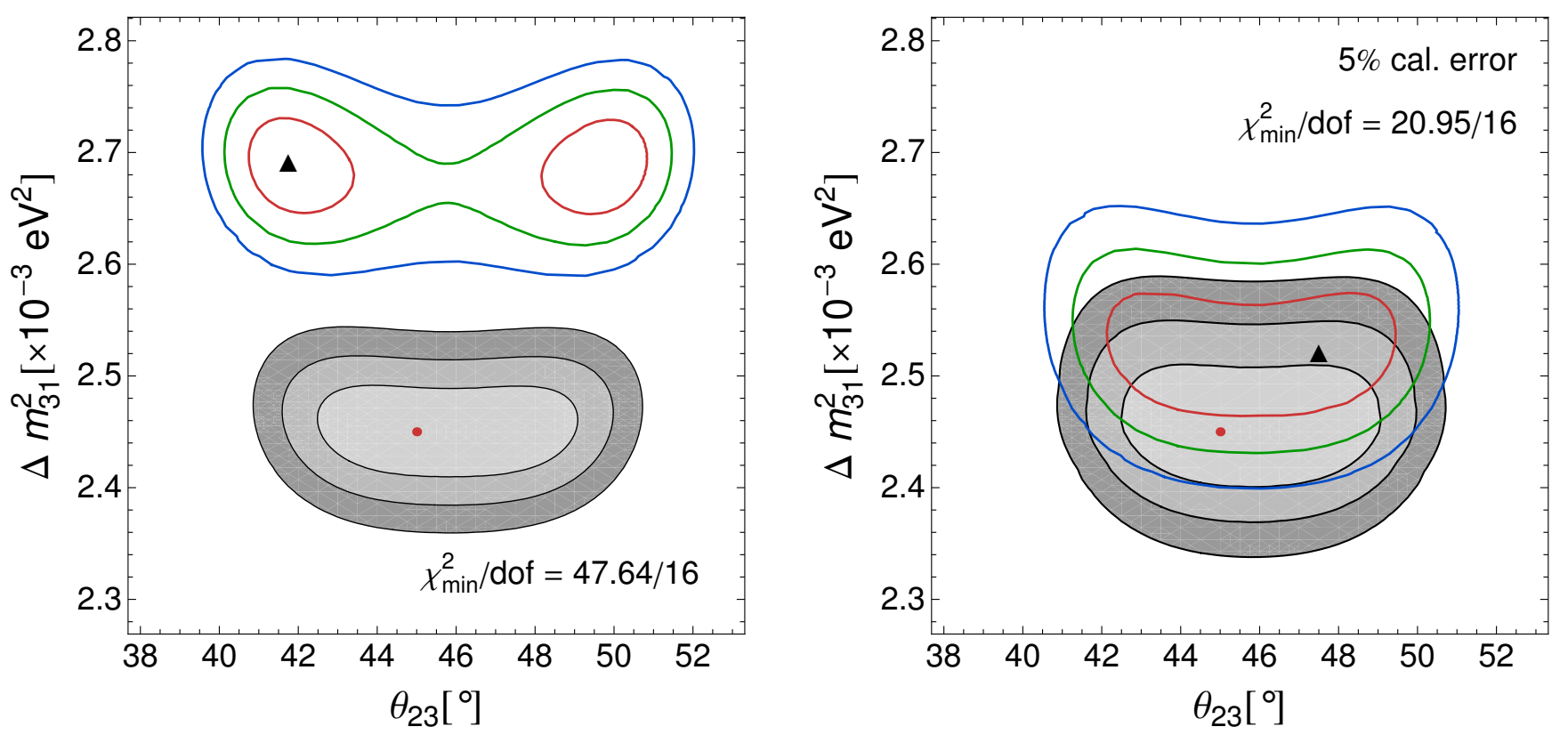

Figure 37: Impact on the results if a different generator is used to compute the true and fitted rates in the analysis. The shaded areas show the confidence regions at 1,2 and $3 \sigma$ that are obtained in the $\theta_{23}-\Delta m_{31}^{2}$ plane if the true and fitted rates are generated using the same set of migration matrices (obtained from GiBUU, with oxygen as the target nucleus). The solid lines show the same confidence regions if the true rates are generated using matrices produced with GiBUU, but the fitted rates are computed using matrices produced with GENIE. Both sets of matrices are generated using oxygen as the target nucleus. The dot indicates the true input value, while the triangle shows the location of the best fit point. The value of the $\chi^{2}$ at the best fit is also shown, together with the number of degrees of freedom. In the left panel no energy scale uncertainty is considered, while for the right panel an energy scale uncertainty of $5 \%$ is assumed, see text for details. Figure and caption adapted from Ref. [174] 
benign behavior in terms of shifting the oscillation peak. It is clear, that such a selection would require a liquid argon detector. Moreover, the statistics is strongly reduced with respect to the full event sample in an experiment in the multi-GeV region. There is some risk that an improved systematic error is bought at the price of a greatly enlarged statistical error. On the other hand, having a reliable sub-sample may be sufficient to also tie down the energy scale of DIS events. This issue awaits further detailed study along the lines of Ref. [174].

The results of Ref. [178] indicate that a more detailed treatment of the hadronic energy deposition, which can be measured to a large extent in liquid argon detectors, will likely improve the results. At the same time, missing energy will become a crucially important problem. Neutral secondary particles like $\pi^{0}$ and neutrons have to either decay or interact further in the detector to leave a signature. The amount of these neutral secondaries will be very different in neutrino and antineutrino interactions, as will their energy distributions. This can be easily seen from the very different $y$-distributions in DIS for neutrinos and antineutrinos. The contained fraction of neutral particles will be a sensitive function of detector size and surface to volume ratio. Therefore near and far detectors are guaranteed to behave very differently in this respect. Even if neutral particles are contained in the detector, associating their signature with the (correct) primary vertex will be complicated, in particular in the near detector, which may see more than one event per beam spill.

A further real-world issue will be energy thresholds, implying that a proton has to exceed a certain value of kinetic energy in order to be detected. The relatively poor energy resolution for hadronic energy deposition, compared to the one for leptons, will impose further limitations. A quantitative study of the impact of hadronic calorimetry with a special emphasis on the missing energy from neutral secondaries is urgently needed. This also implies the need for reliable theoretical models-most notably of neutral particle production in neutrinonucleus interactions-capable to correctly predict multiplicity and momentum distributions, since they affect the fraction of contained events and their signature. This information is required to correctly account for the aforementioned thresholds and the hadronic energy resolution, even if detailed test beam data on the detector response exist. Furthermore, it is obvious that the energy scales and systematic bias for electronand muon-type events will be quite different, which adds another level of complexity, an will cause practical difficulties for attempts to use the disappearance data, typically based on $v_{\mu} \rightarrow v_{\mu}$, to "calibrate" the energy scale of the appearance data set, based on $v_{\mu} \rightarrow v_{e}$. It is also worth noting that this approach effectively assumes the correctness of the three flavor oscillation framework, thus defeating one the major reasons for pursuing long-baseline experiments: tesing the validity of the three-flavor description.

\subsection{Detector effects impact on disappearance and appearance results}

As we have seen in the previous sections in long/short baseline neutrino oscillation experiments neutrino energy is the key element. The neutrino energy is reconstructed looking at the kinematics of particles produced in the neutrino interaction with the detector. There are two reconstruction techniques: one, already described in Eq. (80), is based on the QE assumption and only uses the information on muon momentum. We will call this method kinematical. A different technique to reconstruct the energy, that we will call calorimetric, consists in collecting all the energy of the particles deposited in the detector, and from that infer the neutrino energy. In both methods the reconstructed neutrino energy will depend upon detection capabilities, such as energy resolutions, reconstruction efficiencies and detector energy thresholds for the different particles.

The impact of detector capabilities on the oscillation analysisIn has been recently investigated in Ref. [180, 181]. The authors studied how uncertainties related to the detector effects influence the oscillation analysis by using event distributions simulated in the far detector which include detector effects and analyzing them neglecting, or partially neglecting, detector effects in the final oscillation analysis.

In Ref. [180, 181] the kinematical observables have been smeared according to a normal distributions centered at their true values. For muons, the smearing was applied to both the momentum and emission angle, using the following realistic parameters [182]:

$$
\sigma\left(\left|\mathbf{k}_{\mu}\right|\right)=0.02\left|\mathbf{k}_{\mu}\right| \quad \text { and } \quad \sigma(\theta)=0.7^{\circ} .
$$

The energy resolutions of for $\pi^{0}$ 's producing electromagnetic showers and other hadrons have been set to

$$
\begin{aligned}
\frac{\sigma\left(E_{\pi^{0}}\right)}{E_{\pi^{0}}} & =\max \left\{\frac{0.107}{\sqrt{E_{\pi^{0}}}}, \frac{0.02}{E_{\pi^{0}}}\right\}, \\
\frac{\sigma\left(E_{h}\right)}{E_{h}} & =\max \left\{\frac{0.145}{\sqrt{E_{h}}}, 0.067\right\},
\end{aligned}
$$

respectively, with detection thresholds corresponding to a measured kinetic energy of $20 \mathrm{MeV}$ for mesons and $40 \mathrm{MeV}$ for protons. The efficiencies, on the other hand, have bee considered as energy independent and set to $60 \%$ for $\pi^{0}$ 's, $80 \%$ for other mesons, and $50 \%$ for protons. Neutrons were assumed to always escape detections.

In the context of the $v_{\mu}$ disappearance analysis [180], the authors analyzed the T2K-like setup described in Section 7.2, with a number of expected unoscillated events of $\sim 4900$.

Instead of changing individual parameters related to the detector performances, the authors used a linear combination of migration matrices computed with and without detector effects.

As shown in Fig. 38, the kinematic method turns out to be mostly independent of detector effects, and uncertainties at the level of $30 \%$ (corresponding to $\alpha=0.3$ ), do not significantly affect the results of the oscillation analysis, due to the good resolution in the determination of the muon kinematics.

In the case of calorimetric energy reconstruction, on the other hand, the authors of Ref. [180] see a large dependence of the neutrino energy on detector effects. Figure 39 shows that the detector response has to be determined with high precision-at least 10\% - to avoid large biases in the determination of the oscillation parameters. This behavior is mainly due to the 

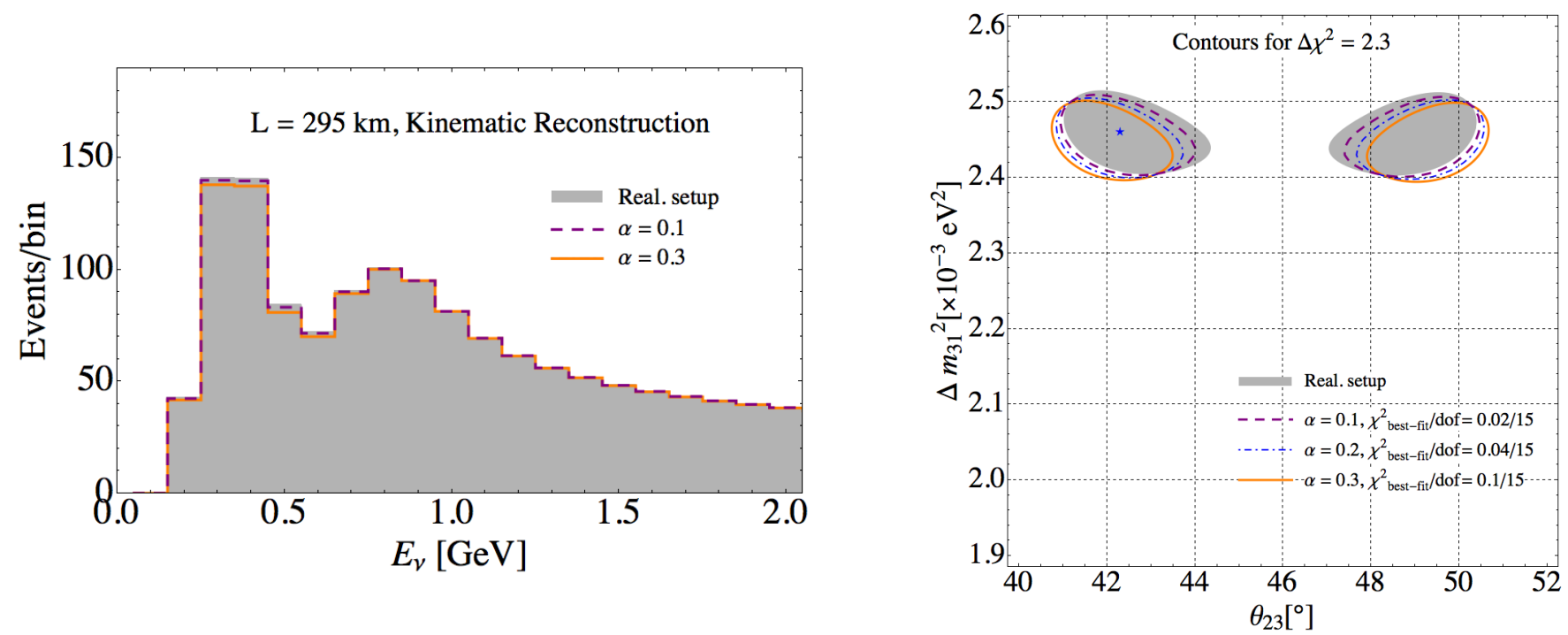

Figure 38: Effect of detector-related uncertainties on the oscillation analysis performed using kinematic energy reconstruction. Left: comparison between the event distributions obtained overestimating the detector performance by $10 \%$ ( $\alpha=0.1$, dashed line) and by 30\% ( $\alpha=0.3$, solid line). The accurate estimate of detector effects is represented in the shaded histogram. Right: $1 \sigma$ confidence regions in the $\left(\theta_{23}, \Delta m_{31}^{2}\right)$ plane obtained using data simulated including detector effects and oscillation parameters extracted using migration matrices with 100\% detector effects (shaded area) and at 10\%, 20\%, 30\% level only (lines).
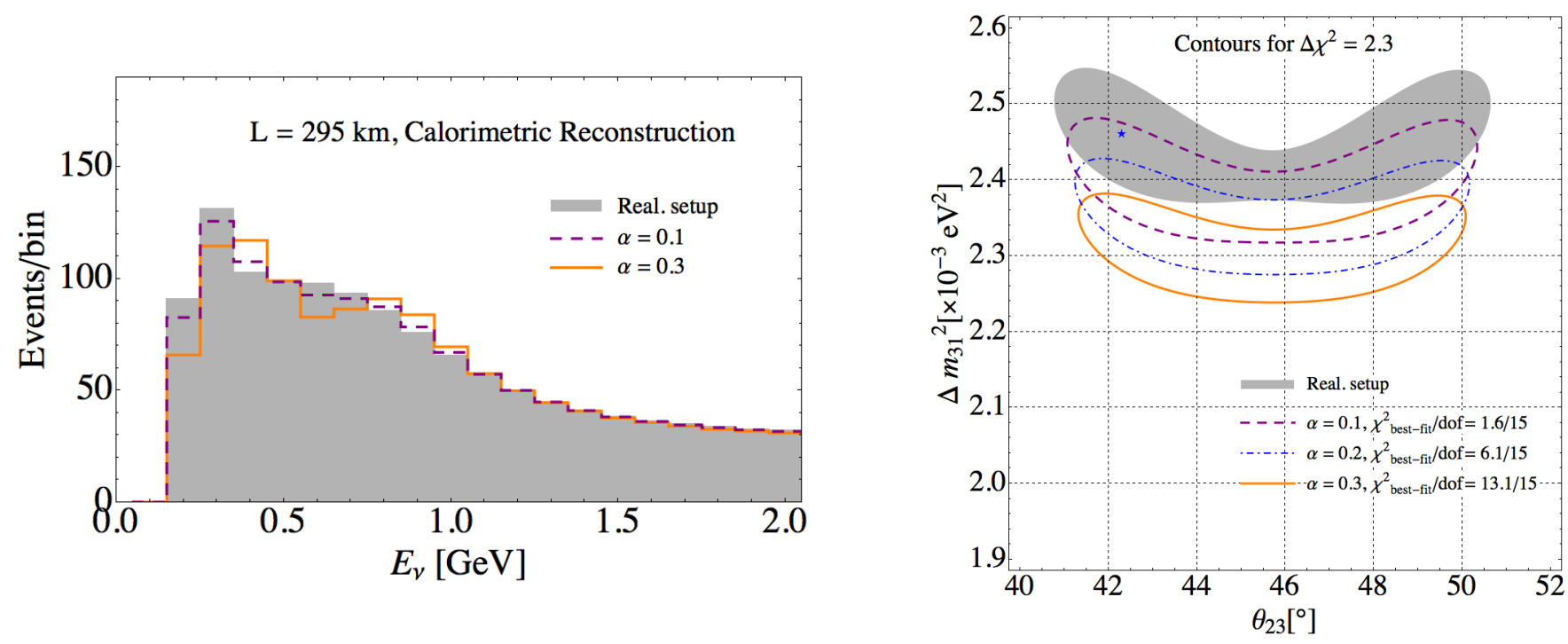

Figure 39: Same as figure 38 but considering the calorimetric energy reconstruction. 
large uncertainties in the determination of hadron energies, see Eq. (82), associated with the calorimetric technique.

Although the results of Figs. 38 and 39 have been obtained for beam peaked at $\sim 0.6 \mathrm{GeV}$ [183], the above conclusions can be extended to the case of a wide-band beam peaked at $\sim 1.6$ $\mathrm{GeV}$, as discussed in Ref. [180].

The same effects have been studied in the case of oscillation experiments aimed at accurately measuring $\delta_{C P}$. In this instance the oscillation probability is more complicated, due to the presence of different neutrino/anti-neutrino species (like electron (anti)neutrinos from oscillations of muon (anti)neutrinos). The authors of Ref. [181] analyzed the role of missing energy on the $\delta_{C P}$ sensitivity for an experiment similar to DUNE [184].

In the DUNE-like setup, a wide-band neutrino beam has been analyzed with a far detector of 40 kton (fiducial mass), located $1300 \mathrm{~km}$ from the neutrino beam. The authors assumed 6 years of running, 3 years in neutrino mode and 3 years in antineutrino mode. For the signal, $2 \%$ uncertainties of normalization (bin-to-bin correlated) and shape (bin-to-bin uncorrelated) were considered, and in the case of the background only a global normalization uncertainty of 5\% was taken into account [181].

The event distributions have been simulated including all detector effects-resolutions, efficiencies, and energy thresholds. The oscillation parameters were extracted using linear combinations of migration matrices calculated with and without missing energy shifts.

From the left panel of Fig. 40 it is evident that the maximum of the distribution in energy is shifted to values lower than the true neutrino energy, due to the missing energy. As the nature of the particles in the final state is different for neutrinos and antineutrinos, this shift will be different, since it depends on the interaction channel, the momentum transfer and the nature of the neutral secondary particles which give rise to missing energy. As shown in the right panel of Fig. 40, a $20 \%$ underestimation of the missing energy introduces a large bias in the extracted $\delta_{C P}$ value. If, instead, the missing energy will be underestimated by $30 \%$, the oscillation analysis would exclude the true value of $\delta_{C P}$ at a confidence level between 2 and $3 \sigma$. This result illustrates the importance of an accurate determination of detector response in test-beam exposures and the relevance of a realistic simulation of nuclear effects in neutrino interactions, including intranuclear cascade.

In summary, a significant improvement of our theoretical understanding of neutrino-nucleus cross section is required, since the currently existing neutrino beams do no allow for measurements at a sufficient level of precision. This, in turn, raises the question of how to validate a theoretical model to a precision better than the available neutrino scattering data. One part of the answer, clearly can come from electron scattering data, since-irrespective of the underlying theoretical framework-any model able to predict the electro-weak nuclear response necessarily must also describe the electro-magnetic response, which can be accurately measured by electron scattering experiments. This is a necessary condition, but whether it is sufficient is difficult to judge. We are faced by a vicious cycle: the lack of high quality data necessitates theory to be trusted, but to trust theory it needs to be validated against data. It should be kept in mind that if everything else fails, vSTORM [145] provides a way out of this vicious cycle by "simply" providing very high quality data, for neutrino and antineutrino as well as for $v_{\mu}$ and $v_{e}$ interactions. This data, will likely be good enough to allow extrapolation to any experimental situation of interest. In the case extrapolation is insufficient, $v$ STORM data would provide the cornerstone to decisively test the theoretical understanding of neutrino-nucleus interactions.

At a phenomenological level, existing studies, of which a few examples were shown in this section, have merely started to scratch the surface of the issue and, at this stage, it is not clear, whether many results are not just a mere consequence of the assumptions put into the analysis. One example is, for instance, the relative robustness of LBNE-like experiments against cross section systematics found by the authirs of Ref. [155]: in light of later studies, this may be entirely due to using systematical uncertainties which only affect the rate but not the shape of the signal. The conceptually expedient simplification to focus on one interaction type, like QE, severely restricts practical applicability for future experiments in the multi-GeV energy range, where a multitude of interaction mechanisms contributes. Similarly, comparing different event generators may create a false sense of the magnitude of the problem, in particular since none of the existing generators is known to correctly describe neutrino scattering over a wide kinematic range and different interaction modes.

\section{Summary and outlook}

The surge of activities aimed at improving the description of neutrino-nucleus interactions, critical for the interpretation of oscillation signals, is now over a decade old, and still growing. Beginning with the first Workshop of the NUINT (NeutrinoNucleus Interactions in the Few GeV Region) series-that marked the dawning of the age of collaboration between the communities of nuclear theory and neutrino physics back in 2001-a number of experimental and theoretical developments contributed to steadily advance the field.

On the experimental side, the MiniBooNE Collaboration performed the first measurement of the double differential nuclear cross section in the QE sector [12], thus providing an unprecedented opportunity to test the available theoretical models and compare their predictions of the flux-integrated neutrino cross section. Additional information has been provided by the NOMAD [123, 185] and T2K [136, 186] Collaborations, as well as by the SciBooNE [187] and Minerva [15] experiments, specifically designed to study neutrino-nucleus interactions in different kinematical regions and using different nuclear targets.

Theoretical studies, carried out using highly advanced models of nuclear structure and dynamics, shed new light on the complex reaction mechanisms contributing to the flux integrated cross sections, the understanding of which is needed to reduce the uncertainties associated with event identification and neutrino energy reconstruction. In this context, processes involving two-nucleon correlations and meson-exchange currents appear to play a significant role. 

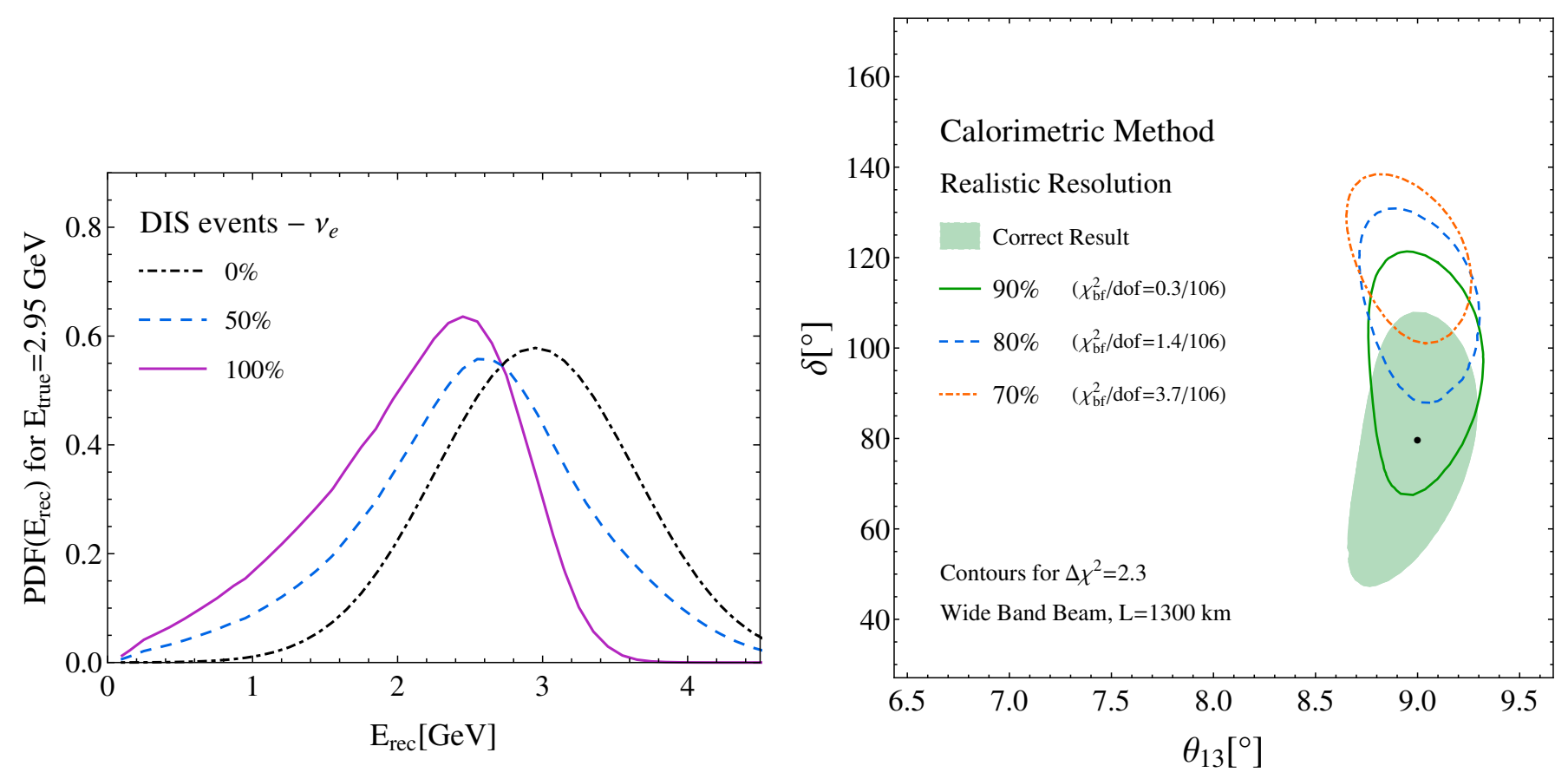

Figure 40: Impact of the missing energy on the oscillation analysis for a DUNE like setup using the calorimetric energy reconstruction. Left: Reconstructed energy distributions for DIS of electron neutrino at energy $2.95 \mathrm{GeV}$ (true), obtained including a $0 \%, 50 \%$ and $100 \%$ shift of the reconstructed energy due to the missing energy. Right: $1 \sigma$ confidence regions in $\left(\theta_{13}, \delta_{C P}\right)$ plane obtained using simulated data fitted using the migration matrices accounting for $90 \%, 80 \%$ and $70 \%$ (lines) of the missing energy and all the missing energy (shaded area). The dot shows the assumed true values of the oscillation parameters.

Thanks to the availability of ever more powerful computers, and to the steady evolution of Monte Carlo computational algorithms, accurate $a b$ initio calculations of scattering observables, based on the formalism of nuclear many-body theory and realistic nuclear Hamiltonians, can presently be carried out for nuclei as large as carbon. Pioneering calculations have been also carried out within the variational Monte Carlo approach using a relativistic Hamiltonian, although these studies are limited to the ground state energies of ${ }^{3} \mathrm{H}$ and ${ }^{4} \mathrm{He}$ [188]. For the foreseeable future, applications of the GFMC computational technique to the calculation of the nuclear response will be unavoidably limited to the region of low to moderate momentum transfer, in which the non relativistic approximation is expected to be workable. However, GFMC results will provide most valuable benchmarks to test the accuracy of more approximate approaches in the non relativistic limit.

Nuclear interactions at large momentum transfer are effectively described using the formalism based on the the factorisation ansatz. Within this scheme, the interaction vertex is treated using the full relativistic expression of the nuclear current, while the initial state-which is obviously independent of momentum transfer-is described in terms of non relativistic spectral functions. The development of improved models of the target spectral function, needed to study neutrino interactions in liquid argon detectors, will require both the experimental information coming from electron scattering experiments [16] and the use of Monte Carlo techniques to carry out accurate calculations of the relevant nuclear amplitudes.

The factorisation ansatz provides a fully consistent frame- work to treat processes involving both one- and two-nucleon currents. Moreover, to the extent to which the matrix elements of the current can be parametrized exploiting the available proton and deuteron data, it can be applied to all interaction channels: quasi elastic scattering, resonance production and deep inelastic scattering. This scheme is ideally suited for implementation in simulation codes, but disregards the effects of final state interactions between the particles produced at the interaction vertex and the spectator nucleons, which, depending on kinematics, may be quite significant. Extensive theoretical work on electron-nucleus scattering suggests that the effects of final state interactions can be systematically included within the spectral function formalism using the eikonal approximation. The existing applications of this approach, which allows for a consistent treatment of initial- and final-state correlations, are limited to the quasi elastic sector. However, the underlying conceptual scheme, based on the assumptions that (i) the struck nucleon travels along a straight trajectory with constant velocity, and (ii) the spectator nucleons can be seen as a collection of fixed scattering centers, has a much broader range of applications. Cascade Monte Carlo simulations, designed to provide an event-by-event description of the complex final states occurring in neutrino interactions at energies between few hundreds $\mathrm{MeV}$ and few $\mathrm{GeV}$, are in fact largely based on the same assumptions. The development of simulation codes whose basic inputs - coordinate-space distribution of the spectator nucleons and medium-modified hadronic cross sections-are consistent with the theoretical description of the target initial state based on many-body theory appears to feasible, and needs to be thor- 
oughly investigated.

The picture emerging from this review suggests that there are routes worth exploring to achieve a better quantitative understanding of what needs — and needs not—-to be known about neutrino-nucleus interactions for long-baseline oscillation experiments.

First, comparison between event generators will remain indicative of potential issues, and will help to pin down where there needs to be more theoretical work, or where existing theoretical results need to be implemented into generators. However, there is an exponential number of possibilities to combine various generators and their options. Therefore, care should be used to pick illuminating cases for comparison, instead of attempting to completely exhaust the possibilities (and likely the audiences as well). In this process, agreement between results from different generators should not be misinterpreted as a sign of correctness, since there is a distinct possibility of several generators being wrong about the same physics in the same or very similar way. For example, most generators rely on the RFGM as a description of the initial state.

It is worth mentioning that the above considerations also apply to the cross section models, which often predict very similar results in spite of being based on totally different, and sometimes incompatible, physics assumptions [189].

Second, completeness should come before accuracy. A complete cross section model which can cover all the relevant regions of kinematics and interaction modes at a coarse level of approximation is, at this stage, preferable to a microphysical accurate description of a narrow kinematic range or a single interaction channel. The devil is the in interaction of the various pieces, and many clever schemes to solve one particular problem eventually fail because of all the other moving parts. The same applies for the subsequent phenomenological analysis. For instance, flux uncertainties clearly limit what a near detector can do in terms of eliminating systematics [154].

Third, detector effects need to be included, even if only approximately. Thresholds, energy resolution and particle identification are all interrelated and typically rely on the underlying event generator.

Fourth, the three flavor oscillation should not be assumed since testing this framework is a major objective for the next generation of long-baseline experiments.

Obviously, achieving all the above goals in a combined analysis will require a strong synergy between experimentalists, theorists and developers of simulation codes.

\section{References}

[1] Q.R. Ahmad, et al. (SNO Collaboration), Phys. Rev. Lett. 89 (2002) 011301.

[2] T. Araki, et al. (KamLAND Collaboration), Phys. Rev. Lett. 94 (2005) 081801.

[3] Y. Ashie, et al. (Super-Kamiokande Collaboration), Phys. Rev. Lett. 93 (2004) 101801.

[4] E. Aliu, et al. (K2K Collaboration), Phys. Rev. Lett. 94 (2005) 081802.

[5] K. Abe, et al. (T2K Collaboration), Phys. Rev. Lett. 112 (2014) 181801.

[6] P. Adamson, et al. (MINOS Collaboration), Phys. Rev. Lett. 107 (2011) 181802 .
[7] F.P. An, et al. (Daya Bay Collaboration), Phys. Rev. Lett. 108 (2012) 171803.

[8] J.K. Ahn, et al. (RENO Collaboration), Phys. Rev. Lett. 108 (2012) 191802.

[9] K. Abe, et al. (T2K Collaboration), Phys. Rev. Lett. 112 (2014) 061802.

[10] R. Acciari, et al. (DUNE Collaboration), arXiv:1512.06148 [physics.ins-det] .

[11] K. Abe, et al. (T2K Collaboration), Phys. Rev. D 88 (2013) 032002.

[12] A.A. Aguilar-Arevalo, et al. (MiniBooNE Collaboration), Phys. Rev. D 81 (2010) 092005

[13] A.A. Aguilar-Arevalo, et al. (MiniBooNE Collaboration), Phys. Rev. Lett. 108 (2008) 191802.

[14] A.A. Aguilar-Arevalo, et al. (MiniBooNE Collaboration), Phys. Rev. D 88 (2013) 032001.

[15] B.G. Tice, et al. (MINER $v$ A Collaboration), Phys. Rev. Lett. 112 (2014) 231801.

[16] O. Benhar et al. (JLab E12-014012 Collaboration), arXiv:1406.4080 [nucl-ex] .

[17] O. Benhar, D. Day, I. Sick, Rev. Mod. Phys. 80 (2008) 189.

[18] O. Benhar, S. C. Pieper, V. R. Pandharipande, Rev. Mod. Phys. 65 (1993) 817.

[19] O. Benhar, Nucl. Phys. News 26 (2016) 15.

[20] C. Marchand, et al., Phys. Rev. Lett 60 (1988) 1703.

[21] D. Rohe, et al (JLab E97-006 Collaboration), Phys. Rev. Lett. 93 (2004) 182501.

[22] D. Rohe (JLab E97-006 Collaboration), Nucl. Phys. B, Proc. Suppl. 159 (2006) 152.

[23] O. Benhar, et al., Phys. Rev. D 72 (2005) 053005.

[24] A. M. Ankowski, O. Benhar, M. Sakuda, Phys. Rev. D 91 (2015) 054616.

[25] R. B. Wiringa, V. G. J. Stoks, R. Schiavilla, Phys. Rev. C 51 (1995) 38.

[26] B.S. Pudliner, et al., Phys. Rev. C 56 (1995) 1720.

[27] E. Epelbaum, H. Hammer, U. Meißner, Rev. Mod. Phys. 81 (2009) 1773.

[28] R. Machleidt, D. R. Entemb, Phys. Rep. 503 (2011) 1.

[29] S. Weinberg, The Quantum Theory of Field, Cambridge University Press, 2005.

[30] D. O. Riska, Phys. Rep. 181 (1989) 207.

[31] J.S. O'Connell, et al, Phys. Rev. C 35 (1987) 1063.

[32] R. Sealock, et al., Phys. Rev. Lett. 62 (1989) 1350.

[33] O. Benhar, J. Phys. Conf. Ser. 408 (2013) 012042.

[34] C. Itzykson, J. Zuber, Quantum Field Theory, McGraw-Hill, New York, 1980.

[35] J. Golak, et al., Phys. Rev. C 52 (1995) 1216.

[36] V. D. Efros, W. Leidemann, G. Orlandini, Phys. Lett. B 338 (1994) 130.

[37] V. D. Efros, W. Leidemann, G. Orlandini, Phys. Rev. Lett. 78 (1997) 432.

[38] V. D. Efros, W. Leidemann, G. Orlandini, Phys. Rev. C 69 (2004) 044001.

[39] J. Carlson, R. Schiavilla, Phys. Rev. Lett. 68 (1992) 3682.

[40] J. Carlson, R. Schiavilla, Rev. Mod. Phys. 70 (1998) 743.

[41] O. Benhar, A. Fabrocini, S. Fantoni, Nucl. Phys. A 505 (1989) 267.

[42] O. Benhar, A. Fabrocini, S. Fantoni, I. Sick, Nucl. Phys. A 579 (1994) $493-517$.

[43] T. de Forest Jr., Nucl. Phys. A392 (1983) 232.

[44] A. E. Dieperink, T. de Forest, I. Sick, R. A. Brandenburg, Phys. Lett. B 63 (1976) 261.

[45] S. Frullani, J. Mougey, Adv. Nucl. Phys. 14 (1984) 1.

[46] A. E. L. Dieperink, P. K. de Witt Huberts, Ann. Rev. Nucl. Part. Sci. 40 (1990) 239.

[47] E.J. Moniz, et al., Phys. Rev. Lett. 26 (1971) 445.

[48] R. Schiavilla, V. Pandharipande, R. Wiringa, Nucl. Phys. A 449 (1986) 219.

[49] O. Benhar, A. Fabrocini, S. Fantoni, Phys. Rev. C 41 (1990) R24(R).

[50] O. Benhar, A. Lovato, N. Rocco, Phys. Rev. C 92 (2015) 024602.

[51] G. B. West, Phys. Rep. 18 (1975) 263.

[52] I. Sick, D. Day, J. S. McCarthy, Phys. Rev. Lett. 45 (1980) 871.

[53] J. Arrington, et al, Phys. Rev. Lett. 82 (????) 2056.

[54] T. W. Donnelly, I. Sick, Phys. Rev. C 60 (1999) 065502.

[55] D. B. Day, et al., Phys. Rev. Lett. 59 (1987) 427.

[56] C. Maieron, et al., Phys. Rev. C 80 (2009) 035504.

[57] J. E. Amaro, M. B. Barbaro, J. A. Caballero, T. W. Donnelly, Phys. Rev. 
Lett. 98 (2007) 242501.

[58] M. C. Martínez, J. A. Caballero, T. W. Donnelly, J. M. Udías, Phys. Rev. Lett. 100 (2008) 052502.

[59] G. Garino, et al, Phys. Rev. C 45 (1992) 780.

[60] T. G. O'Neill, et al, Phys. Lett. B 351 (1995) 87.

[61] D. Abbott, et al, Phys. Rev. Lett. 80 (1998) 5072.

[62] K. Garrow, et al, Phys. Rev. C 66 (2002) 044613.

[63] D. Rohe, et al (JLab E97-006 Collaboration), Phys. Rev. C 72 (2005) 054602.

[64] O. Benhar, Phys. Rev. Lett. 83 (1999) 3130.

[65] S. Boffi, C. Giusti, F. D. Pacati, Phys. Rep. 69 (1992) 881.

[66] J.M. Udías, et al. , Phys. Rev. C 48 (1993) 2731.

[67] A. Meucci, C. Giusti, F. D. Pacati, Phys. Rev. C 64 (2001) 014604.

[68] A. Meucci, Phys. Rev. C 65 (2002) 044601.

[69] C. Giusti, A. Meucci, F. D. Pacati, G. Co', V. De Donno, Phys. Rev. C 84 (2011) 024615.

[70] F. Capuzzi, C. Giusti, F. Pacati, Nucl. Phys. A 524 (1991) 681.

[71] T. R. Sosnik, W. M. Snow, R. N. Silver, P. E. Sokol, Phys. Rev. B 43 (1991) 216.

[72] O. Benhar, et al. , Phys. Rev. C 44 (1991) 2328.

[73] O. Benhar, A. Fabrocini, S. Fantoni, V. R. Pandharipande, I. Sick, Phys. Rev. Lett. 226 (1993) 1.

[74] O. Benhar, Phys. Rev. C 87 (2013) 024606

[75] V. R. Pandharipande, S. C. Pieper, Phys. Rev. C 45 (1992) 791.

[76] O. Benhar, A. Fabrocini, S. Fantoni, V. R. Pandharipande, S. C. Pieper, I. Sick, Phys. Lett. B 359 (1995) 8 - 12.

[77] R. Schiavilla, V. R. Pandharipande, D. O. Riska, Phys. Rev. C 41 (1990) 309.

[78] J. Finn, R. W. Lourie, B. H. Cottmann, Phys. Rev. C 29 (1984) 2230.

[79] P. Barreau, et al., Nucl. Phys. A 402 (1983) 515.

[80] R. Cenni, C. C. degli Atti, G. Salmè, Phys. Rev. C 39 (1989) 1425-1437.

[81] J. Carlson, J. Jourdan, R. Schiavilla, I. Sick, Phys. Rev. C 65 (2002) 024002-.

[82] A. Lovato, S. Gandolfi, J. Carlson, S. C. Pieper, R. Schiavilla, Phys. Rev. Lett. 117 (2016) 082501

[83] A. De Pace, M. Nardi, W. M. Alberico, T. W. Donnelly, A. Molinari, Nucl. Phys. A 726 (2003) $303-326$.

[84] A. Meucci, C. Giusti, F. D. Pacati, Phys. Rev. C 66 (2002) 034610.

[85] O. Benhar, N. Rocco, Adv. High Energy Phys. 2013 (2013) 912702.

[86] O. Benhar, A. Fabrocini, Phys. Rev. C 62 (2000) 034304.

[87] W.M. Alberico, M. Ericson, and A. Molinari, Ann. Phys. 154 (1984) 356

[88] A. Gil, J. Nieves, E. Oset, Nucl. Phys. A 627 (1997) 543 - 598.

[89] S. Cowell, V. R. Pandharipande, Phys. Rev. C 67 (2003) 035504.

[90] O. Benhar, N. Farina, Phys. Lett. B 680 (2009) 305

[91] A. Lovato, C. Losa, O. Benhar, Nucl. Phys. A 901 (2013) $22-50$

[92] A. Lovato, O. Benhar, S. Gandolfi, C. Losa, Phys. Rev. C 89 (2014) 025804.

[93] A. Lovato, S. Gandolfi, J. Carlson, S. C. Pieper, R. Schiavilla, Phys. Rev. C 91 (2015) 062501(R).

[94] A. Lovato, et al., Phys. Rev. Lett. 111 (2013) 092501.

[95] J. Jourdan, Nucl. Phys. A 603 (1996) 117

[96] D. T. Baran, et al., Phys. Rev. Lett. 61 (1988) 400-403.

[97] R. R. Whitney, et al., Phys. Rev. C 9 (1974) 2230-2235.

[98] N. Rocco, A. Lovato, O. Benhar, Phys. Rev. Lett. 116 (2016) 192501.

[99] A. Meucci, J. A. Caballero, C. Giusti, F. D. Pacati, J. M. Udías, Phys. Rev. C 80 (2009) 024605.

[100] R. Gonzaléz-Jimenez, G.D. Megias, M.B. Barbaro, J.A. Caballero, T.W Donnelly, Phys. Rev. C 90 (2014) 035501.

[101] G.D. Megias, J.E. Amaro, M.B. Barbaro, J.A. Caballero, T.W. Donnelly, Phys. Rev. D 94 (2016) 013012.

[102] A. Gil, J. Nieves, E. Oset, Nucl. Phys. A 627 (1997) 599 - 619.

[103] O. Benhar, G. Veneziano, Phys. Lett. B 702 (2011) 433.

[104] O. Benhar, D. Meloni, Nucl. Phys. A 789 (2007) 379-402.

[105] T. Akiri, et al., (LBNE Collaboration), The 2010 Interim Report of the Long-Baseline Neutrino Experiment Collaboration Physics Working Groups .

[106] U. Mosel, O. Lalakulich, K. Gallmeister, Reaction mechanisms at MINERvA, Phys. Rev. D 89 (2014) 093003.

[107] O. Buss, T. Gaitanos, K. Gallmeister, H. van Hees, M. Kaskulov, et al., Transport-theoretical Description of Nuclear Reactions, Phys.Rept. 512
(2012) 1-124.

[108] J. J. Kelly, Phys. Rev. C 70 (2004) 068202.

[109] R. Bradford, A. Bodek, H. Budd, J. Arrington, Nucl. Phys. B Proc. Suppl. 159 (2006) 127.

[110] D. Mund, B. Märkisch, M. Deissenroth, J. Krempel, M. Schumann, H. Abele, A. Petoukhov, T. Soldner, Phys. Rev. Lett. 110 (2013) 172502.

[111] V. Bernard, et al., J. Phys. G 28 (2002) R1.

[112] H. Budd, A. Bodek, J. Arrington, arXiv:hep-ex/0308005

[113] O. Lalakulich, E. A. Paschos, Phys. Rev. D 71 (2005) 074003.

[114] M. S. Athar, S. Chauhan, S. K. Singh, Eur. Phys. J. A 43 (2010) 209.

[115] T. Leitner, O. Buss, L. Alvarez-Ruso, U. Mosel, Electron- and neutrinonucleus scattering from the quasielastic to the resonance region, Phys. Rev. C 79 (2009) 034601.

[116] P. Berge, et al., Zeit. Phys. C 49 (1991) 187

[117] R. G. Roberts, The Structure of the Proton, Cambridge University Press, Cambridge, 1990.

[118] M. Glück, E. Reya, A. Vogt, Eur. Phys. J. C 5 (1998) 461.

[119] S. A. Kulagin, R. Petti, Phys. Rev. D 76 (2007) 094023.

[120] H. Haider, I. R. Simo, M. S. Athar, M. J. V. Vacas, Phys. Rev. C 84 (2011) 054610

[121] M. Hirai, S. Kumano, M. Miyama, Phys. Rev. D 64 (2001) 034003.

[122] R. Gran, et al., (K2K Collaboration), Phys. Rev. D 74 (2006) 052002.

[123] V. Lyubushkin et al. (NOMAD Collaboration), Eur. Phys. J. C 63 (2009) 355-381.

[124] O. Benhar, P. Coletti, D. Meloni, Phys. Rev. Lett. 105 (2010) 132301.

[125] M. Martini, M. Ericson, G. Chanfray, Phys. Rev. C 84 (2011) 055502.

[126] J. Nieves, I. Ruiz Simo, M. Vicente Vacas, Phys. Lett. B 707 (2012) $72-75$.

[127] R. Gran, J. Nieves, F. Sanchez, M. Vicente Vacas, Phys. Rev. D 88 (2013) 113007

[128] J. Marteau, J. Delorme, M. Ericson, Nucl. Phys. A 663 \& 664 (2000) $783 \mathrm{c}$.

[129] J. Delorme, P. A. M. Guichon, Phys. Lett. B 293 (1991) 157.

[130] J. Marteau, Eur. Phys. J. A 5 (1999) 183.

[131] G.D. Megias, et al., Phys. Rev. D 94 (2016) 093004.

[132] A. M. Ankowski, J. T. Sobczyk, Phys. Rev. C 77 (2008) 044311.

[133] C. Andreopoulos, A. Bell, D. Bhattacharya, F. Cavanna, J. Dobson, et al., Nucl. Instrum. Meth. A614 (2010) 87-104.

[134] C. M. Jen, A. Ankowski, O. Benhar, A. P. Furmanski, L. N. Kalousis, et al., Phys. Rev. D 90 (2014) 093004.

[135] T. Golan, C. Juszczak, J. T. Sobczyk, Phys. Rev. C 86 (2012) 015505.

[136] K. Abe, et al. (T2K Collaboration), Phys. Rev. D 91 (2015) 112002.

[137] S. Dytman, AIP Conf. Proc. 1382 (2011) 156-157.

[138] S. A. Dytman, A. S. Meyer, AIP Conference Proceedings 1405 (2011) 213-218.

[139] C. Williamson, et al., Phys. Rev. C 56 (1997) 3152-3172.

[140] M. Anghinolfi, M. Ripani, R. Cenni, P. Corvisiero, A. Longhi, L. Mazzaschi, V. Mokeev, G. Ricco, M. Taiuti, A. Teglia, A. Zucchiatti, N. Bianchi, A. Fantoni, V. Muccifora, P. LeviSandri, V. Lucherini, E. Polli, A. R. Reolon, P. Rossi, S. Simula, J. Phys. G 21 (1997) L9.

[141] K.N. Abazajian, et al., arXiv:1204.5379 [hep-ph]

[142] P. Adamson, et al. (MINOS Collaboration), Phys. Rev. D 81 (2010) 072002.

[143] H. Higuera, et al. (MINER $v$ A Collaboration), arXiv:1409.3835 [hep-ex]

[144] S. Choubey, et al. (IDS-NF Collaboration), arXiv:1112.2853 [hep-ex]

[145] D. Adey, et al.al. (vSTORM Collaboration), arXiv:1308.6822 [physics.acc-ph]

[146] M. Freund, Phys. Rev. D 64 (2001) 053003.

[147] S. Antusch, P. Huber, S. King, T. Schwetz, JHEP 0704 (2007) 060.

[148] R. D. Peccei, Lect. Notes Phys. 741 (2008) 3.

[149] S. Ritz, et al.al. (HEPAP Subcommittee), Building for Discovery: Strategic Plan for U.S. Particle Physics in the Global Context, http://science.energy.gov/ /media/hep/hepap/pdf/ May2014/FINAL_P5_Report_053014.pdf

[150] M. Gonzalez-Garcia, M. Maltoni, J. Salvado, T. Schwetz, JHEP 1212 (2012) 123.

[151] M. Day, K. S. McFarland, Phys. Rev. D 86 (2012) 053003.

[152] D.G. Michael, et al. (MINOS COllaboration), Phys. Rev. Lett. 97 (2006) 191801.

[153] K. Abe, et al. (T2K Collaboration), Phys. Rev. Lett. 113 (2014) 241803. 
[154] P. Huber, M. Mezzetto, T. Schwetz, JHEP 0803 (2008) 021.

[155] P. Coloma, P. Huber, J. Kopp, W. Winter, Phys. Rev. D 87 (2013) 033004.

[156] P. Zucchelli, Phys. Lett. B 532 (2002) 166.

[157] E. Fernandez-Martinez, D. Meloni, Phys. Lett. B 697 (????) 477.

[158] D. Meloni, M. Martini, Phys. Lett. B 716 (2012) 186.

[159] Y. Hayato, Nucl. Phys. B Proc. Suppl. 112 (2002) 171.

[160] A. Rodriguez, et al. (K2K Collaboration), Phys. Rev. D 78 (2008) 032003.

[161] A.A. Aguilar-Arevalo, et al. (MiniBooNE Collaboration, Phys. Rev. Lett. 103 (2009) 081801.

[162] Y. Kurimoto, et al. (SciBooNE Collaboration), Phys. Rev. D 81 (2010) 033004.

[163] Y. Nakajima, et al. (SciBooNE Collaboration), Phys. Rev. D 83 (2011) 012005.

[164] R. A. Smith, E. J. Moniz, Nucl. Phys. B 43 (1972) 605.

[165] D. Rein, L. M. Sehgal, Ann. Phys. 133 (1981) 79.

[166] M. Martini, M. Ericson, G. Chanfray, J. Marteau, Phys. Rev. C 80 (2009) 065501.

[167] P. Huber, M. Lindner, W. Winter, Comput. Phys. Commun. 167 (2005) 195.

[168] P. Huber, J. Kopp, M. Lindner, M. Rolinec, W. Winter, Comput. Phys. Commun. 177 (2007) 432.

[169] M. Blennow, E. Fernandez-Martinez, Comput. Phys. Commun. 181 (2010) 227-231.

[170] K. Abe, et al. (T2K Collaboration), Phys. Rev. Lett. 107 (2011) 041801.

[171] K. Abe, et al. (T2K Collaboration), Phys. Rev. D 85 (2012) 031103.

[172] A. Cervera, et al., Nucl. Phys. b 579 (2000) 17.

[173] A. Donini, E. Fernandez-Martinez, D. Meloni, S. Rigolin, Nucl. Phys. B 743 (2006) 41.

[174] P. Coloma, P. Huber, C.-M. Jen, C. Mariani, Phys. Rev. D 89 (2014) 073015.

[175] O. Lalakulich, U. Mosel, K. Gallmeister, Phys. Rev. C 86 (2012) 054606.

[176] M. Martini, M. Ericson, G. Chanfray, Phys. Rev. D 85 (2012) 093012.

[177] J. Nieves, F. Sanchez, I. Ruiz Simo, M. Vicente Vacas, Phys. Rev. D 85 (2012) 113008.

[178] U. Mosel, O. Lalakulich, K. Gallmeister, Phys. Rev. Lett. 112 (2014) 151802 .

[179] P. Coloma, P. Huber, Phys. Rev. Lett. 111 (2013) 221802.

[180] A. M. e. a. Ankowski, Comparison of the calorimetric and kinematic methods of neutrino energy reconstruction in disappearance experiments, Phys. Rev. D92 (7) (2015) 073014.

[181] A. M. e. a. Ankowski, Missing energy and the measurement of the CP-violating phase in neutrino oscillations, Phys. Rev. D92 (9) (2015) 091301.

[182] L. A. et al, Design, calibration, and performance of the $\{$ MINERvA detector, Nuclear Instruments and Methods in Physics Research Section A: Accelerators, Spectrometers, Detectors and Associated Equipment 743 (2014) 130 - 159, ISSN 0168-9002.

[183] P. Huber, M. Lindner, T. Schwetz, W. Winter, First hint for CP violation in neutrino oscillations from upcoming superbeam and reactor experiments, JHEP 0911 (2009) 044.

[184] R. Acciarri, et al., Long-Baseline Neutrino Facility (LBNF) and Deep Underground Neutrino Experiment (DUNE) .

[185] Q. Wu, et al. (NOMAD Collaboration), Phys. Lett. B 660 (2008) 19.

[186] K. Abe, et al. (T2K Collaboration), Phys. Rev. D 92 (2015) 112003.

[187] Y. Nakajima, et al. (SciBooNE Collaboration), Phys. Rev. D 83 (2011) 012005.

[188] J. Carlson, V. R. Pandharipande, R. Schiavilla, Phys. Rev. C 47 (1993) 484.

[189] O. Benhar, JPS Conf. Proc. 12 (2016) 010001. 